\title{
Revisiting the characteristic initial value problem for the vacuum Einstein field equations
}

\author{
David Hilditch ${ }^{1}$. Juan A. Valiente Kroon ${ }^{2}$ (D) Peng Zhao ${ }^{2}$
}

Received: 28 April 2020 / Accepted: 16 September 2020 / Published online: 6 October 2020

(c) The Author(s) 2020

\begin{abstract}
Using the Newman-Penrose formalism we study the characteristic initial value problem in vacuum General Relativity. We work in a gauge suggested by Stewart, and following the strategy taken in the work of Luk, demonstrate local existence of solutions in a neighbourhood of the set on which data are given. These data are given on intersecting null hypersurfaces. Existence near their intersection is achieved by combining the observation that the field equations are symmetric hyperbolic in this gauge with the results of Rendall. To obtain existence all the way along the null-hypersurfaces themselves, a bootstrap argument involving the Newman-Penrose variables is performed.
\end{abstract}

Keywords Characteristic problem · Newman-Penrose formalism · Initial value problem

\section{Contents}

1 Introduction ................................ 2

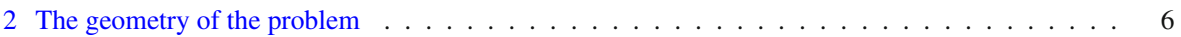

3 The initial data for the CIVP . . . . . . . . . . . . . . . . . . . . . . . 11

4 Rendall's local existence theory . . . . . . . . . . . . . . . . . . . . . . . . . . . 14

5 Setting-up Luk's strategy . . . . . . . . . . . . . . . . . . . . . . . . . . . . . . . . . . . . 19

6 Main estimates . . . . . . . . . . . . . . . . . . . . . . . . . . . . 34

7 Last slice argument and the end of the proof . . . . . . . . . . . . . . . . . . . 60

$\bowtie \quad$ Juan A. Valiente Kroon

j.a.valiente-kroon@qmul.ac.uk

David Hilditch

david.hilditch@tecnico.ulisboa.pt

Peng Zhao

p.zhao@qmul.ac.uk

1 CENTRA, Departamento de Física, Instituto Superior Técnico - IST, Universidade de Lisboa UL, Avenida Rovisco Pais 1, 1049 Lisbon, Portugal

2 School of Mathematical Sciences, Queen Mary, University of London, Mile End Road, London E1 4NS, UK 
A: The Einstein field equations in the NP formalism . . . . . . . . . . . . . . . . . . . . . 64

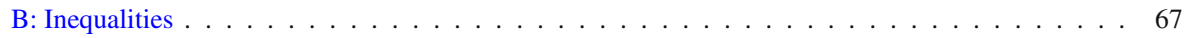

C: Angular derivatives of a scalar function . . . . . . . . . . . . . . . . . . . . . 68

D: Integration Identities . . . . . . . . . . . . . . . . . . . . . . . . . . . . . 71

E: Details in Propositions 8 and $9 \ldots \ldots \ldots \ldots \ldots \ldots \ldots \ldots$

References . . . . . . . . . . . . . . . . . . . . . . 75

\section{Introduction}

The simplest setups of partial differential equations (PDEs) are of course the boundary value and Cauchy/initial value problems (IVPs). For hyperbolic PDEs the IVP is of particular interest since it naturally forms a well-posed problem. Rather than specifying data just on a spacelike hypersurface as in the IVP however, we can consider additionally the initial boundary value problem. In this setup might have, for example, a compact spatial domain and then choose suitable boundary conditions on a timelike worldtube at the perimeter of that domain. A third possibility, that we consider in the present work, is the characteristic initial value problem (CIVP). Here data are specified on characteristic surfaces of the equations under consideration. In the context of general relativity (GR) these surfaces are null slices.

In GR the CIVP has a long history which dates back at least to the pioneering work by Bondi and collaborators on gravitational waves-see [1,2]. The analysis in this work is based on the observation that in coordinates (Bondi coordinates) adapted to the geometry of outgoing light cones, the Einstein equations give rise to a hierarchy of equations which can be formally solved in sequence if certain pieces of data are provided. These ideas were formalised in subsequent work by Sachs-see [3]. The CIVP was reconsidered by Newman \& Penrose in their more geometric reformulation of the original analysis of gravitational radiation by Bondi and collaborators-see [4], which also contains the original formulation of the frame formulation of the Einstein field equations known as the Newman-Penrose (NP) formalism. The work by Newman $\&$ Penrose identifies particular components of the Weyl tensor (expressed in terms of a null frame) as the key pieces of free data to be specified on the characteristic hypersurfaces. The CIVP setup also underlies subsequent work by Penrose on the properties of massless spin fields and his approach of exact sequences of fieldssee [5]. The common theme in this early work on the CIVP in GR is that is mainly concerned with the structural (i.e. algebraic) properties of the system of equations and does not systematically address the issue of existence and uniqueness of solutions.

Pioneering work on technical issues concerning the existence and uniqueness of solutions to the characteristic problem for the Einstein field equations can be found in the analysis of Müller zu Hagen and Seifert [6]. These ideas were brought to fruition in the work of Friedrich-see [7]. There, it was shown that the formulation of the characteristic problem by Newman \& Penrose implies a symmetric hyperbolic evolution system for which known techniques from the theory of PDEs can be applied. In particular, Friedrich shows the local existence of solutions near the intersection of the characteristic hypersurfaces under the assumption of analyticity of the freely specifiable data. This method was extended in subsequent work to characteristic problems for a conformal representation of the Einstein field equations (the the conformal 
Einstein field equations)—see [8,9]. Among other things, this work demonstrates the mathematical consistency of the work on the nature of gravitational waves by Bondi and collaborators and Newman \& Penrose. The formulation of the CIVP for the Einstein equations using the NP formalism was further developed as a possible pathway towards numerical simulations of the Einstein field equations [10] — see also [11] for an alternative formulation for numerics using the Bondi approach to the characteristic problem, and also influenced work on the nature and classification of caustics in Relativity [12].

A major milestone in the analysis of the problem came with the influential work by Rendall on the reduction of the CIVP to a standard IVP [13], whose well-posedness is guaranteed by the classical results of Choquet-Bruhat [14]. In particular this reduction provides a local existence theorem for the CIVP for the Einstein field equations which only requires a finite level of differentiability of the initial data. Rendall's method was subsequently used to obtain a smooth data version Friedrich local existence result for the asymptotic CIVP for the conformal Einstein field equations. Ideas arising from the CIVP underline and permeate the fundamental work by Christodoulou \& Klainermann and on the non-linear stability for the Einstein field equations [15,16]. In particular, Christodoulou \& Klainermann make use of a null frame formalism related to that of Newman \& Penrose. Moreover, their analysis systematically exploits the nonlinear structure of the Einstein field equations when expressed in terms of such a null frame.

The structural properties identified in the analysis by Christodoulou \& Klainermann paved the way for an improved local existence result for the CIVP for the Einstein equations. Working in a gauge adopted from Christodoulou's work on the formation of black holes [17], which explicitly employs double-null coordinates, such an improved result has been given by Luk [18]. This work guarantees an existence domain no longer restricted to a neighbourhood of the intersection of the initial null hypersurfaces but that stretches along them. Recently, Luk's analysis has been extended so that the existence interval extends arbitrarily along the null hypersurfaces and, thus, the solution contains a piece of infinity-see [19]. An alternative approach to an improve local existence result for the CIVP has been pursued by Chruściel and collaborators-see [20-22] This approach makes use of second order evolution equations for which well developed theory of the CIVP exists-see e.g. [23,24].

Presently we are interested in two follow-up questions for which the work of Rendall [13] and Luk [18] are most relevant. Firstly, how do the aforementioned results look when expressed in the language of the Newman-Penrose formalism? Following long-term existence results in harmonic gauge [25], it is apparent that a variety of formulations of GR exhibit desirable structure in their nonlinearities. Second, we are therefore curious as to the robustness of this 'null-structure' under changes of gauge. We hence give a formulation of the CIVP heavily influenced by that of Stewart [26], and demonstrate for that formulation local existence in a full neighbourhood of the initial null surfaces. In first instance, the argument here provided gives an improved local existence result along one of the initial hypersurfaces. This argument can be adapted, mutatis mutandi, to obtain improved local existence along the other initial hypersurface-see Fig. 1b. For conciseness, we restrict our discussion to the neighbourhood of only one of the hypersurfaces. The precise statement of our main result is given in Theorem 4 in Sect. 7.3. 
A tertiary aim in a reformulation in terms of the NP formalism is to allow for the arguments and methods employed in Christodoulou's formulation to be recast for application elsewhere. Although the NP formalism has played an influential role in mathematical Relativity, for historical reasons, its use in the formulation of existence results has been limited. Our interest in understanding the structural properties of the NP field equations is what drives us to consider the approach to an improved local existence result for the CIVP pursued by Luk rather than the one followed by Chruściel and collaborators. In the future we hope that this will permit us to obtain similar results for the conformal field equations [8,9]. Regarding the question of robustness of the nonlinearities, our work serves only as a stepping stone for a more detailed investigation. Nevertheless it is worth stressing that our gauge differs from that used elsewhere, and that the nonlinearities of the equations do retain sufficient structure for us to successfully follow through the argument (Fig. 2).

Remark 1 Throughout this article the phrase improved existence result is used in the sense of an improvement on Rendall's existence result in a neighbourhood of the intersection of the initial null hypersurfaces. The improved results are, in a sense, optimal in that they provide existence in a neighbourhood of the initial hypersurfaces as long as one has control on the initial data—see Fig. 1.

\section{New insights}

Although the main result of our analysis, stated in Theorem 4, is in geometric terms essentially equivalent to the improved existence result by Luk in [18] our analysis provides, in addition, a wealth of insight into the structural properties of the NP equations. In order to attain this aim we feel that it is necessary to give a detailed discussion of of the various arguments to convince the reader that they indeed follow through. Moreover, we also provide details on a number of technical results which cannot be found elsewhere in the literature - these include: (i) a detailed identification of the reduced initial data for the characteristic initial value problem; (ii) the verification that Stewart's gauge does indeed leads to a symmetric hyperbolic evolution system; (iii) a verification that Rendall's strategy for the reduction of the characteristic initial value problem to a standard initial value problem can be applied to the NP equations in Stewart's gauge (in order to do this additional structural properties beyond symmetric hyperbolicity are required). Moreover, (iv) while Luk's analysis requires the use of a certain type of elliptic estimates to control tensor fields over topological 2-spheres, our approach based on the use of scalar quantities does not require this type of arguments. This makes our proof technically simpler. Finally, (v) we provide a detailed discussion of the inner workings of last slice argument used to obtain the main existence result.

\section{Outline of the article}

In Sect. 2 we provide a formulation of the propblem under consideration and discuss the technical details involved in the construction of the choice of gauge for the Einstein field equations (Stewart's gauge). Section 3 provides a discussion of how to prescribe 
(a)

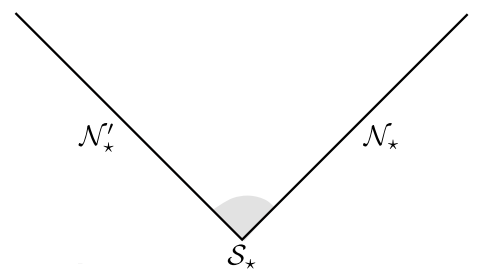

(b)

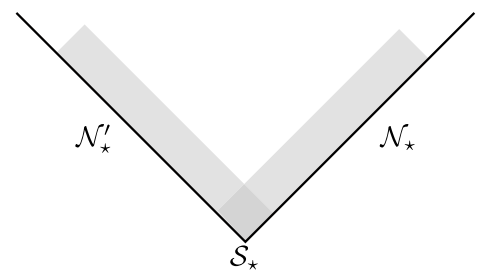

Fig. 1 Comparison of the existence domains for the characteristic problem: a existence domain using Rendall's strategy based on the reduction to a standard Cauchy problem; b existence domain using Luk's strategy -in principle, the long side of the rectangles extends for as much as one has control on the initial data

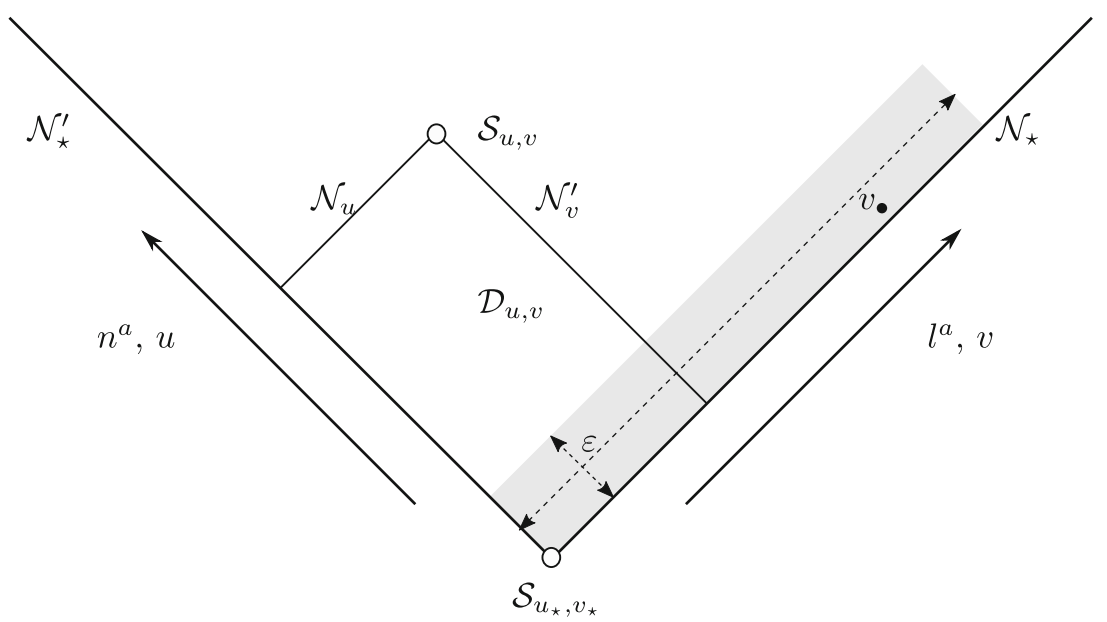

Fig. 2 Setup for Stewart's gauge. The construction makes use of a double null foliation of the future domain of dependence of the initial hypersurface $\mathcal{N}_{\star} \cup \mathcal{N}_{\star}$. The coordinates and NP null tetrad are adapted to this geometric setting. The analysis in this article is focused on the arbitrarily thin grey rectangular domain along the hypersurface $\mathcal{N}_{\star}$. The argument can be adapted, in a suitable manner, to a similar rectangle along $\mathcal{N}_{\star}$. See the main text for the definitions of the various regions and objects

initial data for the evolution equations. Section 4 provides a formulation of the local existence result for the characteristic initial value problem using Rendall's reduction strategy. The latter includes an analysis of the symmetric hyperbolicity of the evolution equations, the computation of formal derivatives on the initial hypersurfaces, the propagation of the constraints and a brief overview of Rendall's method. Section 5 contains a discussion of the basic setup for Luk's method. This section includes, in particular, an overview of the steps in the argument, a discussion of the analytical tools which includes the construction of various $L^{p}$-type estimates for transport equations and Sobolev inequalities. Section 6 provides a detailed discussion of the construction of the main estimates required in the improved existence result. Section 7 contains a discussion of the last slice argument and the statement of the main result in Theorem 4. The article has five appendices. "Appendix A" list the Einstein equations in NP formulation used in the main text. "Appendix B" provides a quick-reference list of the main 
inequalities used in our analysis. "Appendix C" provides further detail on the computation of angular derivatives of scalar fields. "Appendix D" provides some further details on some integral identities used in the main text. Finally, "Appendix E" gives extended detail on the computations of some of the key propositions in our analysis.

\section{Notation and conventions}

We take $\{a, b, c, \ldots\}$ to denote abstract tensor indices whereas $\{\mu, v, \lambda, \ldots\}$ will be used as spacetime coordinate indices with the values $0, \ldots, 3$. Our conventions for the curvature tensors are fixed by the relation

$$
\left(\nabla_{a} \nabla_{b}-\nabla_{b} \nabla_{a}\right) v^{c}=R_{d a b}^{c} v^{d}
$$

We make systematic use of the NP formalism as described, for example, in [26,27]. In particular, the signature of Lorentzian metrics is $(+---)$. Many of our derivations, although straightforward, are fairly lengthy, so we have included in "Appendix A" a complete summary of the equations of the NP-formalism, highlighting the simplifications that occur with our particular gauge. We recommend that the reader keep a copy of the appendix to hand as they read the paper.

\section{The geometry of the problem}

Let $(\mathcal{M}, \boldsymbol{g})$ denote a vacuum spacetime satisfying $R_{a b}=0$, where $\mathcal{M}$ is a 4 dimensional manifold with boundary and an edge. The boundary consists of two null hypersurface: $\mathcal{N}_{\star}$, the outgoing null hypersurface; $\mathcal{N}_{\star}$, the incoming null hypersurface with non-empty intersection $\mathcal{S}_{\star} \equiv \mathcal{N}_{\star} \cap \mathcal{N}_{\star}$. We will assume that $\mathcal{S}_{\star} \approx \mathbb{S}^{2}$. Given a neighbourhood $\mathcal{U}$ of $\mathcal{S}_{\star}$, one can introduce coordinates $x=\left(x^{\mu}\right)$ with $x^{0}=v$ and $x^{1}=u$ such that, at least in a neighbourhood of $\mathcal{S}_{\star}$ one can write

$$
\mathcal{N}_{\star}=\{p \in \mathcal{U} \mid u(p)=0\}, \quad \mathcal{N}_{\star}=\{p \in \mathcal{U} \mid v(p)=0\}
$$

Given suitable data on $\left(\mathcal{N}_{\star} \cup \mathcal{N}_{\star}\right) \cap \mathcal{U}$ we are interested in making statements about the existence and uniqueness of solutions to the vacuum Einstein field equations of the aforementioned type on some open set

$$
\mathcal{V} \subset\{p \in \mathcal{U} \mid u(p) \geq 0, v(p) \geq 0\}
$$

which we identify with a subset of the future domain of dependence, $D^{+}\left(\mathcal{N}_{\star} \cup \mathcal{N}_{\star}\right)$, of $\mathcal{N}_{\star} \cup \mathcal{N}_{\star}$.

\subsection{Construction of the gauge: Stewart's approach}

We will ultimately be concerned with existence and uniqueness of solutions, but, as is common in such constructions, it is useful to start by assuming existence in order to 
give a concrete PDE formulation of the problem. In this section we thus briefly review the gauge choice. In the rest of this article we will call this construction Stewart's gauge-see e.g. [26], Section 4.3.

\subsubsection{Coordinates}

In the following it will be convenient to regard the 2-dimensional surface $\mathcal{S}_{\star}$ as a submanifold of a spacelike hypersurface $S$. The subsequent discussion will be restricted to the future of $S$. The hypersurface $S$ is an auxiliary structure which, although it does not belong to $D^{+}\left(\mathcal{N}_{\star} \cup \mathcal{N}_{\star}\right)$, plays a key role in Rendall's strategy to reduce the characteristic initial value problem to a standard initial value problem-see Sect. 4.2, below. As $\mathcal{S}_{\star} \approx \mathbb{S}^{2}$, one has that $\mathcal{S}_{\star}$ divides $S$ in two regions-the interior of $\mathcal{S}_{\star}$ and the exterior of $\mathcal{S}_{\star}$. Now, consider a foliation of $S$ by 2-dimensional surfaces with the topology of $\mathbb{S}^{2}$ which includes $\mathcal{S}_{\star}$. At each of the 2-dimensional surfaces we assume there pass two null hypersurfaces. Further, we assume that:

(i) One of these hypersurfaces has the property that the projection of the tangent vectors of their generators at $\mathcal{S}_{\star}$ point outwards -we call these null hypersurfaces outgoing light cones;

(ii) One of these hypersurfaces has the property that the projection of the tangent vectors of their generators at $\mathcal{S}_{\star}$ point inwards-we call these null hypersurfaces ingoing light cones.

Thus, as least close to $S$ one obtains a 1-parameter family of outgoing null hypersurfaces $\mathcal{N}_{u}$ and a 1-parameter family of ingoing null hypersurfaces $\mathcal{N}_{v}$. One can then define scalar fields $u$ and $v$ by the requirements, respectively, that $u$ is constant on each of the $\mathcal{N}_{u}$ and $v$ is constant on each $\mathcal{N}_{v}$. In particular, we assume that $\mathcal{N}_{0}=\mathcal{N}_{\star}$ and $\mathcal{N}_{0}=\mathcal{N}_{\star}$. Following standard usage, we call $u$ a retarded time and $v$ an advanced time. We use the notation $\mathcal{N}_{u}\left(v_{1}, v_{2}\right)$ to denote the part of the hypersurface $\mathcal{N}_{u}$ with $v_{1} \leq v \leq v_{2}$. Likewise $\mathcal{N}_{v}\left(u_{1}, u_{2}\right)$ has a similar definition. We denote the sphere intersected by $\mathcal{N}_{u}$ and $\mathcal{N}_{v}$ by $\mathcal{S}_{u, v}$. We define the region

$$
\bigcup_{0 \leq v^{\prime} \leq v, 0 \leq u^{\prime} \leq u} \mathcal{S}_{u^{\prime}, v^{\prime}}
$$

as $\mathcal{D}_{u, v}$. We also define the time function

$$
t \equiv u+v,
$$

and the truncated causal diamond,

$$
\mathcal{D}_{u, v}^{\tilde{t}} \equiv \mathcal{D}_{u, v} \cap\{t \leq \tilde{t}\},
$$

which will be used frequently throughout our arguments.

The scalar fields $u$ and $v$ introduced in the previous paragraph will be used as coordinates in a neighbourhood of $\mathcal{S}_{\star}$. To complete the coordinate system, consider arbitrary coordinates $\left(x^{\mathcal{A}}\right)$ on $\mathcal{S}_{\star}$, with the index $\mathcal{A}$ taking the values 2 , 3. These 
coordinates are then propagated into $\mathcal{N}_{\star}$ by requiring them to be constant along the generators of $\mathcal{N}_{\star}$. Once coordinates have been defined on $\mathcal{N}_{\star}$, one can propagate them into $\mathcal{V}$ by requiring them to be constant along the generators of each $\mathcal{N}_{v}$. In this manner one obtains a coordinate system $\left(x^{\mu}\right)=\left(v, u, x^{\mathcal{A}}\right)$ in $\mathcal{V}$.

\subsubsection{The NP frame}

To construct a null NP tetrad we choose vector fields $l^{a}$ and $n^{a}$ to be tangent to the generators of $\mathcal{N}_{u}$ and $\mathcal{N}_{v}$ respectively. Further we require them to be normalised according to

$$
g_{a b} l^{a} n^{b}=1
$$

The latter normalisation condition is preserved under the boost,

$$
l^{a} \mapsto \varsigma l^{a}, \quad n^{a} \mapsto \varsigma^{-1} n^{a}, \quad \varsigma \in \mathbb{R} .
$$

This freedom can be used to set

$$
n_{a}=\nabla_{a} v
$$

This requirement still leaves some freedom left as one can choose a relabelling of the form $v \mapsto V(v)$. Next, we choose the complex vector fields $m^{a}$ and $\bar{m}^{a}$ so that they are tangent to the surfaces $\mathcal{S}_{u, v}$ and satisfy the conditions

$$
g_{a b} m^{a} \bar{m}^{b}=-1, \quad g_{a b} m^{a} m^{b}=0 .
$$

There is still the freedom to perform a spin

$$
m^{a} \mapsto e^{i \theta} m^{a}, \quad \theta \in \mathbb{R}
$$

at each point.

Remark 2 It can be verified that the vectors $\left\{l^{a}, n^{a}, m^{a}, \bar{m}^{a}\right\}$ constructed in the previous paragraphs satisfy

$$
g_{a b} l^{a} m^{b}=g_{a b} n^{a} m^{b}=g_{a b} l^{a} \bar{m}^{b}=g_{a b} n^{a} \bar{m}^{b}=0 .
$$

Now, observing that, by construction, on the generators of each null hypersurface $\mathcal{N}_{v}^{*}$ only the coordinate $u$ varies, one has that

$$
n^{\mu} \boldsymbol{\partial}_{\mu}=Q \partial_{u}
$$

where $Q$ is a real function of the position. Furthermore, since the vector $l^{a}$ is tangent to the generators of each $\mathcal{N}_{u}$ and $l^{a} n_{a}=l^{a} \nabla_{a} v=1$, one has that

$$
l^{\mu} \boldsymbol{\partial}_{\mu}=\boldsymbol{\partial}_{v}+C^{\mathcal{A}} \boldsymbol{\partial}_{\mathcal{A}},
$$


where, again, the components $C^{\mathcal{A}}$ are real functions of the position. By construction, the coordinates $\left(x^{\mathcal{A}}\right)$ do not vary along the generators of $\mathcal{N}_{\star}$-that is, one has that $l^{a} \nabla_{a} x^{\mathcal{A}}=0$. Accordingly, one has that

$$
C^{\mathcal{A}}=0 \text { on } \mathcal{N}_{\star} .
$$

Finally, since $m^{a}$ and $\bar{m}^{a}$ span the tangent space of each surface $\mathcal{S}_{u, v}$ one has that

$$
m^{\mu} \boldsymbol{\partial}_{\mu}=P^{\mathcal{A}} \boldsymbol{\partial}_{\mathcal{A}}
$$

where the coefficients $P^{\mathcal{A}}$ are complex functions.

Summarising, we make the following choice:

Gauge choice 1 (Stewart's choice of the components of the frame) On $\mathcal{V}$ we consider a Newman-Penrose frame $\left\{l^{a}, n^{a}, m^{a}, \bar{m}^{a}\right\}$ of the form:

$$
\boldsymbol{l}=\boldsymbol{\partial}_{v}+C^{\mathcal{A}} \boldsymbol{\partial}_{\mathcal{A}}, \quad \boldsymbol{n}=Q \boldsymbol{\partial}_{u}, \quad \boldsymbol{m}=P^{\mathcal{A}} \boldsymbol{\partial}_{\mathcal{A}} .
$$

Remark 3 In view of the normalisation condition $g_{a b} m^{a} \bar{m}^{b}=-1$, there are only 3 real functions involved in the $P^{\mathcal{A}}$ 's. Thus, $Q, C^{\mathcal{A}}$ together with $P^{\mathcal{A}}$ give six scalar fields describing the metric. Thus the components $\left(g^{\mu \nu}\right)$ of the contravariant form of the metric $g$ are of the form

$$
\left(g^{\mu \nu}\right)=\left(\begin{array}{ccc}
0 & Q & 0 \\
Q & 0 & Q C^{\mathcal{A}} \\
0 & Q C^{\mathcal{A}} & \sigma^{\mathcal{A B}}
\end{array}\right)
$$

where

$$
\sigma^{\mathcal{A B}} \equiv-\left(P^{\mathcal{A}} \overline{P^{\mathcal{B}}}+\overline{P^{\mathcal{A}}} P^{\mathcal{B}}\right)
$$

Here and in what follows $\sigma$ is the induced metric on $\mathcal{S}_{u, v}$, and has contravariant components $\sigma^{\mathcal{A B}}$ defined in the standard manner. Note that care is needed to distinguish $\sigma$, the NP connection coefficient, from this quantity. From the expression, we can compute that $l_{\mu} d x^{\mu}=Q^{-1} d u, \sigma_{\mathcal{A B}} P^{\mathcal{A}} P^{\mathcal{B}}=0, \sigma_{\mathcal{A B}} P^{\mathcal{A}} P^{\mathcal{B}}=-1$ and $-\partial_{\mathcal{A}} C^{\mathcal{A}}=\bar{m}_{\mathcal{A}} \delta C^{\mathcal{A}}+m_{\mathcal{A}} \bar{\delta} C^{\mathcal{A}}$ directly.

Remark 4 On $\mathcal{N}_{\star}$ one has that $\boldsymbol{n}=Q \boldsymbol{\partial}_{u}$. As the coordinates $\left(x^{\mathcal{A}}\right)$ are constant along the generators of $\mathcal{N}_{\star}$ and $\mathcal{N}_{\star}$, it follows that on $\mathcal{N}_{\star}$ the coefficient $Q$ is only a function of $\mathrm{u}$. Thus, without loss of generality one can parameterise $u$ so as to set $Q=1$ on $\mathcal{N}_{\star}$.

\subsection{Analysis of the NP commutators}

In this subsection we analyse some simple consequences of the NP frame of Gauge choice 1 and the NP commutator Eqs. (29a)-(29d). In particular, we exploit the fact 
that given a choice of NP frame, the evaluation of the NP commutators on the coordinates gives rise to two different types of equations, namely (i) conditions on the spin connection coefficients, and (ii) equations for the coefficients of the frame. In what follows we analyse these two classes of equations. For future use observe that from the definition of the NP frame $\left\{l^{a}, n^{a}, m^{a}, \bar{m}^{a}\right\}$ in the gauge choice 1 it readily follows that,

$$
\begin{aligned}
& D v=1, \quad \Delta v=0, \quad \delta v=0, \quad \bar{\delta} v=0, \\
& D u=0, \quad \Delta u=Q, \quad \delta u=0, \quad \bar{\delta} u=0, \\
& D x^{\mathcal{A}}=C^{\mathcal{A}}, \quad \Delta x^{\mathcal{A}}=0, \quad \delta x^{\mathcal{A}}=P^{\mathcal{A}}, \quad \bar{\delta} x^{\mathcal{A}}=\bar{P}^{\mathcal{A}} \text {. }
\end{aligned}
$$

\subsubsection{Spin connection coefficients}

Direct inspection of the NP commutators (29a)-(29d) applied to the coordinates $\left(v, u, x^{2}, x^{3}\right)$ taking into account $(9 \mathrm{a})-(9 \mathrm{c})$ yields on $\mathcal{V}$ the conditions,

$$
\kappa=v=0, \quad \gamma+\bar{\gamma}=0, \quad \rho=\bar{\rho}, \quad \mu=\bar{\mu}, \quad \pi=\alpha+\bar{\beta} .
$$

We will see that these gauge conditions can be refined still further.

Fixing the rotation freedom The set up of frame vectors under the frame choice 1 allows the freedom of a rotation

$$
m^{a} \mapsto m^{\prime a}=e^{i \theta} m^{a} .
$$

The latter, in turn, implies the transformation

$$
\gamma-\bar{\gamma} \mapsto \gamma^{\prime}-\bar{\gamma}^{\prime}=\gamma-\bar{\gamma}-i \Delta \theta
$$

Accordingly, by requiring $\theta$ to satisfy the equation

$$
\Delta \theta=i(\bar{\gamma}-\gamma)
$$

it is always possible to assume that $\bar{\gamma}-\gamma=0$, which, together with the condition $\gamma+$ $\bar{\gamma}=0$ allows us to set $\gamma=0$ on $\mathcal{V}$. A similar computation shows that

$$
\epsilon-\bar{\epsilon} \mapsto \epsilon^{\prime}-\bar{\epsilon}^{\prime}=\epsilon-\bar{\epsilon}+i D \theta .
$$

This equation can be used to set $\epsilon-\bar{\epsilon}=0$ on $\mathcal{N}_{\star}$. Also, after solving this equation, the result $\theta$ on $\mathcal{N}_{\star}$ can be the initial value of Eq. (10). The value of $\mathrm{Q}$ on $\mathcal{N}_{\star}$ can be propagated from $\mathcal{S}_{\star}$ using the transport equation,

$$
D Q=-(\epsilon+\bar{\epsilon}) Q=-2 \epsilon Q
$$

that is,

$$
\partial_{v} Q=-2 \epsilon Q \text {. }
$$


Summarising, we have the following gauge restriction, which we employ exclusively in what follows:

Lemma 1 (Properties of the connection coefficients in Stewart's gauge) The NP frame in 1 can be chosen such that

$$
\begin{aligned}
& \kappa=v=\gamma=0, \\
& \rho=\bar{\rho}, \quad \mu=\bar{\mu}, \\
& \pi=\alpha+\bar{\beta}
\end{aligned}
$$

on $\mathcal{V}$ and, furthermore, with

$$
\epsilon-\bar{\epsilon}=0 \text { on } \mathcal{V} \cap \mathcal{N}_{\star}
$$

\subsubsection{Equations for the frame coefficients}

Taking into account the conditions on the spin connection coefficients given by (11a)(11c), it follows that the remaining commutators yield the equations

$$
\begin{aligned}
& \Delta C^{\mathcal{A}}=-(\bar{\tau}+\pi) P^{\mathcal{A}}-(\tau+\bar{\pi}) \bar{P}^{\mathcal{A}}, \\
& \Delta P^{\mathcal{A}}=-\mu P^{\mathcal{A}}-\bar{\lambda} \bar{P}^{\mathcal{A}}, \\
& D P^{\mathcal{A}}-\delta C^{\mathcal{A}}=(\rho+\epsilon-\bar{\epsilon}) P^{\mathcal{A}}+\sigma \bar{P}^{\mathcal{A}}, \\
& D Q=-(\epsilon+\bar{\epsilon}) Q, \\
& \bar{\delta} P^{\mathcal{A}}-\delta \bar{P}^{\mathcal{A}}=(\alpha-\bar{\beta}) P^{\mathcal{A}}-(\bar{\alpha}-\beta) \bar{P}^{\mathcal{A}}, \\
& \delta Q=(\tau-\bar{\pi}) Q .
\end{aligned}
$$

Remark 5 Equations (12a)-(12b) allow us to evolve the frame coefficients $C^{\mathcal{A}}$ and $P^{\mathcal{A}}$ off of the null hypersurface $\mathcal{N}_{\star}$. Equations (12c)-(12d) allow evolution of the coefficients $Q$ and $P^{\mathcal{A}}$ along the null generators of $\mathcal{N}_{\star}$. Finally (12e)-(12f) provide constraints for $Q$ and $P^{\mathcal{A}}$ on the spheres $\mathcal{S}_{u, v}$.

\section{The initial data for the CIVP}

In this section we analyse general aspects of the CIVP for the vacuum Einstein field equations on the null hypersurfaces $\mathcal{N}_{\star}$ and $\mathcal{N}_{\star}$ associated to the prescription of initial data for the evolution equations. The hierarchical structure of the Einstein field equations in Stewart's gauge allows the identification of a basic reduced initial data set $r_{\star}$ from which the full initial data on $\mathcal{N}_{\star} \cup \mathcal{N}_{\star}$ can be computed.

Lemma 2 (Freely specifiable data for the CIVP) Working in the gauge given by the frame choice 1 and Lemma 1, initial data for the vacuum Einstein field equations on $\mathcal{N}_{\star} \cup \mathcal{N}_{\star}$ can be computed (near $\mathcal{S}_{\star}$ ) from a reduced data set $\mathbf{r}_{\star}$ consisting of:

$$
\Psi_{0}, \epsilon+\bar{\epsilon} \text { on } \mathcal{N}_{\star},
$$




$$
\begin{aligned}
& \Psi_{4} \text { on } \mathcal{N}_{\star}, \\
& \lambda, \quad \sigma, \quad \mu, \quad \rho, \quad \pi, \quad P^{\mathcal{A}} \text { on } \mathcal{S}_{\star} .
\end{aligned}
$$

Proof The proof follows by inspection of the various intrinsic equations on $\mathcal{N}_{\star}, \mathcal{N}_{\star}$ and $\mathcal{S}_{\star}$. This inspection is done as follows:

Data on $\mathcal{S}_{\star}$. Since $P^{\mathcal{A}}$ are given, the operators $\delta$ and $\bar{\delta}$ are well defined on $\mathcal{S}_{\star}$ and intrinsic to this 2-dimensional hypersurface. From the definition of the connection coefficients $\alpha$ and $\beta$ it follows that the inner connection of $\mathcal{S}_{\star}$ is described by the combination $\alpha-\bar{\beta}$. This is readily computable from the data $P^{\mathcal{A}}$ on $\mathcal{S}_{\star}$. Thus, using $\alpha+$ $\bar{\beta}=\pi$, one can compute $\alpha$ and $\beta$. Noting that $Q=1$ on $\mathcal{S}_{\star} \subset \mathcal{N}_{\star}$, we obtain that $\pi=\bar{\tau}$ from (12f). Then we obtain all the values of connection coefficients on $\mathcal{S}_{\star}$. Thus, the constraint Eqs. (30q), (30j), (30n) of the structure equations can be used to compute the value of $\Psi_{1}, \Psi_{2}, \Psi_{3}$ on $\mathcal{S}_{\star}$. With that, all initial data for the connection coefficients and Weyl curvature on $\mathcal{S}_{\star}$ have been obtained.

Data on $\mathcal{N}_{\star}$ On the incoming null hypersurface $\mathcal{N}_{\star}$ we can obtain that $Q=1$ leads to $\tau=\bar{\pi}$ from Eq. (12f) and $\Delta=\partial_{u}$. Making use of the structure Eqs. (30g) and (30o), which can be reduced by the gauge condition, namely

$$
\begin{aligned}
& \frac{\partial \mu}{\partial u}=-\lambda \bar{\lambda}-\mu^{2}, \\
& \frac{\partial \lambda}{\partial u}=-\Psi_{4}-2 \lambda \mu,
\end{aligned}
$$

we can obtain the value of $\mu$ and $\lambda$ on $\mathcal{N}_{\star}$. Then the frame coefficients $P^{\mathcal{A}}$ on $\mathcal{N}_{\star}$ are computed using Eq. (12b) which takes the form

$$
\frac{\partial P^{\mathcal{A}}}{\partial u}=-\mu P^{\mathcal{A}}-\bar{\lambda} \bar{P}^{\mathcal{A}}
$$

Thus we can compute the $\delta$-direction derivative on $\mathcal{N}_{\star}$. Solving the structure Eqs. (30d), (30k) with the Bianchi identity Eq. (31d), namely

$$
\begin{aligned}
& -\frac{\partial \alpha}{\partial u}=\Psi_{3}+\beta \lambda+\alpha \bar{\mu}+\lambda \tau \\
& -\frac{\partial \beta}{\partial u}=\alpha \bar{\lambda}+\beta \mu+\mu \tau, \\
& \frac{\partial \Psi_{3}}{\partial u}-P^{\mathcal{A}} \frac{\partial \Psi_{4}}{\partial x^{\mathcal{A}}}=(4 \beta-\tau) \Psi_{4}-4 \mu \Psi_{3},
\end{aligned}
$$

together we can compute the value of $\alpha, \beta$ and $\Psi_{3}$ on $\mathcal{N}_{\star}$. Then Eq. (12a)

$$
\frac{\partial C^{\mathcal{A}}}{\partial u}=-(\bar{\tau}+\pi) P^{\mathcal{A}}-(\tau+\bar{\pi}) \bar{P}^{\mathcal{A}}
$$


reveals the value of the frame coefficients $C^{\mathcal{A}}$ on $\mathcal{N}_{\star}$. With the above information at hand one can use Eqs. (30a), (30i), (30r) and (31e):

$$
\begin{aligned}
& \frac{\partial \epsilon}{\partial u}=-\Psi_{2}-\beta \pi-\alpha \bar{\pi}-\alpha \tau-\pi \tau-\beta \bar{\tau}, \\
& P^{\mathcal{A}} \frac{\partial \tau}{\partial x^{\mathcal{A}}}-\frac{\partial \sigma}{\partial u}=\bar{\lambda} \rho+\mu \sigma-\bar{\alpha} \tau+\beta \tau+\tau^{2}, \\
& \bar{P}^{\mathcal{A}} \frac{\partial \tau}{\partial x^{\mathcal{A}}}-\frac{\partial \rho}{\partial u}=\Psi_{2}+\bar{\mu} \rho+\lambda \sigma+\alpha \tau-\bar{\beta} \tau+\tau \bar{\tau}, \\
& \frac{\partial \Psi_{2}}{\partial u}-P^{\mathcal{A}} \frac{\partial \Psi_{3}}{\partial x^{\mathcal{A}}}=\sigma \Psi_{4}+2(\beta-\tau) \Psi_{3}-3 \mu \Psi_{2}
\end{aligned}
$$

to compute the value of $\epsilon, \sigma, \rho$ and $\Psi_{2}$ on $\mathcal{N}_{\star}$. The Bianchi identity Eq. (31h)

$$
\frac{\partial \Psi_{1}}{\partial u}-P^{\mathcal{A}} \frac{\partial \Psi_{2}}{\partial x^{\mathcal{A}}}=-2 \mu \Psi_{1}-3 \tau \Psi_{2}+2 \sigma \Psi_{3},
$$

provides the value of $\Psi_{1}$ on $\mathcal{N}_{\star}$. With the results above, we can then compute the value of $\Psi_{0}$ from Eq. (31b)

$$
\frac{\partial \Psi_{0}}{\partial u}-P^{\mathcal{A}} \frac{\partial \Psi_{1}}{\partial x^{\mathcal{A}}}=-\mu \Psi_{0}-2(2 \tau+\beta) \Psi_{1}+3 \sigma \Psi_{2} .
$$

Data on $\mathcal{N}_{\star}$ From Eq. $(12 \mathrm{~d})$ one has that $\partial_{v} Q=-(\epsilon+\bar{\epsilon}) Q$ so that, using the value of $Q$ at $\mathcal{S}_{\star}$ one can compute the value of $Q$ on $\mathcal{N}_{\star}$. The structure Eqs. (30f) and (30m) give

$$
\begin{aligned}
& \frac{\partial \sigma}{\partial v}=\Psi_{0}+3 \epsilon \sigma-\bar{\epsilon} \sigma+2 \rho \sigma, \\
& \frac{\partial \rho}{\partial v}=2 \epsilon \rho+\rho^{2}+\sigma \bar{\sigma} .
\end{aligned}
$$

Solving these last equations one can obtain the value of $\sigma$ and $\rho$ on $\mathcal{N}_{\star}$. Then the value of $P^{\mathcal{A}}$ on $\mathcal{N}_{\star}$ can be computed using Eq. (12c) which in the present setting takes the form

$$
\frac{\partial P^{\mathcal{A}}}{\partial v}=\rho P^{\mathcal{A}}+\sigma \bar{P}^{\mathcal{A}}
$$

Then the structure Eqs. (30e), (301) and the Bianchi identity (31a), namely,

$$
\begin{aligned}
& P^{\mathcal{A}} \frac{\partial \epsilon}{\partial x^{\mathcal{A}}}-\frac{\partial \beta}{\partial v}=-\Psi_{1}+\bar{\alpha} \epsilon+\beta \bar{\epsilon}-\epsilon \bar{\pi}-\beta \rho-\alpha \sigma-\pi \sigma \\
& \bar{P}^{\mathcal{A}} \frac{\partial \epsilon}{\partial x^{\mathcal{A}}}-\frac{\partial \alpha}{\partial v}=2 \alpha \epsilon+\bar{\beta} \epsilon-\alpha \bar{\epsilon}-\epsilon \pi-\alpha \rho-\pi \rho-\beta \bar{\sigma} \\
& \bar{P}^{\mathcal{A}} \frac{\partial \Psi_{0}}{\partial x^{\mathcal{A}}}-\frac{\partial \Psi_{1}}{\partial v}=(4 \alpha-\pi) \Psi_{0}-2(2 \rho+\epsilon) \Psi_{1}
\end{aligned}
$$


provide us the value of $\alpha, \beta$ and $\Psi_{1}$ on $\mathcal{N}_{\star}$. Next, the structure Eq. (30b) which takes the form

$$
\frac{\partial \tau}{\partial v}=\Psi_{1}+\bar{\pi} \rho+\pi \sigma+\epsilon \tau-\bar{\epsilon} \tau+\rho \tau+\sigma \bar{\tau}
$$

gives us the value of $\tau$ on $\mathcal{N}_{\star}$. Similarly, the structure Eqs. (30h), (30p) and the Bianchi identity Eq. (31e)

$$
\begin{aligned}
& P^{\mathcal{A}} \frac{\partial \pi}{\partial x^{\mathcal{A}}}-\frac{\partial \mu}{\partial v}=-\Psi_{2}+\epsilon \mu+\bar{\epsilon} \mu+\bar{\alpha} \pi-\beta \pi-\pi \bar{\pi}-\mu \rho-\lambda \sigma, \\
& \bar{P}^{\mathcal{A}} \frac{\partial \pi}{\partial x^{\mathcal{A}}}-\frac{\partial \lambda}{\partial v}=3 \epsilon \lambda-\bar{\epsilon} \lambda-\alpha \pi+\bar{\beta} \pi-\pi^{2}-\lambda \rho-\mu \bar{\sigma}, \\
& \frac{\partial \Psi_{2}}{\partial v}-\bar{P}^{\mathcal{A}} \frac{\partial \Psi_{1}}{\partial x^{\mathcal{A}}}=-\lambda \Psi_{0}+2(\pi-\alpha) \Psi_{1}+3 \rho \Psi_{2}
\end{aligned}
$$

give us the value of $\mu, \lambda$ and $\Psi_{2}$ on $\mathcal{N}_{\star}$. Next, the Bianchi identity Eqs. (31g) and (31c)

$$
\begin{aligned}
& \frac{\partial \Psi_{3}}{\partial v}-\bar{P}^{\mathcal{A}} \frac{\partial \Psi_{2}}{\partial x^{\mathcal{A}}}=2(\rho-\epsilon) \Psi_{3}+3 \pi \Psi_{2}-2 \lambda \Psi_{1}, \\
& \bar{P}^{\mathcal{A}} \frac{\partial \Psi_{3}}{\partial x^{\mathcal{A}}}-\frac{\partial \Psi_{4}}{\partial v}=(4 \epsilon-\rho) \Psi_{4}-2(2 \pi+\alpha) \Psi_{3}+3 \lambda \Psi_{2},
\end{aligned}
$$

show us the value of $\Psi_{3}$ and $\Psi_{4}$ on $\mathcal{N}_{\star}$. Finally, we have obtained all the initial values on $\mathcal{N}_{\star} \cup \mathcal{N}_{\star}$ from the reduced data set $r_{\star}$.

\section{Rendall's local existence theory}

In order to apply the basic local existence theory for the CIVP as formulated by Rendall [13] (see also Section 12.5 of [28]), one has to extract a suitable symmetric hyperbolic evolution system from the Einstein field equations. The gauge introduced in Sect. 2.1 allows us to perform this reduction.

\subsection{Construction of the reduced evolution system}

In the following it will be convenient to group the components of the frame in the vector valued function

$$
\boldsymbol{e}^{t} \equiv\left(C^{\mathcal{A}}, P^{\mathcal{A}}, Q\right)
$$

the spin connection coefficients not fixed by the gauge in

$$
\Gamma^{t} \equiv(\epsilon, \pi, \beta, \mu, \alpha, \lambda, \tau, \sigma, \rho)
$$


and the independent components of the Weyl spinor as

$$
\Psi^{t} \equiv\left(\Psi_{0}, \Psi_{1}, \Psi_{2}, \Psi_{3}, \Psi_{4}\right)
$$

where superscript- $t$ denotes the operation of taking the transpose of a column vector.

A suitable symmetric hyperbolic system for the the frame components and the spin coefficients can be obtained from Eqs. (12a), (12b), (12d) and (30a), (30b), (30c), (30d), (30f), (30g), (30k), (30m), (30o), respectively. These can be written in the schematic form

$$
\begin{aligned}
& \mathcal{D}_{1} \boldsymbol{e}=\boldsymbol{B}_{1}(\boldsymbol{\Gamma}, \boldsymbol{e}) \boldsymbol{e}, \\
& \mathcal{D}_{2} \boldsymbol{\Gamma}=\boldsymbol{B}_{2}(\boldsymbol{\Gamma}, \boldsymbol{\Psi}) \boldsymbol{\Gamma},
\end{aligned}
$$

where $\mathcal{D}_{1}$ and $\mathcal{D}_{2}$ are matrix operators given by,

$$
\begin{aligned}
& \mathcal{D}_{1}=\operatorname{diag}(\Delta, \Delta, D), \\
& \mathcal{D}_{2}=\operatorname{diag}(\Delta, \Delta, \Delta, \Delta, \Delta, \Delta, D, D, D),
\end{aligned}
$$

and $\boldsymbol{B}_{1}, \boldsymbol{B}_{2}$ are smooth matrix-valued functions of their arguments whose explicit form will not be required in the subsequent analysis in this section.

The Bianchi identity Eqs. (31a)-(31h) can be reorganised as

$$
\mathcal{D}_{3} \boldsymbol{\Psi}=\boldsymbol{B}_{3} \boldsymbol{\Psi}
$$

where

$$
\mathcal{D}_{3}=\left(\begin{array}{ccccc}
\Delta & -\delta & 0 & 0 & 0 \\
-\bar{\delta} & D+\Delta & -\delta & 0 & 0 \\
0 & -\bar{\delta} & D+\Delta & -\delta & 0 \\
0 & 0 & -\bar{\delta} & D+\Delta & -\delta \\
0 & 0 & 0 & -\bar{\delta} & D
\end{array}\right)
$$

and $\boldsymbol{B}_{\mathbf{3}}=\boldsymbol{B}_{\mathbf{3}}(\boldsymbol{\Gamma})$. Writing

$$
\mathcal{D}_{3}=A_{3}^{\mu} \partial_{\mu}
$$

one has that

$$
\begin{aligned}
& \boldsymbol{A}_{3}^{v}=\operatorname{diag}(0,1,1,1,1), \\
& \boldsymbol{A}_{3}^{u}=\operatorname{diag}(Q, Q, Q, Q, 0),
\end{aligned}
$$


and

$$
\boldsymbol{A}_{3}^{\mathcal{A}}=\left(\begin{array}{ccccc}
0 & -P^{\mathcal{A}} & 0 & 0 & 0 \\
-\bar{P}^{\mathcal{A}} & C^{\mathcal{A}} & -P^{\mathcal{A}} & 0 & 0 \\
0 & -\bar{P}^{\mathcal{A}} & C^{\mathcal{A}} & -P^{\mathcal{A}} & 0 \\
0 & 0 & -\bar{P}^{\mathcal{A}} & C^{\mathcal{A}} & -P^{\mathcal{A}} \\
0 & 0 & 0 & -\bar{P}^{\mathcal{A}} & C^{\mathcal{A}}
\end{array}\right)
$$

The evolution system (13) for the components of the Weyl tensor are obtained through the combinations (31b), (31h)-(31a), (31e)+(31f), (31d)+(31g) and -(31c) respectively. It can be readily verified that the matrices $\boldsymbol{A}_{3}^{\mu}$ are Hermitian. Moreover,

$$
\boldsymbol{A}_{3}^{\mu}\left(l_{\mu}+n_{\mu}\right)=\operatorname{diag}(1,2,2,2,1)
$$

is clearly positive definite. We can summarise the above discussion with:

Lemma 3 (The evolution system) The evolution system

$$
\begin{aligned}
& \mathcal{D}_{1} \boldsymbol{e}=\boldsymbol{B}_{1} \boldsymbol{e}, \\
& \mathcal{D}_{2} \boldsymbol{\Gamma}=\boldsymbol{B}_{2} \boldsymbol{\Gamma}, \\
& \mathcal{D}_{3} \boldsymbol{\Psi}=\boldsymbol{B}_{3} \boldsymbol{\Psi},
\end{aligned}
$$

implied by the NP field equations written in Stewart's gauge (see Sect. 2.1) is symmetric hyperbolic with respect to the direction given by $\tau^{a}=l^{a}+n^{a}$.

Remark 6 In the following, making use of the standard terminology, we call the evolution system the reduced Einstein field equations.

Remark 7 The symmetric hyperbolicity of the reduced Eqs. (14a)-(14c) is the key structural property which allows us to employ Rendall's local existence strategy-see the discussion in Sect. 4.2 below.

As the hyperbolic reduction leading to the previous result makes use of a subset of the NP equations, it is also key to have a propagation of the constraints result for the discarded equations. Making use of analysis similar to the one discussed in Section 12.5 of [28] one obtains the following:

Proposition 1 (Propagation of the constraints) A solution of the reduced vacuum Einstein field Eqs. (14a)-(14c) on a neighbourhood $\mathcal{V}$ of $\mathcal{S}_{\star}$ on $J^{+}\left(\mathcal{S}_{\star}\right)$, the causal future of $\mathcal{S}_{\star}$, that coincides with initial data on $\mathcal{N}_{\star} \cup \mathcal{N}_{\star}$ satisfying the vacuum Einstein equations is a solution to the vacuum Einstein field equations on $\mathcal{V}$.

Remark 8 A consequence of the propagation of the constraints, once local existence has been established, is that we may use any combination of the NP field equations in their gauge simplified form in the required subsequent analysis. For example, from this point on we have $\pi=\alpha+\bar{\beta}$, and hence discard $\pi$ or view it as a shorthand in what follows. 


\subsection{Computation of the formal derivatives on $\mathcal{N}_{\star} \cup \mathcal{N}_{\star}$}

As already mentioned, Rendall's approach to the local existence of solutions to the characteristic problem for symmetric hyperbolic systems makes use of an auxiliary Cauchy problem on an auxiliary spacelike hypersurface

$$
S \equiv\left\{p \in \mathbb{R} \times \mathbb{R} \times \mathbb{S}^{2} \mid v(p)+u(p)=0\right\}
$$

which contains the 2-dimensional surface $\mathcal{S}_{\star}-$ see Sect. 2.1.1. The formulation of this problem crucially depends on Whitney's extension theorem. To apply this extension theorem it is necessary to be able to evaluate all derivatives (interior and transverse) of the initial data on $\mathcal{N}_{\star} \cup \mathcal{N}_{\star}$. A discussion of the ideas behind Rendall's approach can be found in Section 12.5 of [28]. For completeness, a formulation of Rendall's result is given below:

Theorem 1 (Local existence for the CIVP, Rendall) Let $\mathcal{N}_{\star}$ and $\mathcal{N}_{\star}$ denote two characteristic hypersurfaces for the symmetric hyperbolic system

$$
\mathbf{A}^{\mu}(x, \mathbf{u}) \partial_{\mu} \mathbf{u}=\mathbf{B}(x, \mathbf{u})
$$

with smooth, freely specifiable data on $\mathcal{N}_{\star}$ and $\mathcal{N}_{\star}$ such that all (formal) derivatives of $\mathbf{u}$ on $\mathcal{N}_{\star} \cup \mathcal{N}_{\star}$ to any desired order can be computed in a neighbourhood $\mathcal{W} \subset \mathcal{N}_{\star} \cup \mathcal{N}_{\star}$ of $\mathcal{N}_{\star} \cap \mathcal{N}_{\star}$. Then there exists a unique solution $\mathbf{u}$ to the CIVP in a neighbourhood $\mathcal{V}$ of $\mathcal{N}_{\star} \cap \mathcal{N}_{\star}$ with $u \geq 0, v \geq 0$.

An important property of the NP equations in Stewart's gauge is that they allow the computation of the (formal) derivatives of all the fields to any order from the reduced data $\mathbf{r}_{\star}$ provided in Lemma 2 . This property is discussed in the next paragraphs.

Computation of formal derivatives on $\mathcal{N}_{\star}$ To compute the formal derivatives on $\mathcal{N}_{\star}$ one first observes that the partial derivatives $\partial_{v}, \partial_{2}, \partial_{3}$ are interior whereas $\partial_{u}$ is transverse. In this case, direct inspection shows that except for

$$
\partial_{u} Q, \quad \partial_{u} \tau, \quad \partial_{u} \Psi_{4},
$$

all $\partial_{u}$-derivatives of the unknowns in the vectors $\boldsymbol{e}, \boldsymbol{\Gamma}, \boldsymbol{\Psi}$ can be computed using the structure Eqs. (12a), (12b), the NP Ricci identities (30a), (30c), (30d), (30g), (30i), (30k), (30o), (30r), and the Bianchi identities (31b), (31d), (31f) and (31h).

To obtain these exceptional cases one first applies $Q \boldsymbol{\partial}_{u}$ to both sides of Eqs. (12d), (30b) and (31c) to obtain

$$
\begin{aligned}
& Q \partial_{v}\left(\partial_{u} Q\right)=-Q^{2} \partial_{u}(\epsilon+\bar{\epsilon})-Q(\epsilon+\bar{\epsilon}) \partial_{u} Q, \\
& Q \partial_{v}\left(\partial_{u} \tau\right)=L\left(\partial_{u} \tau\right) \\
& Q \partial_{v}\left(\partial_{u} \Psi_{4}\right)-Q \partial_{u} \bar{P}^{\mathcal{A}} \partial_{\mathcal{A}} \Psi_{3}-Q \bar{P}^{\mathcal{A}} \partial_{u} \partial_{\mathcal{A}} \Psi_{3}=M\left(\partial_{u} \Psi_{4}\right),
\end{aligned}
$$

where $L, M$ are smooth functions of $\{\boldsymbol{e}, \boldsymbol{\Gamma}, \Psi\}$ and their $\boldsymbol{n}$-direction derivatives. One can regard the above equations as first order linear ordinary differential equations 
for $\partial_{u} Q, \partial_{u} \tau, \partial_{u} \Psi_{4}$ along the generators of $\mathcal{N}_{\star}$. Since we have all the initial values of the components of $\{\boldsymbol{e}, \boldsymbol{\Gamma}, \boldsymbol{\Psi}\}$ on $\mathcal{N}_{\star} \cup \mathcal{N}_{\star}$, we can compute the initial value of $\partial_{u} Q, \partial_{u} \tau, \partial_{u} \Psi_{4}$ on $\mathcal{S}_{\star}$. The general results for the existence theorem of ordinary differential equations ensures that the above equation system can be solved in a neighbourhood of $\mathcal{S}_{\star}$. In the following, we assume that the initial data provided is such that it yields a uniform existence domain for the solutions to the transport equations - this is a major assumption on the initial data in this construction. Accordingly, all the first transverse derivatives on $\mathcal{N}_{\star}$ can be explicitly computed. The higher order $\partial_{u}$-derivatives can be computed in a similar way. Throughout it is assumed that the neighbourhood on which this construction can be done in uniform for any order of the derivatives.

Computation of formal derivatives on $\mathcal{N}_{\star}$ The analysis of the formal derivatives on $\mathcal{N}_{\star}$ is almost the mirror image of that on $\mathcal{N}_{\star}^{\star}$. In this case $\partial_{u}, \partial_{2}, \partial_{3}$ are interior while $\partial_{v}$ is transverse. Accordingly, except for

$$
\partial_{v} C^{A}, \quad \partial_{v} \epsilon, \quad \partial_{v} \Psi_{0},
$$

all $\partial_{v}$-derivatives of the components of $\{\boldsymbol{e}, \boldsymbol{\Gamma}, \boldsymbol{\Psi}\}$ can be computed using the structure equations (12c)-(12d), the Ricci identities and the Bianchi identity. Applying the directional derivative $D=\partial_{v}+C^{A} \partial_{A}$ to both sides of Eqs. (12a), (30a) and (31b) one obtains equations which can be regarded as first order linear ordinary differential equations for $\partial_{v} C^{A}, \partial_{v} \epsilon, \partial_{v} \Psi_{0}$. The solutions to these equations can be obtained from the initial values prescribed on $\mathcal{S}_{\star}$. Thus, all transverse derivatives can be computed in a neighbourhood of $\mathcal{S}_{\star}$ on $\mathcal{N}_{\star}$. A similar procedure applies to higher order derivatives.

The analysis described in the previous paragraph proves the following lemma:

Lemma 4 (Computation of formal derivatives) Any arbitrary formal derivatives of the unknown functions $\{\boldsymbol{e}, \boldsymbol{\Gamma}, \boldsymbol{\Psi}\}$ on $\mathcal{N}_{\star} \cup \mathcal{N}_{\star}$ can be computed from the prescribed initial data $\boldsymbol{r}_{\star}$ for the reduced vacuum Einstein field equations on $\mathcal{N}_{\star} \cap \mathcal{N}_{\star}$.

Combining the analysis above and applying Rendall's reduction strategy for the CIVP for symmetric hyperbolic systems (see e.g. Section 12.5 of [28]) one obtains the following local existence result in a neighbourhood of $\mathcal{S}_{\star}=\mathcal{N}_{\star} \cup \mathcal{N}_{\star}$ :

Theorem 2 (Existence and uniqueness to the characteristic problem) Given a smooth reduced initial data set $\boldsymbol{r}_{\star}$ for the vacuum Einstein field equations on $\mathcal{N}_{\star} \cup \mathcal{N}_{\star}$, there exists a unique smooth solution of the vacuum Einstein field equations in a neighbourhood $\mathcal{V}$ of $\mathcal{S}_{\star}$ on $J^{+}\left(\mathcal{S}_{\star}\right)$ which implies the prescribed initial data on $\mathcal{N}_{\star} \cup \mathcal{N}_{\star}$.

Remark 9 The proof of the above result has two distinct parts. In a first stage one uses Rendall's reduction procedure to show the existence of a solution in a neighbourhood of $\mathcal{V}$. In a second stage one shows that this solution to the reduced equations implies, in fact, a solution to the full Einstein field equations. This part of the argument relies on the propagation of the constraints as given in Proposition 1. 


\section{Setting-up Luk's strategy}

In this section we begin the implementation of Luk's strategy to obtain an improved existence interval for the solutions to the CIVP for the NP field equations in Stewart's gauge.

\subsection{Outline and main strategy}

As the argument leading to the improved existence result for the CIVP is lengthy, we provide here a summary of the role of the various lemmas and propositions and a discussion of how they fit into the overall analysis. The whole scheme is based on the use of sequentially more sophisticated a priori estimates of an arbitrary solution that, ultimately, arrives at a contradiction giving us the desired result.

Step 0 Estimates for the components of the frame. The basic step in the construction is to obtain estimates on the components of the frame. This can be done by assuming control on the $L^{\infty}$-norm on the spheres $\mathcal{S}_{u, v}$ of a number of spin connection coefficients by a constant $\Delta_{\Gamma}$. A peculiarity of the analysis is that one needs to introduce a certain derivative (to be denoted by $\chi$ ) of the components of the frame as an unknown to quick-start the argument - this quantity, which is at the level of the spin connection coefficients, does not arise in the original NP formalism. The key result in this step is Lemma 5 in which the frame coefficients $Q$ and $P^{\mathcal{A}}$ are controlled by their initial data and Lemma 6 in which the frame coefficients $C^{\mathcal{A}}$ are controlled along the short direction.

The bounds on the components of the frame allow us to control in a systematic and streamlined manner the solutions to transport equations along null directions in terms of integral quantities over the spheres $\mathcal{S}_{u, v}$. The technical results required to this end are presented in Lemmas 7 and 8. From these, more specific results valid for $L^{p}$ and $L^{\infty}$ norms are given in Propositions 2, 3, 5 and 6. Within our geometric setup and gauge these results are fairly general and are used repeatedly in the subsequent steps of the procedure.

Step 1 Estimates for the connection coefficients. With the general technology to study transport equations along the generators of light cones has been established, one can proceed to control the spin connection coefficients. The key idea of this analysis is the integration of the transport equations implied by the Ricci identities. In a first step, in Proposition 7, assuming control on the supremum norm of the third angular derivatives of the NP connection coefficient $\tau$ and on the components of the curvature one obtains control on the supremum norm of the various connection coefficients and $\tau$ itself. This result is used in turn in Proposition 8 to obtain control on the $L^{4}$-norms of the connection coefficients and the $L^{2}$-norm of their derivatives in Proposition 9. Step 2 First estimate for the curvature. A first estimate for the components of the Weyl tensor is given in Proposition 10. In this result one assumes control of the components of the Weyl tensor along the light cones and of the $L^{2}$-norm of the third angular derivatives of the connection coefficient $\tau$ on the spheres to obtain control of the components of the Weyl tensor on the spheres. 
The results of the steps 1 and 2 are conveniently summarised in Proposition 11 in which an assumed control on the components of the curvature along light cones and of the $L^{2}$-norm of the third angular derivatives of $\tau$ is used to obtain control on the spheres $\mathcal{S}_{u, v}$ of various norms of the connection and its derivatives and of the components of the curvature.

Step 3 Improved estimate for the connection. In the next step one obtains an improved estimate for the connection in which the third angular derivatives of the connection, including $\tau$, are controlled assuming control only on the curvature along the light cones. This result is given in Proposition 12.

Step 4 Main estimates for the curvature. At this point we are in a position to run the central part of the argument, which depends crucially on the particular structure of the Bianchi identities. General inequalities for integrals of the various components of the Weyl tensor implied by the Bianchi identities are given in Propositions 13, 14 and 15 and 16. The whole argument is wrapped up in Proposition 17 in which, under the boundedness of the connection and the curvature on the initial null hypersurfaces one obtains control of the curvature on later null hypersurfaces. This is the crucial estimate which allows us to close the lengthy boostrap argument.

Final step. Last slice argument The control of various norms of the connection and curvature obtained in the previous steps do not provide, by themselves, the improved existence result. For this, we make use of a last slice argument in which one argues by contradiction under the assumption that the solution to the evolution equations breaks down at some point. The estimates of the previous steps show that this assumption leads to a contradiction.

\subsection{Definitions and conventions}

In this section we set up the conventions for the various norms that will be used in the subsequent analysis.

Integration In the following let $\phi$ denote a scalar field. For conciseness, we will often use the notation

$$
\int_{\mathcal{S}_{u, v}} \phi \equiv \int_{\mathcal{S}_{u, v}} \phi \mathrm{d} \sigma
$$

to denote integration on the spheres $\mathcal{S}_{u, v}$ of constant $u$ and $v$. In the previous expression $\mathrm{d} \sigma \equiv \sqrt{|\operatorname{det} \sigma|} \mathrm{d} x^{2} \mathrm{~d} x^{3}$ denotes the volume element of the induced metric $\sigma$ on $\mathcal{S}_{u, v}$. On the truncated causal diamonds $\mathcal{D}_{u, v}^{t}$ we define integration using the volume form of the spacetime metric,

$$
\begin{aligned}
\int_{\mathcal{D}_{u, v}^{t}} \phi & \equiv \int_{0}^{u} \int_{0}^{\tilde{v}} \int_{\mathcal{S}_{u^{\prime}, v^{\prime}}} \phi \sqrt{|\operatorname{det} \boldsymbol{g}|} \mathrm{d} x^{2} \mathrm{~d} x^{3} \mathrm{~d} v^{\prime} \mathrm{d} u^{\prime} \\
& =\int_{0}^{u} \int_{0}^{\tilde{v}} \int_{\mathcal{S}_{u^{\prime}, v^{\prime}}} Q^{-1} \phi \sqrt{|\operatorname{det} \sigma|} \mathrm{d} x^{2} \mathrm{~d} x^{3} \mathrm{~d} v^{\prime} \mathrm{d} u^{\prime},
\end{aligned}
$$


with $\tilde{v} \equiv \min (v, t-u)$. We will denote integration over the complete causal diamond in the obvious manner by the natural omission of the superscript $t$ on $\mathcal{D}_{u, v}^{t}$. As there are no canonical volume forms on the null hypersurfaces $\mathcal{N}_{u}$ and $\mathcal{N}_{v}$ we define, for convenience the following:

$$
\begin{aligned}
\int_{\mathcal{N}_{u}(0, v)} \phi & \equiv \int_{0}^{v} \int_{S_{u, v^{\prime}}} \phi \sqrt{|\operatorname{det} \sigma|} \mathrm{d} x^{2} \mathrm{~d} x^{3} \mathrm{~d} v^{\prime}, \\
\int_{\mathcal{N}_{v}(0, u)} \phi & \equiv \int_{0}^{u} \int_{S_{u^{\prime}, v}} \phi \sqrt{|\operatorname{det} \sigma|} \mathrm{d} x^{2} \mathrm{~d} x^{3} \mathrm{~d} u^{\prime} .
\end{aligned}
$$

We will often use the notation

$$
\int_{\mathcal{N}_{u}^{t}} \phi \equiv \int_{\mathcal{N}_{u}\left(I^{t}\right)} \phi, \quad \int_{\mathcal{N}_{v}^{t}} \phi \equiv \int_{\mathcal{N}_{v}[0, \varepsilon]^{t}} \phi
$$

where $I^{t} \equiv\left[0, \min \left(v_{\bullet}, t-u\right)\right]$, with $v_{\bullet} \in \mathbb{R}^{+}$, denotes the truncated long integration interval. Similarly, the interval $[0, \varepsilon]^{t} \equiv[0, \min (\varepsilon, t-v)]$ will be called the truncated short integration interval. Dropping the superscript $t$ we define the full long and short integration intervals, $I$ and $[0, \varepsilon]$ respectively, and the norms on the full outgoing and incoming slices in the natural way.

Norms Keeping the above conventions for integration in mind, we can now define the various norms to be used in our analysis. As before, let $\phi$ define a scalar field. For $1 \leq p<\infty$ we define the $L^{p}$-norms

$$
\begin{aligned}
\|\phi\|_{L^{p}\left(\mathcal{S}_{u, v}\right)} & \equiv\left(\int_{\mathcal{S}_{u, v}}|\phi|^{p}\right)^{1 / p}, \quad\|\phi\|_{L^{p}\left(\mathcal{N}_{u}^{t}\right)} \\
& \equiv\left(\int_{\mathcal{N}_{u}^{t}}|\phi|^{p}\right)^{1 / p}, \quad\|\phi\|_{L^{p}\left(\mathcal{N}_{v}^{t}\right)} \equiv\left(\int_{\mathcal{N}_{v}^{t}}|\phi|^{p}\right)^{1 / p} .
\end{aligned}
$$

The $L^{\infty}$-norm is defined by

$$
\|\phi\|_{L^{\infty}\left(\mathcal{S}_{u, v}\right)} \equiv \sup _{\mathcal{S}_{u, v}}|\phi|
$$

For a tensor field $\phi_{a_{1} \ldots a_{p}}$ on the 2-sphere, we define

$$
\begin{aligned}
\|\phi\|_{L^{p}\left(\mathcal{S}_{u, v}\right)} & \equiv\left(\int_{\mathcal{S}_{u, v}}\langle\phi, \phi\rangle_{\sigma}^{p / 2}\right)^{1 / p},\|\phi\|_{L^{p}\left(\mathcal{N}_{u}^{t}\right)} \\
& \equiv\left(\int_{\mathcal{N}_{u}^{t}}\langle\phi, \phi\rangle_{\sigma}^{p / 2}\right)^{1 / p},\|\phi\|_{L^{p}\left(\mathcal{N}_{v}^{t}\right)} \equiv\left(\int_{\mathcal{N}_{v}^{t}}\langle\phi, \phi\rangle_{\sigma}^{p / 2}\right)^{1 / p},
\end{aligned}
$$


where $\langle\phi, \phi\rangle_{\sigma} \equiv \sigma^{a_{1} b_{1}} \ldots \sigma^{a_{p} b_{p}} \bar{\phi}_{a_{1}, \ldots, a_{p}} \phi_{b_{1}, \ldots, b_{p}}$. As in the definition of the integrals, suppresion of the label $t$ denotes taking the norms over the full long and short integration intervals.

Integration by parts In the following we denote by $\not \nabla$ the covariant derivative of the induced metric $\sigma$ on the spheres $\mathcal{S}_{u, v}$ of constant $u$ and $v$. Similarly, $\Delta$ will denote the associated Laplacian. As these spheres have no boundary we have

$$
\begin{aligned}
\|\not \nabla \phi\|_{L^{2}\left(\mathcal{S}_{u, v}\right)}^{2} & =\int_{\mathcal{S}_{u, v}} \sigma^{a b} \nabla_{a} \phi \nabla_{b} \bar{\phi}=\int_{\mathcal{S}_{u, v}} \nabla_{a}\left(\sigma^{a b} \phi \nabla_{b} \bar{\phi}\right)-\int_{\mathcal{S}_{u, v}} \phi \bar{\phi} \bar{\phi} \\
& =-\int_{\mathcal{S}_{u, v}} \phi \phi \bar{\phi} \leq 2\left(\int_{\mathcal{S}_{u, v}}|\phi|^{2}\right)^{1 / 2}\left(\int_{\mathcal{S}_{u, v}}\left|\nabla^{2} \phi\right|^{2}\right)^{1 / 2}
\end{aligned}
$$

where in the last step inequality (35) in "Appendix C" has been used. Integrating over $\langle\phi, \pi\rangle_{\sigma}$ over two-spheres naturally defines an inner product, so we similarly obtain,

$$
\begin{aligned}
\|\not \nabla \phi\|_{L^{2}\left(\mathcal{S}_{u, v}\right)} & \leq\|\phi\|_{L^{2}\left(\mathcal{S}_{u, v}\right)}+\left\|\nabla^{2} \phi\right\|_{L^{2}\left(\mathcal{S}_{u, v}\right)}, \\
\left\|\not \nabla^{2} \phi\right\|_{L^{2}\left(\mathcal{S}_{u, v}\right)} & \leq\|\not \nabla \phi\|_{L^{2}\left(\mathcal{S}_{u, v}\right)}+\left\|\nabla^{3} \phi\right\|_{L^{2}\left(\mathcal{S}_{u, v}\right)} .
\end{aligned}
$$

\subsection{Estimates for the components of the frame}

As a preliminary step we now show that, assuming the components of the connection coefficients are controlled by a basic boostrap assumption, it is possible to estimate the components of the NP frame in terms of the size of its initial data on $\mathcal{N}_{\star} \cup \mathcal{N}_{\star}$. The key observation in the argument is that the structure equations provide $\Delta$-equations for all the components of the frame. Given our particular choice of gauge, these equations are essentially ordinary differential equations with respect to the coordinate $u$. In fact as the structure equations form a neat hierarchy, they can be integrated sequentially. The quantity,

$$
\Delta_{e_{\star}} \equiv \sup _{\mathcal{N}_{\star}, \mathcal{N}_{\star}}\left(|Q|,\left|Q^{-1}\right|,\left|C^{\mathcal{A}}\right|,\left|P^{\mathcal{A}}\right|\right)
$$

will be used to measure of the size of the initial data of the coefficients of the frame. Throughout, given that the procedure has only a finite number of steps we denote all constants depending on the initial data generically by $C\left(\Delta_{e_{\star}}\right)$-the latter corresponds to the largest constant arising in the various steps. For convenience in the subsequent discussion let

$$
\chi \equiv \Delta \log Q
$$

The scalar $\chi$, being a derivative of a component of the frame is at the same level of the connection coefficients. It provides a component of the connection which does not arise in the original NP formalism, but is needed to obtain a complete set of $\Delta$ 
equations for the frame. A direct computation using the definition of $\chi=\Delta \log Q$ and the NP Ricci identities yields

$$
D \chi=\Psi_{2}+\bar{\Psi}_{2}+2 \alpha \tau+2 \bar{\beta} \tau+2 \bar{\alpha} \bar{\tau}+2 \beta \bar{\tau}+2 \tau \bar{\tau}-(\epsilon+\bar{\epsilon}) \chi .
$$

The initial data of $\chi$ on $\mathcal{N}_{\star}$ is 0 due to the gauge choice that $Q=1$ on $\mathcal{N}_{\star}$. On $\mathcal{N}_{\star}$, making use of the information of $\alpha, \beta, \tau, \epsilon$ and $\Psi_{2}$ obtained in Lemma 2, one can compute the value of $\chi$ with Eq. (16). It will also be convenient to define,

$$
\varpi \equiv \beta-\bar{\alpha}
$$

corresponding to the only independent component of the connection on the spheres $\mathcal{S}_{u, v}$. As mentioned above, the proof is based on demonstrating a priori estimates for an arbitrary solution and consequently demonstrating that any such solution must extend to a neighborhood of $\mathcal{N}_{\star} \cup \mathcal{N}_{\star}$. We therefore now introduce the following, which will be initially guaranteed on a sufficiently small diamond by Theorem 1 , and will be employed in most of what follows:

Assumption 1 (Assumption to control the coefficients of the frame) Assume that we have a solution to the vacuum EFEs in Stewart's gauge satisfying,

$$
\|\{\mu, \lambda, \alpha, \beta, \tau, \chi\}\|_{L^{\infty}\left(\mathcal{S}_{u, v}\right)} \leq \Delta_{\Gamma},
$$

on a truncated causal diamond $\mathcal{D}_{u, v_{\bullet}}^{t}$, where $\Delta_{\Gamma}$ is some constant.

Step 1 Work under Assumption 1. Integrating the definition of $\chi=\Delta \log Q$ in the short (i.e. $u$ ) direction along an incoming null geodesic one readily finds that,

$$
\left|Q-Q_{\star}\right|=\left|\int_{0}^{\varepsilon} \chi \mathrm{d} u\right| \leq \int_{0}^{\varepsilon}|\chi| \mathrm{d} u \leq \int_{0}^{\varepsilon} \Delta_{\Gamma} \mathrm{d} u=\Delta_{\Gamma} \varepsilon
$$

for any $v$. It follows that

$$
\left\|Q-Q_{\star}\right\|_{L^{\infty}\left(\mathcal{S}_{u, v}\right)} \leq \Delta_{\Gamma} \varepsilon
$$

Hence, one can find a constant $C$ depending on the initial data such that

$$
Q^{-1}, Q \leq C\left(\Delta_{e_{\star}}\right)
$$

Step 2 We now integrate the components $P^{\mathcal{A}}$ in the short direction using Eq. (12b). It follows then that

$$
\begin{aligned}
\partial_{u}\left|P^{\mathcal{A}}\right|^{2} & =\partial_{u}\left(P^{\mathcal{A}} \bar{P}^{\mathcal{A}}\right)=P^{\mathcal{A}} \partial_{u} \bar{P}^{\mathcal{A}}+\bar{P}^{\mathcal{A}} \partial_{u} P^{\mathcal{A}} \\
& =-Q^{-1}\left(P^{\mathcal{A}}\left(\bar{\mu} \bar{P}^{\mathcal{A}}+\lambda P^{\mathcal{A}}\right)+\bar{P}^{\mathcal{A}}\left(\mu P^{\mathcal{A}}+\bar{\lambda} \bar{P}^{\mathcal{A}}\right)\right) \\
& =-Q^{-1}\left(\bar{\mu}\left|P^{\mathcal{A}}\right|^{2}+\lambda\left(P^{\mathcal{A}}\right)^{2}+\mu\left|P^{\mathcal{A}}\right|^{2}+\bar{\lambda}\left(\bar{P}^{\mathcal{A}}\right)^{2}\right)
\end{aligned}
$$




$$
\leq Q^{-1}(\mu+\bar{\mu}+\lambda+\bar{\lambda})\left|P^{\mathcal{A}}\right|^{2}
$$

In the previous chain of inequalities it is understood that there is no summation on the repeated indices $\mathcal{A}$. From the last inequality one readily concludes that

$$
\partial_{u} \ln \left|P^{\mathcal{A}}\right|^{2} \leq 4 Q^{-1} \Delta_{\Gamma}
$$

so that

$$
\left|P^{\mathcal{A}}\right|^{2} \leq\left|P_{\star}^{\mathcal{A}}\right|^{2} \exp \left(4 C\left(\Delta_{e_{\star}}\right) \Delta_{\Gamma} \varepsilon\right)
$$

As $\varepsilon$ is arbitrary, we can choose it so that

$$
\left|P^{\mathcal{A}}\right| \leq C\left(\Delta_{e_{\star}}\right) \text {, for any } u \text { and fixed } v \text {. }
$$

The analysis of Steps 1 and 2 can be summarised in the following:

Lemma 5 [Control on the components of the frame. I] Under Assumption 1, if $\varepsilon>0$ is sufficiently small, there exists a constant $C$ depending on the size of the initial data such that

$$
Q^{-1}, Q \leq C\left(\Delta_{e_{\star}}\right), \quad\left\|P^{\mathcal{A}}\right\|_{L^{\infty}\left(\mathcal{S}_{u, v}\right)} \leq C\left(\Delta_{e_{\star}}\right),
$$

on $\mathcal{D}_{u, v_{\bullet}}^{t}$.

A direct consequence of this result is that one can control the components of the induced metric on the spheres $S_{u, v}$ and associated concomitants. This follows from the relation

$$
\sigma^{\mathcal{A B}}=-P^{\mathcal{A}} \bar{P}^{\mathcal{B}}-P^{\mathcal{B}} \bar{P}^{\mathcal{A}}
$$

Corollary 1 (control on the metric of $\mathcal{S}_{u, v}$ ) If $\varepsilon>0$ is sufficiently small there exist non-negative constants $c\left(\Delta_{e_{\star}}\right)$ and $C\left(\Delta_{e_{\star}}\right)$ such that,

$$
\left|\sigma^{\mathcal{A B}}\right|,\left|\sigma_{\mathcal{A B}}\right| \leq C\left(\Delta_{e_{\star}}\right), \quad c\left(\Delta_{e_{\star}}\right) \leq|\operatorname{det} \sigma| \leq C\left(\Delta_{e_{\star}}\right) .
$$

Moreover, one also has that

$$
\sup _{u, v}\left|\operatorname{Area}\left(\mathcal{S}_{u, v}\right)-\operatorname{Area}\left(\mathcal{S}_{0, v}\right)\right| \leq C\left(\Delta_{e_{\star}}\right) \Delta_{\Gamma} \varepsilon
$$

on $\mathcal{D}_{u, v}^{t}$. Consequently the area of $\mathcal{S}_{u, v}$ is bounded above by a constant depending in initial data in the same region, for $\varepsilon$ sufficiently small. 
Step 3 One can now use Eq. (12a) to integrate the coefficients $C^{\mathcal{A}}$. By a procedure similar to that used in the previous steps one has,

$$
\begin{aligned}
\left|C^{\mathcal{A}}-C_{\star}^{\mathcal{A}}\right| & =\left|\int_{0}^{\epsilon} Q^{-1}\left((\bar{\tau}+\pi) P^{\mathcal{A}}+(\tau+\bar{\pi}) \bar{P}^{\mathcal{A}}\right) \mathrm{d} u\right| \\
& \leq C\left(\Delta_{e_{\star}}\right) \int_{0}^{\epsilon}\left|(\bar{\tau}+\pi) P^{\mathcal{A}}+(\tau+\bar{\pi}) \bar{P}^{\mathcal{A}}\right| \mathrm{d} u \\
& \leq 2 C\left(\Delta_{e_{\star}}\right) \int_{0}^{\epsilon}|\bar{\tau}+\pi|\left|P^{\mathcal{A}}\right| \mathrm{d} u \leq 2 C\left(\Delta_{e_{\star}}\right)^{2} \Delta_{\Gamma} \varepsilon .
\end{aligned}
$$

Here $\pi$ should be viewed as a shorthand for $\pi=\alpha+\bar{\beta}$. Since $C_{\star}^{\mathcal{A}}=0$ on $\mathcal{N}_{\star}$, we arrive at:

Lemma 6 (Control on the components of the frame. II) Under Assumption 1, if $\varepsilon>0$ is sufficiently small, then there is a constant $C\left(\Delta_{e_{\star}}\right)$ depending only on the initial data such that choosing $\varepsilon$ suitably, one has $\left\|C^{\mathcal{A}}\right\|_{L^{\infty}\left(\mathcal{S}_{u, v}\right)} \leq C\left(\Delta_{e_{\star}}\right)$ on $\mathcal{D}_{u, v_{\bullet}}^{t}$.

\subsection{General estimates for transport equations}

The purpose of this section is to develop a general set of tools that allow us to obtain estimates from the transport equations on hypersurfaces of constant $u$ or $v$. The prototype of these transport equations are the NP Ricci identities (30a)-(30r). The results of this section do not depend on Assumption 1 unless explicitly stated.

Derivatives of integrals over $\mathcal{S}_{u, v}$. We are mostly interested on integral estimates over the spheres $\mathcal{S}_{u, v}$ and how they evolve along null directions. In the following we will systematically need to compute derivatives of integrals over $\mathcal{S}_{u, v}$ with respect to the advanced and retarded null coordinates. The key observation in this respect is the following:

Lemma 7 (Computing derivatives of integrals over $\mathcal{S}_{u, v}$ ) Given a scalar $\phi$ one has that

$$
\begin{aligned}
\frac{\mathrm{d}}{\mathrm{d} v} \int_{\mathcal{S}_{u, v}} \phi & =\int_{\mathcal{S}_{u, v}}(D \phi-2 \rho \phi), \\
\frac{\mathrm{d}}{\mathrm{d} u} \int_{\mathcal{S}_{u, v}} \phi & =\int_{\mathcal{S}_{u, v}} Q^{-1}(\Delta \phi+2 \mu \phi),
\end{aligned}
$$

along the outgoing and incoming null geodesics that rule $\mathcal{N}_{v}$ and $\mathcal{N}_{u}$.

Proof The proof follows a direct computation. More precisely, one has that

$$
\begin{aligned}
\frac{\mathrm{d}}{\mathrm{d} v} \int_{\mathcal{S}_{u, v}} \phi & =\int_{\mathcal{S}_{u, v}} \frac{\partial}{\partial v}(\phi \sqrt{|\operatorname{det} \sigma|}) \mathrm{d} x^{2} \mathrm{~d} x^{3} \\
& =\int_{\mathcal{S}_{u, v}}\left(D(\phi \sqrt{|\operatorname{det} \sigma|})-C^{\mathcal{A}} \partial_{\mathcal{A}}(\phi \sqrt{\operatorname{det} \sigma})\right) \mathrm{d} x^{2} \mathrm{~d} x^{3}
\end{aligned}
$$




$$
=\int_{\mathcal{S}_{u, v}}\left(D \phi \sqrt{|\operatorname{det} \sigma|}+\phi D \sqrt{|\operatorname{det} \sigma|}-C^{\mathcal{A}} \partial_{\mathcal{A}}(\phi \sqrt{|\operatorname{det} \sigma|})\right) \mathrm{d} x^{2} \mathrm{~d} x^{3} .
$$

For the second term in the integrand, $\phi D \sqrt{|\operatorname{det} \sigma|}$, we find that

$$
\begin{aligned}
D \sqrt{|\operatorname{det} \sigma|} & =\frac{1}{2 \sqrt{|\operatorname{det} \sigma|}} D \operatorname{det} \sigma=\frac{|\operatorname{det} \sigma|}{2 \sqrt{|\operatorname{det} \sigma|}} \sigma^{\mathcal{A B}} D \sigma_{\mathcal{A B}}=-\frac{\sqrt{|\operatorname{det} \sigma|}}{2} \sigma_{\mathcal{A B}} D \sigma^{\mathcal{A B}} \\
& =\sqrt{|\operatorname{det} \sigma|} \sigma_{\mathcal{A B}}\left(\bar{P}^{\mathcal{B}} D P^{\mathcal{A}}+P^{\mathcal{A}} D \bar{P}^{\mathcal{B}}\right) \\
& =\sqrt{|\operatorname{det} \sigma|}\left(\sigma_{\mathcal{A B}} \bar{P}^{\mathcal{B}} \delta C^{\mathcal{A}}+\sigma_{\mathcal{A B}} P^{\mathcal{A}} \bar{\delta} C^{\mathcal{B}}-2 \rho+\sigma \sigma_{\mathcal{A B}} \bar{P}^{\mathcal{A}} \bar{P}^{\mathcal{B}}+\bar{\sigma} \sigma_{\mathcal{A B}} P^{\mathcal{A}} P^{\mathcal{B}}\right) \\
& =\sqrt{|\operatorname{det} \sigma|}\left(\bar{m}_{\mathcal{A}} \delta C^{\mathcal{A}}+m_{\mathcal{A}} \bar{\delta} C^{\mathcal{A}}-2 \rho\right)=-\sqrt{|\operatorname{det} \sigma|}\left(\partial_{\mathcal{A}} C^{\mathcal{A}}+2 \rho\right),
\end{aligned}
$$

where we have used Remark 2 and the structure Eq. (12c). For the third term in the integral one has that

$$
\begin{aligned}
& \int_{\mathcal{S}_{u, v} C^{\mathcal{A}} \partial_{\mathcal{A}}(\phi \sqrt{|\operatorname{det} \sigma|}) \mathrm{d} x^{2} \mathrm{~d} x^{3}=} \int_{\mathcal{S}_{u, v}} \partial_{\mathcal{A}}\left(C^{\mathcal{A}} \phi \sqrt{|\operatorname{det} \sigma|}\right) \mathrm{d} x^{2} \mathrm{~d} x^{3} \\
&-\int_{\mathcal{S}_{u, v}} \phi \partial_{\mathcal{A}} C^{\mathcal{A}} \sqrt{|\operatorname{det} \sigma|} \mathrm{d} x^{2} \mathrm{~d} x^{3} \\
&=-\int_{\mathcal{S}_{u, v}} \phi \partial_{\mathcal{A}} C^{\mathcal{A}} \sqrt{|\operatorname{det} \sigma|} \mathrm{d} x^{2} \mathrm{~d} x^{3} \\
&+\int_{\mathcal{S}_{u, v}} \nabla_{\mathcal{A}}\left(C^{\mathcal{A}} \phi \sqrt{|\operatorname{det} \sigma|}\right) \mathrm{d} x^{2} \mathrm{~d} x^{3} \\
&=-\int_{\mathcal{S}_{u, v}} \phi \partial_{\mathcal{A}} C^{\mathcal{A}} \sqrt{|\operatorname{det} \sigma|} \mathrm{d} x^{2} \mathrm{~d} x^{3},
\end{aligned}
$$

where for the last equality we have use Stokes' theorem and the fact that sphere has no boundary. Combining the above observations one finds that

$$
\frac{\mathrm{d}}{\mathrm{d} v} \int_{\mathcal{S}_{u, v}} \phi=\int_{\mathcal{S}_{u, v}}(D \phi-2 \rho \phi) \sqrt{|\operatorname{det} \sigma|} \mathrm{d} x^{2} \mathrm{~d} x^{3} .
$$

To compute the derivative with respect to $u$, we first consider

$$
\begin{aligned}
& \Delta \sqrt{|\operatorname{det} \sigma|}=-\frac{1}{2} \sqrt{|\operatorname{det} \sigma|} \sigma_{\mathcal{A B}} \Delta \sigma^{\mathcal{A B}}=\frac{1}{2} \sqrt{|\operatorname{det} \sigma|} \sigma_{\mathcal{A B}}\left(\bar{P}^{\mathcal{B}} \Delta P^{\mathcal{A}}+P^{\mathcal{A}} \Delta \bar{P}^{\mathcal{B}}\right) \\
& =\frac{1}{2} \sqrt{|\operatorname{det} \sigma|} \sigma_{\mathcal{A B}}\left(\bar{P}^{\mathcal{B}}\left(-\mu P^{\mathcal{A}}-\bar{\lambda} \bar{P}^{\mathcal{A}}\right)+P^{\mathcal{A}}\left(-\bar{\mu} \bar{P}^{\mathcal{B}}-\lambda P^{\mathcal{B}}\right)\right) \\
& =\frac{1}{2}(\mu+\bar{\mu}) \sqrt{|\operatorname{det} \sigma|}=\mu \sqrt{|\operatorname{det} \sigma|} \text {. }
\end{aligned}
$$

From the above identity one readily obtains

$$
\frac{\mathrm{d}}{\mathrm{d} u} \int_{\mathcal{S}_{u, v}} \phi=\int_{\mathcal{S}_{u, v}} \frac{\partial}{\partial u}(\phi \sqrt{|\operatorname{det} \sigma|}) \mathrm{d} x^{2} \mathrm{~d} x^{3}
$$




$$
\begin{aligned}
& =\int_{\mathcal{S}_{u, v}} Q^{-1}(\sqrt{|\operatorname{det} \sigma|} \Delta \phi+\phi \Delta \sqrt{|\operatorname{det} \sigma|}) \mathrm{d} x^{2} \mathrm{~d} x^{3} \\
& =\int_{\mathcal{S}_{u, v}} Q^{-1}(\Delta \phi+2 \mu \phi) \sqrt{|\operatorname{det} \sigma|} \mathrm{d} x^{2} \mathrm{~d} x^{3}
\end{aligned}
$$

as required.

Integrals over $\mathcal{D}_{u, v}$. The construction of energy-type estimates for the components of the Weyl tensor require further integral identities. These integrals allow us to write the integral over the diamond $\mathcal{D}_{u, v}$ of the $D$ and $\Delta$-derivatives of the components of the Weyl tensor in terms of integrals on the light cones and an integral over the bulk diamond of the (undifferentiated) components.

Lemma 8 (Integral over causal diamonds of derivatives of a scalar) Let $f$ be a scalar field in the causal diamond $\mathcal{D}_{u, v}$. One has then that

$$
\begin{aligned}
& \int_{\mathcal{D}_{u, v}} D f=\int_{\mathcal{N}_{v}(0, u)} Q^{-1} f-\int_{\mathcal{N}_{0}(0, u)} Q^{-1} f+\int_{\mathcal{D}_{u, v}}(2 \rho+\epsilon+\bar{\epsilon}) f, \\
& \int_{\mathcal{D}_{u, v}} \Delta f=\int_{\mathcal{N}_{u}(0, v)} f-\int_{\mathcal{N}_{0}(0, v)} f-\int_{\mathcal{D}_{u, v}} 2 \mu f .
\end{aligned}
$$

Proof The proof of the identities follows by integration by parts. For the long direction we have, by definition, that

$$
\int_{\mathcal{D}_{u, v}} D f=\int_{0}^{u} \int_{0}^{v} \int_{\mathcal{S}_{u^{\prime}, v^{\prime}}} Q^{-1}\left(\partial_{v} f+C^{\mathcal{A}} \partial_{\mathcal{A}} f\right) \sqrt{|\operatorname{det} \sigma|} \mathrm{d} x^{2} \mathrm{~d} x^{3} \mathrm{~d} u^{\prime} \mathrm{d} v^{\prime} .
$$

Now, on the one hand, integrating by parts with respect to $v$ one has that,

$$
\begin{aligned}
\int_{0}^{u} & \int_{0}^{v} \int_{\mathcal{S}_{u^{\prime}, v^{\prime}}} Q^{-1} \partial_{v} f \sqrt{|\operatorname{det} \sigma|} \mathrm{d} x^{2} \mathrm{~d} x^{3} \mathrm{~d} v^{\prime} \mathrm{d} u^{\prime} \\
= & \int_{0}^{u} \int_{0}^{v} \int_{\mathcal{S}_{u^{\prime}, v^{\prime}}} \partial_{v}\left(Q^{-1} f \sqrt{|\operatorname{det} \sigma|}\right) \mathrm{d} x^{2} \mathrm{~d} x^{3} \mathrm{~d} v^{\prime} \mathrm{d} u^{\prime} \\
& -\int_{0}^{u} \int_{0}^{v} \int_{\mathcal{S}_{u^{\prime}, v^{\prime}}} f \partial_{v}\left(Q^{-1} \sqrt{|\operatorname{det} \sigma|}\right) \mathrm{d} x^{2} \mathrm{~d} x^{3} \mathrm{~d} v^{\prime} \mathrm{d} u^{\prime}, \\
= & \int_{\mathcal{N}_{v}(0, u)} Q^{-1} f-\int_{\mathcal{N}_{0}(0, u)} Q^{-1} f \\
& -\int_{0}^{u} \int_{0}^{v} \int_{\mathcal{S}_{u^{\prime}, v^{\prime}}}\left(f \partial_{v} Q^{-1} \sqrt{|\operatorname{det} \sigma|}+Q^{-1} f \partial_{v} \sqrt{|\operatorname{det} \sigma|}\right) \mathrm{d} x^{2} \mathrm{~d} x^{3} \mathrm{~d} v^{\prime} \mathrm{d} u^{\prime} .
\end{aligned}
$$

On the other hand, integration by parts respect to the angular coordinates gives

$$
\int_{0}^{u} \int_{0}^{v} \int_{\mathcal{S}_{u^{\prime}, v^{\prime}}} Q^{-1} C^{\mathcal{A}} \partial_{\mathcal{A}} f \sqrt{|\operatorname{det} \sigma|} \mathrm{d} x^{2} \mathrm{~d} x^{3} \mathrm{~d} v^{\prime} \mathrm{d} u^{\prime}
$$




$$
\begin{aligned}
= & -\int_{0}^{u} \int_{0}^{v} \int_{\mathcal{S}_{u^{\prime}, v^{\prime}}} f \partial_{\mathcal{A}}\left(Q^{-1} C^{\mathcal{A}} \sqrt{|\operatorname{det} \sigma|}\right) \mathrm{d} x^{2} \mathrm{~d} x^{3} \mathrm{~d} v^{\prime} \mathrm{d} u^{\prime}, \\
= & -\int_{0}^{u} \int_{0}^{v} \int_{\mathcal{S}_{u^{\prime}, v^{\prime}}}\left(f \sqrt{|\operatorname{det} \sigma|} C^{\mathcal{A}} \partial_{\mathcal{A}} Q^{-1}+Q^{-1} f \sqrt{|\operatorname{det} \sigma|} \partial_{\mathcal{A}} C^{\mathcal{A}}\right. \\
& \left.+Q^{-1} f C^{\mathcal{A}} \partial_{\mathcal{A}} \sqrt{|\operatorname{det} \sigma|}\right) \mathrm{d} x^{2} \mathrm{~d} x^{3} \mathrm{~d} v^{\prime} \mathrm{d} u^{\prime} .
\end{aligned}
$$

Thus, we have

$$
\begin{aligned}
\int_{\mathcal{D}_{u, v}} D f= & \int_{\mathcal{N}_{v}(0, u)} Q^{-1} f-\int_{\mathcal{N}_{0}(0, u)} Q^{-1} f \\
& -\int_{0}^{u} \int_{0}^{v} \int_{S_{u^{\prime}, v^{\prime}}}\left(\sqrt{|\operatorname{det} \sigma|} f Q^{-2} D Q\right. \\
& \left.+Q^{-1} f D \sqrt{|\operatorname{det} \sigma|}+Q^{-1} f \sqrt{|\operatorname{det} \sigma|} \partial_{\mathcal{A}} C^{\mathcal{A}}\right) \mathrm{d} x^{2} \mathrm{~d} x^{3} \mathrm{~d} v^{\prime} \mathrm{d} u^{\prime} .
\end{aligned}
$$

Finally, making use of the expressions for $D Q$ from Eq. (12d) and $D \sqrt{|\operatorname{det} \sigma|}$ from Proposition 7, respectively, one obtains the desired identity.

To demonstrate the identity along the short direction one proceeds in a similar fashion.

Corollary 2 If $f=f_{1} f_{2}$, then

$$
\begin{aligned}
& \int_{\mathcal{D}_{u, v}} f_{1} D f_{2}+\int_{\mathcal{D}_{u, v}} f_{2} D f_{1}=\int_{\mathcal{N}_{v}(0, u)} Q^{-1} f_{1} f_{2}-\int_{\mathcal{N}_{0}(0, u)} Q^{-1} f_{1} f_{2} \\
& +\int_{\mathcal{D}_{u, v}}(2 \rho+\epsilon+\bar{\epsilon}) f_{1} f_{2}, \\
& \int_{\mathcal{D}_{u, v}} f_{1} \Delta f_{2}+\int_{\mathcal{D}_{u, v}} f_{2} \Delta f_{1}=\int_{\mathcal{N}_{u}(0, v)} f_{1} f_{2}-\int_{\mathcal{N}_{0}(0, v)} f_{1} f_{2}-\int_{\mathcal{D}_{u, v}} 2 \mu f_{1} f_{2} .
\end{aligned}
$$

Basic $L^{p}$ estimates The first step in the analysis is the construction of $L^{p}$ estimates. These estimates require a priori control of the NP spin connection coefficients $\rho$ and $\mu$. The reason for their special treatment can be traced back to their appearance in Lemma 7. Proceeding in this way we obtain the following:

Proposition 2 (Control of the $L^{p}$-norm with transport equations) Work under Assumption 1. Assume furthermore on $\mathcal{D}_{u, v_{\bullet}}^{t}$ that

$$
\sup _{u, v}\|\{\rho, \mu\}\|_{L^{\infty}\left(\mathcal{S}_{u, v}\right)} \leq \mathcal{O}
$$

Then there exists $\varepsilon_{\star}=\varepsilon_{\star}\left(\Delta_{e_{\star}}, \mathcal{O}\right)$ such that for all $\varepsilon \leq \varepsilon_{\star}$ and for every $1 \leq p<\infty$, we have the estimates:

$$
\|\phi\|_{L^{p}\left(\mathcal{S}_{u, v}\right)} \leq C(I, \mathcal{O})\left(\|\phi\|_{L^{p}\left(\mathcal{S}_{u, 0}\right)}+\int_{0}^{v}\|D \phi\|_{L^{p}\left(\mathcal{S}_{u, v^{\prime}}\right)} \mathrm{d} v^{\prime}\right),
$$




$$
\|\phi\|_{L^{p}\left(\mathcal{S}_{u, v}\right)} \leq 2\left(\|\phi\|_{L^{p}\left(\mathcal{S}_{0, v}\right)}+C\left(\Delta_{e_{\star}}, \mathcal{O}\right) \int_{0}^{u}\|\Delta \phi\|_{L^{p}\left(\mathcal{S}_{u^{\prime}, v}\right)} \mathrm{d} u^{\prime}\right)
$$

where, as elsewhere, I denotes the long direction interval.

Proof Making use of the definition of $\|\phi\|_{L^{p}\left(\mathcal{S}_{u, v}\right)}$ and the identity in Lemma 7, we have

$$
\begin{aligned}
\|\phi\|_{L^{p}\left(\mathcal{S}_{u, v}\right)}^{p} & =\|\phi\|_{L^{p}\left(\mathcal{S}_{u, 0}\right)}^{p}+\int_{0}^{v} \frac{\mathrm{d}}{\mathrm{d} v}\|\phi\|_{L^{p}\left(\mathcal{S}_{u, v^{\prime}}\right)}^{p} \mathrm{~d} v^{\prime} \\
& =\|\phi\|_{L^{p}\left(\mathcal{S}_{u, 0}\right)}^{p}+\int_{0}^{v}\left(\frac{\mathrm{d}}{\mathrm{d} v} \int_{\mathcal{S}_{u, v^{\prime}}}|\phi|^{p}\right) \mathrm{d} v^{\prime} \\
& =\|\phi\|_{L^{p}\left(\mathcal{S}_{u, 0}\right)}^{p}+\int_{0}^{v}\left(\int_{\mathcal{S}_{u, v^{\prime}}}\left(D|\phi|^{p}-2 \rho|\phi|^{p}\right)\right) \mathrm{d} v^{\prime} .
\end{aligned}
$$

Now, Young's inequality gives

$D|\phi|^{p}=p|\phi|^{p-1} D|\phi| \leq p\left(\frac{\left(|\phi|^{p-1}\right)^{\frac{p}{p-1}}}{p /(p-1)}+\frac{(D|\phi|)^{p}}{p}\right)=(p-1)|\phi|^{p}+(D|\phi|)^{p}$.

Thus, we have that

$$
\begin{aligned}
\|\phi\|_{L^{p}\left(\mathcal{S}_{u, v}\right)}^{p} & \leq\|\phi\|_{L^{p}\left(\mathcal{S}_{u, 0}\right)}^{p}+\int_{0}^{v}\left(\int_{\mathcal{S}_{u, v^{\prime}}}(D|\phi|)^{p}+(p-1-2 \rho)|\phi|^{p}\right) \mathrm{d} v^{\prime} \\
& \leq\|\phi\|_{L^{p}\left(\mathcal{S}_{u, 0}\right)}^{p}+\int_{0}^{v}\left(\int_{\mathcal{S}_{u, v^{\prime}}}(D|\phi|)^{p}+C_{1}(\mathcal{O})|\phi|^{p}\right) \mathrm{d} v^{\prime} \\
& \leq\|\phi\|_{L^{p}\left(\mathcal{S}_{u, 0}\right)}^{p}+\int_{0}^{v}\|D \phi\|_{L^{p}\left(\mathcal{S}_{u, v^{\prime}}\right)}^{p} \mathrm{~d} v^{\prime}+C_{1}(\mathcal{O}) \int_{0}^{v}\|\phi\|_{L^{p}\left(\mathcal{S}_{u, v^{\prime}}\right)}^{p} \mathrm{~d} v^{\prime} .
\end{aligned}
$$

Now, making use of Grönwall's inequality, we obtain

$$
\begin{aligned}
\|\phi\|_{L^{p}\left(\mathcal{S}_{u, v}\right)}^{p} & \leq C(I, \mathcal{O})\left(\|\phi\|_{L^{p}\left(\mathcal{S}_{u, 0}\right)}^{p}+\int_{0}^{v}\|D \phi\|_{L^{p}\left(\mathcal{S}_{u, v^{\prime}}\right)}^{p} \mathrm{~d} v^{\prime}\right) \\
& \leq C(I, \mathcal{O})\left(\|\phi\|_{L^{p}\left(\mathcal{S}_{u, 0}\right)}^{p}+\left(\int_{0}^{v}\|D \phi\|_{L^{p}\left(\mathcal{S}_{u, v^{\prime}}\right)} \mathrm{d} v^{\prime}\right)^{p}\right) \\
& \leq C(I, \mathcal{O})\left(\|\phi\|_{L^{p}\left(\mathcal{S}_{u, 0}\right)}+\int_{0}^{v}\|D \phi\|_{L^{p}\left(\mathcal{S}_{u, v^{\prime}}\right)} \mathrm{d} v^{\prime}\right)^{p},
\end{aligned}
$$

so that, in fact, one has

$$
\|\phi\|_{L^{p}\left(\mathcal{S}_{u, v}\right)} \leq C(I, \mathcal{O})\left(\|\phi\|_{L^{p}\left(\mathcal{S}_{u, 0}\right)}+\int_{0}^{v}\|D \phi\|_{L^{p}\left(\mathcal{S}_{u, v^{\prime}}\right)} \mathrm{d} v^{\prime}\right) .
$$


Now, for the integration in the short direction $0 \leq u \leq \varepsilon$, using the assumption that $\sup _{u, v}\|\mu\|_{L^{\infty}\left(\mathcal{S}_{u, v}\right)} \leq \mathcal{O}$, a similar argument as before, and now using Lemma 5, allows us to show that

$$
\begin{aligned}
\|\phi\|_{L^{p}\left(\mathcal{S}_{u, v}\right)}^{p} & \|\phi\|_{L^{p}\left(\mathcal{S}_{0, v}\right)}^{p}+C\left(\Delta_{e_{\star}}\right) \\
& \left(C(\mathcal{O}) \int_{0}^{u}\|\phi\|_{L^{p}\left(\mathcal{S}_{u^{\prime}, v}\right)}^{p} \mathrm{~d} u^{\prime}+\int_{0}^{u}\|\Delta \phi\|_{L^{p}\left(\mathcal{S}_{u^{\prime}, v}\right)}^{p} \mathrm{~d} u^{\prime}\right),
\end{aligned}
$$

so that one has

$$
\begin{aligned}
\|\phi\|_{L^{p}\left(\mathcal{S}_{u, v}\right) \leq} & \|\phi\|_{L^{p}\left(\mathcal{S}_{0, v}\right)}+C\left(\Delta_{e_{\star}}, \mathcal{O}\right) \\
& \left(\int_{0}^{u}\|\phi\|_{L^{p}\left(\mathcal{S}_{u^{\prime}, v}\right)} \mathrm{d} u^{\prime}+\int_{0}^{u}\|\Delta \phi\|_{L^{p}\left(\mathcal{S}_{u^{\prime}, v}\right)} \mathrm{d} u^{\prime}\right) .
\end{aligned}
$$

Then, using Grönwall's inequality one is led to

$$
\|\phi\|_{L^{p}\left(\mathcal{S}_{u, v}\right)} \leq \exp \left(C\left(\Delta_{e_{\star}}, \mathcal{O}\right) \varepsilon\right)\left(\|\phi\|_{L^{p}\left(\mathcal{S}_{0, v}\right)}+C\left(\Delta_{e_{\star}}, \mathcal{O}\right) \int_{0}^{u}\|\Delta \phi\|_{L^{p}\left(\mathcal{S}_{u^{\prime}, v}\right)} \mathrm{d} u^{\prime}\right)
$$

From, the latter choosing $\varepsilon>0$ small enough one concludes that

$$
\|\phi\|_{L^{p}\left(\mathcal{S}_{u, v}\right)} \leq 2\left(\|\phi\|_{L^{p}\left(\mathcal{S}_{0, v}\right)}+C\left(\Delta_{e_{\star}}, \mathcal{O}\right) \int_{0}^{u}\|\Delta \phi\|_{L^{p}\left(\mathcal{S}_{u^{\prime}, v}\right)} \mathrm{d} u^{\prime}\right)
$$

As a particular example of the previous discussion consider $\phi=\delta f$, with $p=2$. In this case one has

$$
\|\delta f\|_{L^{2}\left(\mathcal{S}_{u, v}\right)} \leq C(I, \mathcal{O})\left(\|\delta f\|_{L^{2}\left(\mathcal{S}_{u, 0}\right)}+\int_{0}^{v}\left(\int_{\mathcal{S}_{u, v^{\prime}}} D|\delta f|^{2}\right)^{1 / 2} \mathrm{~d} v^{\prime}\right) .
$$

If $p=4$ one has that

$$
\|\delta f\|_{L^{4}\left(\mathcal{S}_{u, v}\right)} \leq C(I, \mathcal{O})\left(\|\delta f\|_{L^{4}\left(\mathcal{S}_{u, 0}\right)}+\int_{0}^{v}\left(\int_{\mathcal{S}_{u, v^{\prime}}} D|\delta f|^{2}\right)^{1 / 4} \mathrm{~d} v^{\prime}\right) .
$$

For the short direction one readily obtains analogous expressions.

Basic $L^{\infty}$ estimates Our analysis will also require estimates on the $L^{\infty}$ norm of various scalars. The first result in this direction is the following:

Proposition 3 (Supremum norm of solutions to transport equations) Work under Assumption 1. There exists $\varepsilon_{\star}$ such that for all $\varepsilon \leq \varepsilon_{\star}$, we have 


$$
\begin{aligned}
& \|\phi\|_{L^{\infty}\left(\mathcal{S}_{u, v}\right)} \leq\|\phi\|_{L^{\infty}\left(\mathcal{S}_{u, 0}\right)}+\int_{0}^{v}\|D \phi\|_{L^{\infty}\left(\mathcal{S}_{u, v^{\prime}}\right)} \mathrm{d} v^{\prime}, \\
& \|\phi\|_{L^{\infty}\left(\mathcal{S}_{u, v}\right)} \leq\|\phi\|_{L^{\infty}\left(\mathcal{S}_{0, v}\right)}+C\left(\Delta_{e_{\star}}\right) \int_{0}^{u}\|\Delta \phi\|_{L^{\infty}\left(\mathcal{S}_{u^{\prime}, v}\right)} \mathrm{d} u^{\prime},
\end{aligned}
$$

on $\mathcal{D}_{u, v_{\bullet}}^{t}$.

Proof Given a fixed point $\left(u, 0, x^{\mathcal{A}}\right)$ on $\mathcal{N}_{\star}$, and then integrating out along integral curves of $l^{a}$, conveniently parametrizing with $v$, gives

$$
\phi_{\mathcal{S}_{u, v}}-\phi_{\mathcal{S}_{u, 0}}=\int_{0}^{v} \frac{d \phi}{d v} \mathrm{~d} v^{\prime}=\int_{0}^{v} D \phi \mathrm{d} v^{\prime}
$$

Fixing $u$, varying the angular point $x^{A}$ on $\mathcal{N}_{\star}$ arbitrarily, and taking the supremum we obtain the inequality of the of the proposition. The proof of the second inequality is similar.

More advanced $L^{p}$-estimates Finally, we discuss the construction of more refined $L^{p}$-estimates. As in the case of the basic $L^{p}$-estimates, these estimates require some a priori control on the $L^{\infty}$-norm of the the NP spin connection coefficients $\rho$ and $\mu$. More precisely, one has the following:

Proposition 4 ( $L^{4}$-norm of solutions to transport equations) Work under Assumption 1. Assume, as in Proposition 2, furthermore that

$$
\sup _{u, v}\|\{\rho, \mu\}\|_{L^{\infty}\left(\mathcal{S}_{u, v}\right)} \leq \mathcal{O} \text {. }
$$

on $\mathcal{D}_{u, v_{\bullet}}^{t}$. Then there exists $\varepsilon_{\star}=\varepsilon_{\star}\left(\Delta_{e_{\star}}, \mathcal{O}\right)$ such that for all $\varepsilon \leq \varepsilon_{\star}$ we have the estimates:

$$
\begin{gathered}
\|\phi\|_{L^{4}\left(\mathcal{S}_{u, v}\right)} \leq C\left(\Delta_{e_{\star}}, \mathcal{O}\right)\left(\|\phi\|_{L^{4}\left(\mathcal{S}_{u, 0}\right)}+\|D \phi\|_{L^{2}\left(\mathcal{N}_{u}(0, v)\right)}^{1 / 2}\right. \\
\left.\left(\|\phi\|_{L^{2}\left(\mathcal{N}_{u}(0, v)\right)}^{2}+\|\not \nabla \phi\|_{L^{2}\left(\mathcal{N}_{u}(0, v)\right)}^{2}\right)^{1 / 4}\right) \\
\|\phi\|_{L^{4}\left(\mathcal{S}_{u, v}\right)} \leq 2\left(\|\phi\|_{L^{4}\left(\mathcal{S}_{0, v}\right)}+C\left(\Delta_{e_{\star}}\right)\|\Delta \phi\|_{L^{2}\left(\mathcal{N}_{v}(0, u)\right)}^{1 / 2}\right. \\
\left.\left(\|\phi\|_{L^{2}\left(\mathcal{N}_{v}(0, u)\right)}^{2}+\|\not \nabla \phi\|_{L^{2}\left(\mathcal{N}_{v}(0, u)\right)}^{2}\right)^{1 / 4}\right)
\end{gathered}
$$

on $\mathcal{D}_{u, v_{\bullet}}^{t}$.

Proof The proof proceeds by direct computation. We first obtain the estimate on the long direction. Following arguments similar to those used in Proposition 2, we find that

$$
\|\phi\|_{L^{4}\left(\mathcal{S}_{u, v}\right)}^{4}=\|\phi\|_{L^{4}\left(\mathcal{S}_{u, 0}\right)}^{4}+\int_{0}^{v}\left(\int_{S_{u, v^{\prime}}} D|\phi|^{4}-2 \rho|\phi|^{4}\right) \mathrm{d} v^{\prime}
$$




$$
\begin{aligned}
\leq & \|\phi\|_{L^{4}\left(\mathcal{S}_{u, 0}\right)}^{4}+2 \mathcal{O} \int_{0}^{v}\|\phi\|_{L^{4}\left(\mathcal{S}_{u, v^{\prime}}\right)}^{4} \mathrm{~d} v^{\prime} \\
& +4\left(\int_{\mathcal{N}_{u}(0, v)}|\phi|^{6}\right)^{1 / 2}\left(\int_{\mathcal{N}_{u}(0, v)}|D \phi|^{2}\right)^{1 / 2} .
\end{aligned}
$$

Now, for small enough $\varepsilon$, using the Nirenberg-Sobolev inequality (see "Appendix B") we estimate:

$$
\begin{aligned}
\int_{\mathcal{N}_{u}(0, v)}|\phi|^{6} & =\int_{0}^{v} \int_{\mathcal{S}_{u, v^{\prime}}}|\phi|^{6} \mathrm{~d} v^{\prime}=\int_{0}^{v}\left\||\phi|^{3}\right\|_{L^{2}\left(\mathcal{S}_{u, v^{\prime}}\right)}^{2} \mathrm{~d} v^{\prime} \\
& \leq C\left(\Delta_{e_{\star}}\right) \int_{0}^{v}\left(\left\||\phi|^{3}\right\|_{L^{1}\left(\mathcal{S}_{u, v^{\prime}}\right)}+\left\|\left.\not|| \phi\right|^{3}\right\|_{L^{1}\left(\mathcal{S}_{u, v^{\prime}}\right)}\right)^{2} \mathrm{~d} v^{\prime} \\
& \leq C\left(\Delta_{e_{\star}}\right) \int_{0}^{v}\left(\left\||\phi|^{2}\right\|_{L^{2}\left(\mathcal{S}_{u, v^{\prime}}\right)}\|\phi\|_{L^{2}\left(\mathcal{S}_{u, v^{\prime}}\right)}+\left\|\left.\phi\right|^{2}\right\|_{L^{2}\left(\mathcal{S}_{u, v^{\prime}}\right)}\|\not \nabla \phi\|_{L^{2}\left(\mathcal{S}_{u, v^{\prime}}\right)}\right)^{2} \mathrm{~d} v^{\prime} \\
& \leq C\left(\Delta_{e_{\star}}\right) \int_{0}^{v}\|\phi\|_{L^{4}\left(\mathcal{S}_{u, v^{\prime}}\right)}^{4}\left(\|\phi\|_{L^{2}\left(\mathcal{S}_{u, v^{\prime}}\right)}+\|\not \nabla \phi\|_{L^{2}\left(\mathcal{S}_{u, v^{\prime}}\right)^{2} \mathrm{~d} v^{\prime}}\right. \\
& \leq 2 C\left(\Delta_{\left.e_{\star}\right)}\left(\sup _{u, v}\|\phi\|_{L^{4}\left(\mathcal{S}_{u, v}\right)}^{4}\right) \int_{0}^{v}\left(\|\phi\|_{L^{2}\left(\mathcal{S}_{u, v^{\prime}}\right)}^{2}+\|\not \nabla \phi\|_{L^{2}\left(\mathcal{S}_{u, v^{\prime}}\right)}^{2}\right) \mathrm{d} v^{\prime}\right. \\
& \leq C\left(\Delta_{\left.e_{\star}\right)}\left(\sup _{u, v}\|\phi\|_{L^{4}\left(\mathcal{S}_{u, v}\right)}^{4}\right)\left(\|\phi\|_{L^{2}\left(\mathcal{N}_{u}(0, v)\right)}^{2}+\|\not \nabla \phi\|_{L^{2}\left(\mathcal{N}_{u}(0, v)\right)}^{2}\right)\right.
\end{aligned}
$$

where to pass from the second to the third line we have made use of Hölder's inequality and, to pass from the third to fourth we have extracted common factors. Making use of the above estimate in inequality (18), we have that

$$
\begin{aligned}
\|\phi\|_{L^{4}\left(\mathcal{S}_{u, v}\right)}^{4} \leq & \|\phi\|_{L^{4}\left(\mathcal{S}_{u, 0}\right)}^{4}+2 \mathcal{O} \int_{0}^{v}\|\phi\|_{L^{4}\left(\mathcal{S}_{u, v^{\prime}}\right)}^{4} \mathrm{~d} v^{\prime} \\
& +C\left(\Delta_{e_{\star}}\right)\left(\sup _{u, v}\|\phi\|_{L^{4}\left(\mathcal{S}_{u, v}\right)}^{2}\right)\left(\|\phi\|_{L^{2}\left(\mathcal{N}_{u}(0, v)\right)}^{2}+\|\not \nabla \phi\|_{L^{2}\left(\mathcal{N}_{u}(0, v)\right)}^{2}\right)^{1 / 2} \\
& \|D \phi\|_{L^{2}\left(\mathcal{N}_{u}(0, v)\right)} \\
\leq & \|\phi\|_{L^{4}\left(\mathcal{S}_{u, 0}\right)}^{4}+2 \mathcal{O} \int_{0}^{v}\|\phi\|_{L^{4}\left(\mathcal{S}_{u, v^{\prime}}\right)}^{4} \mathrm{~d} v^{\prime}+C\left(\Delta_{e_{\star}}\right) \delta\left(\sup _{u, v}\|\phi\|_{L^{4}\left(\mathcal{S}_{u, v}\right)}^{4}\right) \\
& +\frac{C\left(\Delta_{e_{\star}}\right)}{4 \delta}\left(\|\phi\|_{L^{2}\left(\mathcal{N}_{u}(0, v)\right)}^{2}+\|\not \nabla \phi\|_{L^{2}\left(\mathcal{N}_{u}(0, v)\right)}^{2}\right)\|D \phi\|_{L^{2}\left(\mathcal{N}_{u}(0, v)\right)}^{2},
\end{aligned}
$$

for some $\delta>0$. Now, choosing $\delta$ sufficiently small and making use of Grönwall's inequality, one finally obtains that

$$
\begin{aligned}
\|\phi\|_{L^{4}\left(\mathcal{S}_{u, v}\right)}^{4} \leq & C\left(\Delta_{e_{\star}}, \mathcal{O}\right)\left(\|\phi\|_{L^{4}\left(\mathcal{S}_{u, 0}\right)}^{4}+\|D \phi\|_{L^{2}\left(\mathcal{N}_{u}(0, v)\right)}^{2}\right. \\
& \left.\left(\|\phi\|_{L^{2}\left(\mathcal{N}_{u}(0, v)\right)}^{2}+\|\not \phi \phi\|_{L^{2}\left(\mathcal{N}_{u}(0, v)\right)}^{2}\right)\right)
\end{aligned}
$$

The proof of the estimate along the short direction is similar. In this case we can choose $\varepsilon>0$ sufficiently small to make the overall constant equal to, say, 2 . 


\subsection{Sobolev inequalities}

In the last step in our preparatory work, we now obtain Sobolev-type inequalities on the spheres $\mathcal{S}_{u, v}$-i.e. estimates of the $L^{p}$-norms of a scalar in terms of its $L^{2}$-norms and those of its derivatives. The key tool in this analysis is the isoperimetric Sobolev inequality on $\mathcal{S}_{u, v}$ - see [16]:

Theorem 3 (Isoperimetric Sobolev inequality on $\mathcal{S}_{u, v}$ ) Let $\phi$ denote an integrable function and with integrable first derivatives on $\mathcal{S}_{u, v}$. Then we have that

$$
\int_{\mathcal{S}_{u, v}}|\phi-\bar{\phi}|^{2} \leq \mathcal{I}\left(\mathcal{S}_{u, v}\right)\left(\int_{\mathcal{S}_{u, v}}|\nabla \phi|\right)^{2},
$$

where $\bar{\phi}$ denotes the average of $\phi$ over $\mathcal{S}_{u, v}$ and $\mathcal{I}\left(\mathcal{S}_{u, v}\right)$ is the isoperimetric constant.

Remark 10 The isoperimetric inequality can be shown to be controlled by the area of the 2-dimensional surfaces $\mathcal{S}_{u, v}$-see e.g. [16]. Thus, if one has control over the area of the surface (as it is, in principle, in our setup), one has also control over the isoperimetric constant.

Using this we can prove the following result concerning Sobolev-type inequalities:

Proposition 5 (Sobolev-type inequality. I) Work under Assumption 1. Let $\phi$ be a scalar field on $\mathcal{S}_{u, v}$ which is square-integrable with square-integrable first covariant derivatives. Then for each $2<p<\infty, \phi \in L^{p}\left(\mathcal{S}_{u, v}\right)$, there exists $\varepsilon_{\star}=\varepsilon_{\star}\left(\Delta_{e_{\star}}, \Delta_{\Gamma}\right)$ such that as long as $\varepsilon \leq \varepsilon_{\star}$, we have

$$
\|\phi\|_{L^{p}\left(\mathcal{S}_{u, v}\right)} \leq G_{p}(\sigma)\left(\|\phi\|_{L^{2}\left(\mathcal{S}_{u, v}\right)}+\|\not \nabla \phi\|_{L^{2}\left(\mathcal{S}_{u, v}\right)}\right)
$$

where $G_{p}(\sigma)$ is a constant also depends on the isoperimetric constant $\mathcal{I}\left(\mathcal{S}_{u, v}\right)$ and $p$, but is controlled by some $C\left(\Delta_{e_{\star}}\right), \nabla$ is the induced connection on $\mathcal{S}_{u, v}$ which is associated with the metric $\sigma$.

Proof We make use of the following result which can be found in Lemma 5.1 in Chapter 5.2 of [17]:

$$
\begin{aligned}
& \left(\operatorname{Area}\left(\mathcal{S}_{u, v}\right)\right)^{-1 / p}\|\phi\|_{L^{p}\left(\mathcal{S}_{u, v}\right)} \leq C_{p} \sqrt{\mathcal{I}^{\prime}\left(\mathcal{S}_{u, v}\right)} \\
& \left(\left(\operatorname{Area}\left(\mathcal{S}_{u, v}\right)\right)^{-1 / 2}\|\phi\|_{L^{2}\left(\mathcal{S}_{u, v}\right)}+\|\not \nabla \phi\|_{L^{2}\left(\mathcal{S}_{u, v}\right)}\right)
\end{aligned}
$$

where $C_{p}$ is a numerical constant depending only on $p$,

$$
\mathcal{I}^{\prime}\left(\mathcal{S}_{u, v}\right)=\max \left\{1, \mathcal{I}\left(\mathcal{S}_{u, v}\right)\right\}
$$

where as above $\mathcal{I}\left(\mathcal{S}_{u, v}\right)$ is the isoperimetric constant of $\mathcal{S}_{u, v}$. Now, under Assumption 1 we have that the area of $\mathcal{S}_{u, v}$ is finite in the tilted rectangle. Accordingly, inequality (20) can be adapted to our particular setting. 
Consequently we have the following two results:

Proposition 6 (Sobolev-type inequality. II) Work under Assumption 1. There exists $\varepsilon_{\star}=\varepsilon_{\star}\left(\Delta_{e_{\star}}, \Delta_{\Gamma}\right)$ such that as long as $\varepsilon \leq \varepsilon_{\star}$, we have

$$
\|\phi\|_{L^{\infty}\left(\mathcal{S}_{u, v}\right)} \leq G_{p}(\sigma)\left(\|\phi\|_{L^{p}\left(\mathcal{S}_{u, v}\right)}+\|\not \nabla \phi\|_{L^{p}\left(\mathcal{S}_{u, v}\right)}\right),
$$

with $2<p<\infty$ and $G_{p}(\sigma) \leq C\left(\Delta_{e_{\star}}\right)$ as above.

Corollary 3 (Sobolev-type inequality. III) Work under Assumption 1. There exists $\varepsilon_{\star}=$ $\varepsilon_{\star}\left(\Delta_{e_{\star}}, \Delta_{\Gamma}\right)$ such that as long as $\varepsilon \leq \varepsilon_{\star}$, we have

$$
\begin{aligned}
& \|\phi\|_{L^{4}\left(\mathcal{S}_{u, v}\right)} \leq G(\boldsymbol{\sigma})\left(\|\phi\|_{L^{2}\left(\mathcal{S}_{u, v}\right)}+\|\not \nabla \phi\|_{L^{2}\left(\mathcal{S}_{u, v}\right)}\right) \\
& \|\phi\|_{L^{\infty}\left(\mathcal{S}_{u, v}\right)} \leq G(\boldsymbol{\sigma})\left(\|\phi\|_{L^{2}\left(\mathcal{S}_{u, v}\right)}+\|\not \nabla \phi\|_{L^{2}\left(\mathcal{S}_{u, v}\right)}+\left\|\not \nabla^{2} \phi\right\|_{L^{2}\left(\mathcal{S}_{u, v}\right)}\right)
\end{aligned}
$$

again with $G(\sigma) \leq C\left(\Delta_{e_{\star}}\right)$.

\section{Main estimates}

In this section we provide a discussion of the construction of the main estimates required to obtain the improved existence result for the CIVP. The arguments rely heavily on the preparatory work carried out in the previous section.

\subsection{Norms for the initial data}

The boostrap argument requires assumptions on the size of the initial data. Following Luk [18], we define the following:

(i) Norm for the initial value of the connection coefficients, given by

$$
\begin{aligned}
\Delta_{\Gamma_{\star}} & \equiv \sup _{\mathcal{S}_{u, v} \subset \mathcal{N}_{\star}, \mathcal{N}_{\star}} \sup _{\Gamma \in\{\mu, \lambda, \rho, \sigma, \alpha, \beta, \tau, \epsilon\}} \\
\max & \left\{1,\|\Gamma\|_{L^{\infty}\left(\mathcal{S}_{u, v}\right)}, \sum_{i=0}^{1}\left\|\nabla^{i} \Gamma\right\|_{L^{4}\left(\mathcal{S}_{u, v}\right)}, \sum_{i=0}^{2}\left\|\nabla^{i} \Gamma\right\|_{L^{2}\left(\mathcal{S}_{u, v}\right)}\right\} .
\end{aligned}
$$

(ii) Norm for the initial value of the components of the Weyl tensor, given by

$$
\begin{aligned}
\Delta \Psi_{\star} & \equiv \sup _{\mathcal{S}_{u, v} \subset \mathcal{N}_{\star}, \mathcal{N}_{\star}} \Psi \in\left\{\Psi_{0}, \Psi_{1}, \Psi_{2}, \Psi_{3}, \Psi_{4}\right\} \\
& \max \left\{1, \sum_{i=0}^{1}\left\|\nabla^{i} \Psi\right\|_{L^{4}\left(\mathcal{S}_{u, v}\right)}, \sum_{i=0}^{2}\left\|\nabla^{i} \Psi\right\|_{L^{2}\left(\mathcal{S}_{u, v}\right)}\right\} \\
+ & \sum_{i=0}^{3} \sup _{\Psi \in\left\{\Psi_{0}, \Psi_{1}, \Psi_{2}, \Psi_{3}\right\}}\left\|\nabla^{i} \Psi\right\|_{L^{2}\left(\mathcal{N}_{\star}\right)}+\sup _{\Psi \in\left\{\Psi_{1}, \Psi_{2}, \Psi_{3}, \Psi_{4}\right\}}\left\|\nabla^{i} \Psi\right\|_{L^{2}\left(\mathcal{N}_{\star}^{\prime}\right)} .
\end{aligned}
$$


(iii) Norm for the components of the Weyl tensor at later null hypersurfaces, given by

$$
\Delta_{\Psi} \equiv \sum_{i=0}^{3} \sup _{\Psi \in\left\{\Psi_{0}, \Psi_{1}, \Psi_{2}, \Psi_{3}\right\}} \sup _{u}\left\|\nabla^{i} \Psi\right\|_{L^{2}\left(\mathcal{N}_{u}^{t}\right)}+\sup _{\Psi \in\left\{\Psi_{1}, \Psi_{2}, \Psi_{3}, \Psi_{4}\right\}} \sup _{v}\left\|\nabla^{i} \Psi\right\|_{L^{2}\left(\mathcal{N}_{v}^{t}\right)}
$$

where the suprema in $u$ and $v$ are taken over $\mathcal{D}_{u, v_{\bullet}}^{t}$.

(iv) Sup over the $L^{2}$-norm of the components of the Weyl tensor at spheres of constant $u, v$, given by,

$$
\Delta_{\Psi}(\mathcal{S})=\sum_{i=0}^{2} \sup _{u, v}\left\|\nabla^{i}\left(\Psi_{0}, \Psi_{1}, \Psi_{2}, \Psi_{3}\right)\right\|_{L^{2}\left(\mathcal{S}_{u, v}\right)}
$$

with the supremum taken over $\mathcal{D}_{u, v_{\bullet}}^{t}$, and in which $u$ will be taken sufficiently small to apply our estimates.

Remark 11 There is no appearance of $\chi$ in $\Delta_{\Gamma_{\star}}$ because initial data for $\chi$ used in the following calculations are required only on $\mathcal{N}_{\star}$ where $\chi$ is zero.

Remark 12 In addition to the above norms, we recall that the norm $\Delta_{e_{\star}}$, as defined in Eq. (15) has been used to control the initial value of the components of the frame.

Remark 13 Observe that the above expressions do not include any norm for the components of the connection coefficients away from the initial null hypersurfaces. Instead such norms will be controlled by local bootstrap arguments within the proof.

Remark 14 Throughout the proof besides keeping track of $\Delta_{\Psi_{\star}}$ and $\Delta_{\Psi_{\star}}(\mathcal{S})$, to assist in future generalization, we trace also the dependence of our various constants on $I, \Delta_{e_{\star}}, \Delta_{\Gamma_{\star}}, \Delta_{\Psi_{\star}}$. Note that because of the way that we setup our frame none of the constants so far depend upon $I$.

\subsection{Estimates for the connection coefficients}

In this section we show how to construct estimates on the coefficients of the connection. The strategy is an application of the tools developed in Sect. 5.4 to estimate the solutions of generic transport equations along null hypersurfaces. In this approach, as a bootstrap, control is assumed of the curvature (components of the Weyl tensor) on the double foliation of null hypersurfaces and on the 2-spheres of constant $u$ and $v$ through the norms $\Delta_{\Psi}$ and $\Delta_{\Psi}(\mathcal{S})$.

In a first step we obtain basic control of the $L^{\infty}$-norm of the connection coefficients by assuming finiteness of $\Delta_{\Psi}$ and $\Delta_{\Psi}(\mathcal{S})$ and of third derivatives of the NP coefficient $\tau$ in terms of the $L^{2}$-norm on the 2-spheres $\mathcal{S}_{u, v}$.

Proposition 7 (Control on the supremum norm of the connection coefficients) Assume that we have a solution of the vacuum EFEs in Stewart's gauge in a region $\mathcal{D}_{u, v}^{t}$. with

$$
\sup _{u, v}\|\{\mu, \lambda, \alpha, \beta, \epsilon, \rho, \sigma, \tau, \chi\}\|_{L^{\infty}\left(S_{u, v}\right)} \leq \Delta_{\Gamma},
$$


for some positive $\Delta_{\Gamma}$. Assume also

$$
\sup _{u, v}\left\|\nabla^{2} \tau\right\|_{L^{2}\left(S_{u, v}\right)}<\infty, \quad \sup _{u, v}\left\|\not \nabla^{3} \tau\right\|_{L^{2}\left(S_{u, v}\right)}<\infty, \quad \Delta_{\Psi}(\mathcal{S})<\infty, \quad \Delta_{\Psi}<\infty,
$$

on the same domain. Then there exists

$$
\varepsilon_{\star}=\varepsilon_{\star}\left(I, \Delta_{e_{\star}}, \Delta_{\Gamma_{\star}}, \sup _{u, v}\left\|\not \nabla^{2} \tau\right\|_{L^{2}\left(\mathcal{S}_{u, v}\right)}, \sup _{u, v}\left\|\nabla^{3} \tau\right\|_{L^{2}\left(\mathcal{S}_{u, v}\right)}, \Delta_{\Psi}\right),
$$

such that when $\varepsilon \leq \varepsilon_{\star}$, we have

$$
\begin{aligned}
& \sup _{u, v}\|\{\tau, \chi\}\|_{L^{\infty}\left(\mathcal{S}_{u, v}\right)} \leq C\left(I, \Delta_{e_{\star}}, \Delta_{\Gamma_{\star}}, \Delta_{\Psi}(\mathcal{S})\right), \\
& \sup _{u, v}\|\{\mu, \lambda, \alpha, \beta, \epsilon, \rho, \sigma\}\|_{L^{\infty}\left(\mathcal{S}_{u, v}\right)} \leq 3 \Delta_{\Gamma_{\star}},
\end{aligned}
$$

on $\mathcal{D}_{u, v_{\bullet}}^{t}$.

Remark 15 Observe that in the above proposition, as well as in several of the following ones, the NP spin connection coefficient $\tau$ is singled out as it requires additional hypotheses.

Remark 16 The first assumption here covers Assumption 1, which allows us to employ Lemma 5, Corollary 1, Lemma 6, Proposition 3 and the Sobolev inequalities of Propositions 5, 6 and Corollary 3. It also permits the use of Propositions 2 and 4.

Proof Basic bootstrap assumption We start by making the bootstrap assumption

$$
\sup _{u, v} \|\left(\{\mu, \lambda, \alpha, \beta, \epsilon, \rho, \sigma\} \|_{L^{\infty}\left(\mathcal{S}_{u, v}\right)} \leq 4 \Delta_{\Gamma_{\star}}\right.
$$

Estimate for $\tau$. As first step we prove that

$$
\|\tau\|_{L^{\infty}\left(\mathcal{S}_{u, v}\right)} \leq C\left(I, \Delta_{e_{\star}}, \Delta_{\Gamma_{\star}}, \Delta_{\Psi}(\mathcal{S})\right)
$$

For this, we make use of the $D$-Eq. (30b) for the NP coefficient $\tau$ :

$$
D \tau=(\epsilon-\bar{\epsilon}+\rho) \tau+\sigma \bar{\tau}+\bar{\pi} \rho+\pi \sigma+\Psi_{1} .
$$

Making use of the Sobolev inequality in Proposition 6, we readily obtain from our assumptions that for $\varepsilon$ sufficiently small,

$$
\left\|\Psi_{0}, \Psi_{1}, \Psi_{2}, \Psi_{3}, \Psi_{4}\right\|_{L^{\infty}\left(\mathcal{S}_{u, v}\right)} \leq \Delta_{\Psi}(\mathcal{S})<\infty
$$

Moreover, the inequalities in Proposition 3 show that

$$
\|\tau\|_{L^{\infty}\left(\mathcal{S}_{u, v}\right)} \leq\|\tau\|_{L^{\infty}\left(\mathcal{S}_{u, 0}\right)}+\int_{0}^{v}\|D \tau\|_{L^{\infty}\left(\mathcal{S}_{u, v^{\prime}}\right)} \mathrm{d} v^{\prime}
$$




$$
\begin{aligned}
& \leq\|\tau\|_{L^{\infty}\left(\mathcal{S}_{u, 0}\right)}+\int_{0}^{v}\left\|\bar{\pi} \rho+\pi \sigma+\Psi_{1}\right\|_{L^{\infty}\left(\mathcal{S}_{u, v^{\prime}}\right)} \mathrm{d} v^{\prime} \\
& \quad+\int_{0}^{v}|\epsilon-\bar{\epsilon}+\rho|\|\tau\|_{L^{\infty}\left(\mathcal{S}_{u, v^{\prime}}\right)} \mathrm{d} v^{\prime}+\int_{0}^{v}|\sigma|\|\bar{\tau}\|_{L^{\infty}\left(\mathcal{S}_{u, v^{\prime}}\right)} \mathrm{d} v^{\prime} \\
& \leq \Delta_{\Gamma_{\star}}+\left(32 \Delta_{\Gamma_{\star}}^{2}+\Delta_{\Psi}(\mathcal{S})\right) v_{\bullet}+16 \Delta_{\Gamma_{\star}} \int_{0}^{v}\|\tau\|_{L^{\infty}\left(\mathcal{S}_{u, v^{\prime}}\right)} \mathrm{d} v^{\prime} .
\end{aligned}
$$

Using Grönwall's inequality in the previous expression one then concludes that

$$
\|\tau\|_{L^{\infty}\left(\mathcal{S}_{u, v}\right)} \leq C\left(I, \Delta_{e_{\star}}, \Delta_{\Gamma_{\star}}, \Delta_{\Psi}(\mathcal{S})\right)
$$

Estimate for $\chi$ To obtain the estimate for $\chi$ we proceed in a similar manner. We use the $D$-transport equation Eq. (16) for $\chi$ to obtain

$$
\begin{aligned}
& \|\chi\|_{L^{\infty}\left(\mathcal{S}_{u, v}\right)} \leq\|\chi\|_{L^{\infty}\left(\mathcal{S}_{u, 0}\right)}+\int_{0}^{v}\|D \chi\|_{L^{\infty}\left(\mathcal{S}_{u, v^{\prime}}\right)} \mathrm{d} v^{\prime} \\
& \leq\left(2 \Delta_{\Psi}(\mathcal{S})+c \Delta_{\Gamma_{\star}}+C\right) v_{\bullet}+2 \Delta_{\Gamma_{\star}} \int_{0}^{v}\|\chi\|_{L^{\infty}\left(\mathcal{S}_{u, v^{\prime}}\right)} \mathrm{d} v^{\prime},
\end{aligned}
$$

where $c$ is a positive constant and the constant $C$ is related to the constant appearing in the estimate for $\tau$. From the latter, Grönwall's inequality readily yields

$$
\|\chi\|_{L^{\infty}\left(\mathcal{S}_{u, v}\right)} \leq C\left(I, \Delta_{e_{\star}}, \Delta_{\Gamma_{\star}}, \Delta_{\Psi}(\mathcal{S})\right)
$$

Estimates for $\mu$ and $\lambda$ To obtain estimates of the NP coefficients $\mu$ and $\lambda$ we make use of the $\Delta$-transport Eqs. (30g) and (30o):

$$
\begin{aligned}
& \Delta \mu=-\mu^{2}-\lambda \bar{\lambda}, \\
& \Delta \lambda=-2 \mu \lambda-\Psi_{4} .
\end{aligned}
$$

These are Riccati-type equations and, thus, they can only be naively integrated for a small distance in the $u$ direction -i.e. $u \in[0, \varepsilon]$. Now, making use of the inequalities in Proposition 3 we find that

$$
\|\mu\|_{L^{\infty}\left(\mathcal{S}_{u, v}\right)} \leq\|\mu\|_{L^{\infty}\left(\mathcal{S}_{0, v}\right)}+C\left(\Delta_{e_{\star}}\right) \int_{0}^{\varepsilon}\|\Delta \mu\|_{L^{\infty}\left(\mathcal{S}_{u^{\prime}, v}\right)} \mathrm{d} u^{\prime} .
$$


Accordingly, one concludes that

$$
\begin{aligned}
\|\mu\|_{L^{\infty}\left(\mathcal{S}_{u, v}\right)} & \leq\|\mu\|_{L^{\infty}\left(\mathcal{S}_{0, v}\right)}+C\left(\Delta_{e_{\star}}\right) \int_{0}^{\varepsilon}\left\|\mu^{2}+\lambda \bar{\lambda}\right\|_{L^{\infty}\left(\mathcal{S}_{u^{\prime}, v}\right)} \mathrm{d} u^{\prime} \\
& \leq\|\mu\|_{L^{\infty}\left(\mathcal{S}_{0, v}\right)}+32 C\left(\Delta_{e_{\star}}\right) \int_{0}^{\varepsilon} \Delta_{\Gamma_{0}}^{2} \mathrm{~d} u^{\prime} \\
& \leq \Delta_{\Gamma_{\star}}+32 C\left(\Delta_{e_{\star}}\right) \Delta_{\Gamma_{\star}}^{2} \varepsilon
\end{aligned}
$$

For $\lambda$ one obtains that

$$
\begin{aligned}
\|\lambda\|_{L^{\infty}\left(\mathcal{S}_{u, v}\right)} & \leq \Delta_{\Gamma_{\star}}+32 C\left(\Delta_{e_{\star}}\right) \Delta_{\Gamma_{\star}}^{2} \varepsilon+C\left(\Delta_{e_{\star}}\right) \int_{0}^{u}\left\|\Psi_{4}\right\|_{L^{\infty}\left(\mathcal{S}_{u^{\prime}, v}\right)} d u^{\prime} \\
& \leq \Delta_{\Gamma_{\star}}+C\left(\Delta_{e_{\star}}, \Delta_{\Gamma_{\star}}\right) \varepsilon+C\left(\Delta_{e_{\star}}\right) \int_{0}^{u} \sum_{i=0}^{2}\left\|\nabla^{i} \Psi_{4}\right\|_{L^{2}\left(\mathcal{S}_{u^{\prime}, v}\right)} \mathrm{d} u^{\prime},
\end{aligned}
$$

where in the second inequality we have made use of the Sobolev embedding propertysee corollary 3. Now, using Hölder's inequality, we can transform the estimate of $\Psi_{4}$ from one on sphere $\mathcal{S}_{u, v}$ to one on a null hypersurface. More precisely, one has that

$$
\begin{aligned}
\int_{0}^{u}\left\|\nabla^{i} \Psi_{4}\right\|_{L^{2}\left(\mathcal{S}_{u^{\prime}, v}\right)} \mathrm{d} u^{\prime} & =\int_{0}^{u}\left(\int_{\mathcal{S}_{u^{\prime}, v}}\left|\mathbb{X}^{i} \Psi_{4}\right|^{2}\right)^{1 / 2} \mathrm{~d} u^{\prime} \\
& \leq\left(\int_{0}^{u} \int_{\mathcal{S}_{u^{\prime}, v}}\left|\nabla^{i} \Psi_{4}\right|^{2} \mathrm{~d} u^{\prime}\right)^{1 / 2}\left(\int_{0}^{u} 1 \mathrm{~d} u^{\prime}\right)^{1 / 2} \\
& \leq C \varepsilon^{1 / 2}\left\|\nabla^{i} \Psi_{4}\right\|_{L^{2}\left(\mathcal{N}_{v}(0, u)\right)}
\end{aligned}
$$

Hence, we conclude that

$$
\|\lambda\|_{L^{\infty}\left(\mathcal{S}_{u, v}\right)} \leq \Delta_{\Gamma_{\star}}+C\left(\Delta_{e_{\star}}, \Delta_{\Gamma_{\star}}\right) \varepsilon+C \Delta_{\Psi} \varepsilon^{1 / 2} .
$$

Together, the estimates for $\mu$ and $\lambda$ show that the maximum of these functions will not be too far away from their initial value for $\varepsilon$ sufficiently small.

Estimates for $\alpha, \beta$ and $\epsilon$ Estimates $\alpha, \beta$ and $\epsilon$ can be obtained by a similar method-i.e. integration along the short direction. In this case the relevant $\Delta$-transport equations are given by the structure Eqs. (30k), (30d) and (30a),

$$
\begin{aligned}
& \Delta \alpha=-\mu \alpha-\lambda \beta-\lambda \tau-\Psi_{3}, \\
& \Delta \beta=-\bar{\lambda} \alpha-\mu \beta-\tau \mu, \\
& \Delta \epsilon=-\alpha \bar{\pi}-\beta \pi-\alpha \tau-\beta \bar{\tau}-\pi \tau-\Psi_{2},
\end{aligned}
$$

where it is recalled that in the present gauge one has that $\pi=\alpha+\bar{\beta}$ - see Lemma 1 , Eq. (11c). The details are omitted. 
Estimates for $\rho$ and $\sigma$ In this case the relevant $\Delta$-transport equations are the structure Eqs. (30i) and (30r):

$$
\begin{aligned}
\Delta \rho & =\bar{\delta} \tau-\mu \rho-\lambda \sigma-\alpha \tau+\bar{\beta} \tau-\tau \bar{\tau}-\Psi_{2}, \\
\Delta \sigma & =\delta \tau-\bar{\lambda} \rho-\mu \sigma+\bar{\alpha} \tau-\beta \tau-\tau^{2} .
\end{aligned}
$$

Observe that these equations contain the derivatives $\delta \tau$ and $\bar{\delta} \tau$. To control these terms from our hypotheses, we make use of the Sobolev inequalities in corollary 3 which, together with integration by parts on $\mathcal{S}_{u, v}$ allows us to show that,

$$
\begin{aligned}
\|\not \nabla \tau\|_{L^{\infty}\left(\mathcal{S}_{u, v}\right)} & \leq C\left(\Delta_{e_{\star}}\right) \sum_{i=1}^{3}\left\|\mathbb{D}^{i} \tau\right\|_{L^{2}\left(\mathcal{S}_{u, v}\right)} \\
& \leq C\left(\Delta_{e_{\star}}\right)\left(\|\tau\|_{L^{2}\left(\mathcal{S}_{u, v}\right)}+\left\|\not \nabla^{2} \tau\right\|_{L^{2}\left(\mathcal{S}_{u, v}\right)}+\left\|\not \nabla^{3} \tau\right\|_{L^{2}\left(\mathcal{S}_{u, v}\right)}\right) .
\end{aligned}
$$

It follows then from the Hölder inequality

$$
\|\tau\|_{L^{2}\left(\mathcal{S}_{u, v}\right)} \leq\|\tau\|_{L^{\infty}\left(\mathcal{S}_{u, v}\right)} \operatorname{Area}\left(\mathcal{S}_{u, v}\right)^{1 / 2}
$$

and the boundedness assumptions on $\left\|\nabla^{i} \tau\right\|_{L^{2}\left(\mathcal{S}_{u, v}\right)}$ for $i=2,3$, that

$$
\|\not \nabla \tau\|_{L^{\infty}\left(\mathcal{S}_{u, v}\right)}<\infty
$$

From this observation, an argument similar to the one used for $\mu$ and $\lambda$ yields the required estimates.

Concluding the argument From the estimates for the NP connection coefficients constructed above it follows that one can choose

$$
\varepsilon=\varepsilon\left(I, \Delta_{e_{\star}}, \Delta_{\Gamma_{\star}}, \sup _{u, v}\left\|\nabla^{2} \tau\right\|_{L^{2}\left(\mathcal{S}_{u, v}\right)}, \sup _{u, v}\left\|\nabla^{3} \tau\right\|_{L^{2}\left(\mathcal{S}_{u, v}\right)}, \Delta_{\Psi}(\mathcal{S}), \Delta_{\Psi}\right)
$$

sufficiently small so that

$$
\sup _{u, v}\|\{\mu, \lambda, \alpha, \beta, \epsilon, \rho, \sigma\}\|_{L^{\infty}\left(\mathcal{S}_{u, v}\right)} \leq 3 \Delta_{\Gamma_{\star}} .
$$

Accordingly, we have improved our initial bootstrap assumption. As this is our first such improvement we give an overview of the technique. Recall that to complete a bootstrap argument we need first, to verify that the hypothesis, in our case that $\sup _{u, v}\|\{\mu, \lambda, \alpha, \beta, \epsilon, \rho, \sigma\}\|_{L^{\infty}\left(\mathcal{S}_{u, v}\right)} \leq 4 \Delta_{\Gamma_{\star}}$ holds over the region of interest, is satisfied. We then need to demonstrate, as in the previous argument, that the hypothesis can be improved for $\varepsilon$ sufficiently small. Obviously if the conclusion $\sup _{u, v}\|\{\mu, \lambda, \alpha, \beta, \epsilon, \rho, \sigma\}\|_{L^{\infty}\left(\mathcal{S}_{u, v}\right)} \leq 3 \Delta_{\Gamma_{\star}}$ holds at some point then our hypothesis holds in a neighborhood of that point. Since the interval $[0, \varepsilon]$ is connected and the set on which our desired conclusion holds is open, closed and non-empty, it follows that the desired conclusion holds for $u \in[0, \varepsilon]$. In the argument above we have 
shown that we can improve the hypothesis from a bound $4 \Delta_{\Gamma_{\star}}$ to $3 \Delta_{\Gamma_{\star}}$. Evidently the same arguments could be used to improve from $(N+1) \Delta_{\Gamma_{\star}}$ to $N \Delta_{\Gamma_{\star}}$ for any natural number $N \geq 3$. Given our initial assumption that $\|\{\mu, \lambda, \alpha, \beta, \epsilon, \rho, \sigma\}\|_{L^{\infty}\left(\mathcal{S}_{u, v}\right)} \leq$ $\Delta_{\Gamma}$ we can therefore choose $N$ so that $\Delta_{\Gamma} \leq N \Delta_{\Gamma_{\star}}$ and iterate from $N$ down to 4 to guarantee that our hypothesis is indeed satisfied in some truncated diamond, demonstrating the statement.

The existence proof also requires control over the $L^{4}$-norms of the $\delta$ and $\bar{\delta}$ derivatives of the NP spin connection coefficients. This is provided by the following:

Proposition 8 (Control on the $L^{4}$-norm of the connection coefficients) Make the same assumptions as in Proposition 7, and additionally assume that,

$$
\sup _{u, v}\|\not \nabla\{\mu, \lambda, \alpha, \beta, \epsilon, \rho, \sigma\}\|_{L^{4}\left(\mathcal{S}_{u, v}\right)} \leq \Delta_{\Gamma}
$$

in the truncated diamond $\mathcal{D}_{u, v_{\bullet}}^{t}$. Then there exists,

$$
\varepsilon_{\star}=\varepsilon_{\star}\left(I, \Delta_{e_{\star}}, \Delta_{\Gamma_{\star}}, \sup _{u, v}\left\|\not \nabla^{2} \tau\right\|_{L^{2}\left(\mathcal{S}_{u, v}\right)}, \sup _{u, v}\left\|\not \nabla^{3} \tau\right\|_{L^{2}\left(\mathcal{S}_{u, v}\right)}, \Delta_{\Psi}(\mathcal{S}), \Delta_{\Psi}\right),
$$

such that when $\varepsilon \leq \varepsilon_{\star}$, we have,

$$
\begin{aligned}
& \sup _{u, v}\|\not \nabla\{\tau, \chi\}\|_{L^{4}\left(\mathcal{S}_{u, v}\right)} \leq C\left(I, \Delta_{e_{\star}}, \Delta_{\Gamma_{\star}}, \Delta_{\Psi}(\mathcal{S})\right), \\
& \sup _{u, v}\|\not \nabla\{\mu, \lambda, \alpha, \beta, \epsilon, \rho, \sigma\}\|_{L^{4}\left(\mathcal{S}_{u, v}\right)} \leq 3 \Delta_{\Gamma_{\star}},
\end{aligned}
$$

on $\mathcal{D}_{u, v_{\bullet}}^{t}$.

Proof Basic bootstrap assumption In order to run the argument we make the following bootstrap assumption:

$$
\sup _{u, v}\|\not \nabla\{\mu, \lambda, \alpha, \beta, \epsilon, \rho, \sigma\}\|_{L^{4}\left(\mathcal{S}_{u, v}\right)} \leq 4 \Delta_{\Gamma_{\star}}
$$

Estimates for $\not \nabla \tau$ First we make use of the boundedness of the $L^{2}$-norm of $\tau$ and its angular derivatives up to third order to estimate the $L^{4}$-norm of the first order angular derivatives of $\tau$. For this, we apply $\delta$ to the $D$-transport equation for $\tau$-Eq. (30b). After making use of the commutators of directional covariant derivatives one arrives at the equations

$$
\begin{aligned}
D \delta \tau= & (\rho+\bar{\rho}+2 \epsilon-2 \bar{\epsilon}) \delta \tau+\sigma \bar{\delta} \tau+\sigma \delta \bar{\tau}+\delta(\epsilon-\bar{\epsilon}+\rho) \tau \\
& +\bar{\tau} \delta \sigma+\rho \delta \bar{\pi}+\bar{\pi} \delta \rho+\sigma \delta \pi+\pi \delta \sigma+\delta \Psi_{1}, \\
D \bar{\delta} \tau= & 2 \rho \bar{\delta} \tau+\sigma \bar{\delta} \bar{\tau}+\bar{\sigma} \delta \tau+\tau \bar{\delta}(\epsilon-\bar{\epsilon}+\rho)+\bar{\tau} \bar{\delta} \sigma \\
& +\rho \bar{\delta} \bar{\pi}+\bar{\pi} \delta \sigma \rho+\sigma \bar{\delta} \pi+\pi \bar{\delta} \sigma+\bar{\delta} \Psi_{1} .
\end{aligned}
$$


The above equation contains terms of the form $\Gamma \not \Gamma$-i.e. products of connection coefficients and their derivatives. In the following the $L^{4}$-norm of these products will be split using the Hölder inequality as follows:

$$
\|\Gamma \not \nabla \Gamma\|_{L^{4}\left(\mathcal{S}_{u, v}\right)} \leq\|\Gamma\|_{L^{\infty}\left(\mathcal{S}_{u, v}\right)}\|\not \nabla \Gamma\|_{L^{4}\left(\mathcal{S}_{u, v}\right)}
$$

Observe that from Proposition 7 it follows that terms of the type $\|\Gamma\|_{L^{\infty}\left(\mathcal{S}_{u, v}\right)}$ are bounded.

Now, making use of the Sobolev inequality in Proposition 5, we obtain that

$$
\sum_{j=0}^{1}\left\|\nabla^{j} \Psi_{i}\right\|_{L^{4}\left(\mathcal{S}_{u, v}\right)} \leq \Delta_{\Psi}(\mathcal{S})<\infty, \quad i=0,1,2,3
$$

Combining this with the inequality in the long direction shown in Proposition 2 we find that

$$
\begin{aligned}
& \|\delta \tau\|_{L^{4}\left(\mathcal{S}_{u, v}\right)}+\|\bar{\delta} \tau\|_{L^{4}\left(\mathcal{S}_{u, v}\right)} \leq C\left(I, \Delta_{\Gamma_{\star}}\right) \\
& \quad\left(\|\delta \tau\|_{L^{4}\left(\mathcal{S}_{u, 0}\right)}+\|\bar{\delta} \tau\|_{L^{4}\left(\mathcal{S}_{u, 0}\right)}+\int_{0}^{v}\|D \delta \tau\|_{L^{4}\left(\mathcal{S}_{u, v^{\prime}}\right)}+\|D \bar{\delta} \tau\|_{L^{4}\left(\mathcal{S}_{u, v^{\prime}}\right)} \mathrm{d} v^{\prime}\right) .
\end{aligned}
$$

Substituting the expressions for $D \delta \tau$ and $D \bar{\delta} \tau$ given by Eqs. (21a)-(21b) one concludes that

$$
\begin{aligned}
& \|\delta \tau\|_{L^{4}\left(\mathcal{S}_{u, v}\right)}+\|\bar{\delta} \tau\|_{L^{4}\left(\mathcal{S}_{u, v}\right)} \\
& \quad \leq C_{1}\left(I, \Delta_{\Gamma_{\star}}, \Delta_{\Psi}(\mathcal{S})\right)+C_{2}\left(I, \Delta_{\Gamma_{\star}}\right) \int_{0}^{v}\left(\|\delta \tau\|_{L^{4}\left(\mathcal{S}_{u, v^{\prime}}\right)}+\|\bar{\delta} \tau\|_{L^{4}\left(\mathcal{S}_{u, v^{\prime}}\right)}\right) \mathrm{d} v^{\prime} .
\end{aligned}
$$

Thus, using Grönwall's inequality it follows that

$$
\|\delta \tau\|_{L^{4}\left(\mathcal{S}_{u, v}\right)}+\|\bar{\delta} \tau\|_{L^{4}\left(\mathcal{S}_{u, v}\right)} \leq C\left(I, \Delta_{\Gamma_{\star}}, \Delta_{\Psi}(\mathcal{S})\right)
$$

Consequently, one has

$$
\|\not \nabla \tau\|_{L^{4}\left(\mathcal{S}_{u, v}\right)} \leq C\left(I, \Delta_{\Gamma_{\star}}, \Delta_{\Psi}(\mathcal{S})\right)
$$

as required.

Estimates for $\not \chi$ From Eq. (16) one can readily compute that

$$
D \delta \chi=(\bar{\rho}-2 \bar{\epsilon}) \delta \chi+\sigma \bar{\delta} \chi+\delta\left(\Psi_{2}+\bar{\Psi}_{2}\right)+\Gamma \delta \Gamma-\chi \delta(\epsilon+\bar{\epsilon}),
$$

where $\Gamma$ represents a combination of connection coefficients whose particular form is not essential. A similar equation for $D \bar{\delta} \chi$ can be computed. Using the same strategy used for $\not \chi$ one concludes from the above equations that, 


$$
\|\delta \chi\|_{L^{4}\left(\mathcal{S}_{u, v}\right)}+\|\bar{\delta} \chi\|_{L^{4}\left(\mathcal{S}_{u, v}\right)} \leq C\left(I, \Delta_{\Gamma_{\star}}, \Delta_{\Psi}(\mathcal{S})\right) .
$$

In other words, we find that

$$
\|\mathbb{\chi} \chi\|_{L^{4}\left(\mathcal{S}_{u, v}\right)} \leq C\left(I, \Delta_{\Gamma_{\star}}, \Delta_{\Psi}(\mathcal{S})\right) .
$$

Estimates for the remaining connection coefficients In order to obtain equations for $\delta \mu$ and $\delta \lambda$, we apply the $\Delta$-directional derivative on both sides of Eqs. (30g) and (30o). This gives,

$$
\begin{aligned}
& \Delta \delta \mu=(\tau-\bar{\alpha}-\beta)\left(\mu^{2}+\lambda \bar{\lambda}\right)-3 \mu \delta \mu-\bar{\lambda} \bar{\delta} \mu-\lambda \delta \bar{\lambda}-\bar{\lambda} \delta \lambda, \\
& \Delta \delta \lambda=(\tau-\bar{\alpha}-\beta)\left(2 \mu \lambda+\Psi_{4}\right)-3 \mu \delta \lambda-\bar{\lambda} \delta \bar{\delta}-2 \lambda \delta \mu-\delta \Psi_{4} .
\end{aligned}
$$

A direct computation using Proposition 2 shows that there exists an $\varepsilon_{\star}$ such that

$$
\|\not \nabla\{\mu, \lambda\}\|_{L^{4}\left(\mathcal{S}_{u, v}\right)} \leq 3 \Delta_{\Gamma_{\star}}
$$

if $\varepsilon \leq \varepsilon_{\star}$. The details of this computation can be found in "Appendix E". We can estimate $\delta \alpha, \delta \beta$ and $\delta \epsilon$ by the same method. Since, by our bootstrap assumption $\sup _{u, v}\left\|\nabla^{3} \tau\right\|_{L^{2}\left(\mathcal{S}_{u, v}\right)}<\infty$, it follows from the Sobolev inequalities in Corollary 3 that $\left\|\nabla^{i} \tau\right\|_{L^{4}\left(\mathcal{S}_{u, v}\right)}$ for $i \leq 2$ are finite. Using this information we can estimate $\delta \rho$ and $\delta \sigma$ applying the $\delta$-directional derivative to Eqs. (30i) and (30r).

Concluding the argument From the previous estimates it follows that we can find an $\varepsilon_{\star}$ depending on $I, \Delta_{e_{\star}}, \Delta_{\Gamma_{\star}}, \sup _{u, v}\left\|\not \nabla^{2} \tau\right\|_{L^{2}\left(\mathcal{S}_{u, v}\right)}, \sup _{u, v}\left\|\nabla^{3} \tau\right\|_{L^{2}\left(\mathcal{S}_{u, v}\right)}, \Delta_{\Psi}(\mathcal{S})$, and $\Delta_{\Psi}$, such that

$$
\sup _{u, v}\|\not \nabla\{\mu, \lambda, \alpha, \beta, \epsilon, \rho, \sigma\}\|_{L^{4}\left(\mathcal{S}_{u, v}\right)} \leq 3 \Delta_{\Gamma_{\star}} .
$$

The bootstrap can hence be closed as in Proposition 7.

In a similar vein, the next proposition shows how to obtain control on the $L^{2}$-norms of the NP connection coefficients and their first and second derivatives.

Proposition 9 (Control on the $L^{2}$-norm of the connection coefficients) Assume that we have a solution of the vacuum EFEs in Stewart's gauge in a region $\mathcal{D}_{u, v}^{t}$. with

$$
\begin{aligned}
\sup _{u, v}\|\{\mu, \lambda, \alpha, \beta, \epsilon, \rho, \sigma, \tau, \chi\}\|_{L^{\infty}\left(S_{u, v}\right)} & \leq \Delta_{\Gamma}, \\
\sup _{u, v}\|\not \nabla\{\mu, \lambda, \alpha, \beta, \epsilon, \rho, \sigma\}\|_{L^{4}\left(\mathcal{S}_{u, v}\right)} & \leq \Delta_{\Gamma}, \\
\sup _{u, v}\left\|\nabla^{2}\{\mu, \lambda, \alpha, \beta, \epsilon, \rho, \sigma, \tau\}\right\|_{L^{2}\left(\mathcal{S}_{u, v}\right)} & \leq \Delta_{\Gamma},
\end{aligned}
$$

for some positive $\Delta_{\Gamma}$. Assume also

$$
\sup _{u, v}\left\|\nabla^{3} \tau\right\|_{L^{2}\left(S_{u, v}\right)}<\infty, \quad \Delta_{\Psi}(\mathcal{S})<\infty, \quad \Delta_{\Psi}<\infty,
$$


on the same domain. We have that there exists

$$
\varepsilon_{\star}=\varepsilon_{\star}\left(I, \Delta_{e_{\star}}, \Delta_{\Gamma_{\star}}, \sup _{u, v}\left\|\nabla^{3} \tau\right\|_{L^{2}\left(\mathcal{S}_{u, v}\right)}, \Delta_{\Psi}(\mathcal{S}), \Delta_{\Psi}\right),
$$

such that when $\varepsilon \leq \varepsilon_{\star}$, we have that

$$
\begin{aligned}
& \sup _{u, v}\left\|\nabla^{2}\{\tau, \chi\}\right\|_{L^{2}\left(\mathcal{S}_{u, v}\right)} \leq C\left(I, \Delta_{e_{\star}}, \Delta_{\Gamma_{\star}}, \Delta_{\Psi}(\mathcal{S})\right), \\
& \sup _{u, v}\left\|\nabla^{2}\{\mu, \lambda, \alpha, \beta, \epsilon, \rho, \sigma\}\right\|_{L^{2}\left(\mathcal{S}_{u, v}\right)} \leq 3 \Delta_{\Gamma_{\star}} .
\end{aligned}
$$

Proof Basic bootstrap assumption Examining the above hypotheses we first observe that both Propositions 7 and 8 are applicable. We start then with the following basic bootstrap assumption:

$$
\sup _{u, v}\left\|\nabla^{2}\{\mu, \lambda, \alpha, \beta, \epsilon, \rho, \sigma\}\right\|_{L^{2}\left(\mathcal{S}_{u, v}\right)} \leq 4 \Delta_{\Gamma_{\star}}
$$

Estimates for $\left\|\nabla^{2} \tau\right\|_{L^{2}\left(\mathcal{S}_{u, v}\right)}$ and $\left\|\nabla^{2} \chi\right\|_{L^{2}\left(\mathcal{S}_{u, v}\right)}$ Starting from Eq. (21a), applying the $\delta$-directional derivative and using the commutators one obtains a $D$-transport equation of the form

$$
D \delta^{2} \tau=\Gamma \delta^{2} \tau+\Gamma \delta^{2} \bar{\tau}+\Gamma \bar{\delta} \delta \tau+\Gamma \delta \bar{\delta} \tau+\delta^{2} \Psi_{1}+\Gamma_{1} \delta^{2} \Gamma_{1}+\delta \Gamma_{1} \delta \Gamma_{1},
$$

where $\Gamma$ depends linearly on $\epsilon, \rho, \sigma$, while $\Gamma_{1}$ depends linearly on $\tau, \alpha, \beta, \epsilon, \rho, \sigma$. Similar computations lead to equations for $D \bar{\delta} \tau$ and $D \delta \bar{\delta} \tau$. The term $\delta \Gamma_{1} \delta \Gamma_{1}$ is dealt with using the Hölder inequality to obtain

$$
\left\|\delta \Gamma_{1} \delta \Gamma_{1}\right\|_{L^{2}\left(\mathcal{S}_{u, v}\right)} \leq\left\|\delta \Gamma_{1}\right\|_{L^{4}\left(\mathcal{S}_{u, v}\right)}\left\|\delta \Gamma_{1}\right\|_{L^{4}\left(\mathcal{S}_{u, v}\right)} .
$$

Using Proposition 8, it follows then that the left-hand side of the inequality is finite.

Now, the inequality in the long direction of Proposition 2 and the equation for $D \delta \tau$ show that,

$$
\begin{aligned}
& \left\|\delta^{2} \tau\right\|_{L^{2}\left(\mathcal{S}_{u, v}\right)} \leq C\left(I, \Delta_{\Gamma_{\star}}\right)\left(\left\|\delta^{2} \tau\right\|_{L^{2}\left(\mathcal{S}_{u, 0}\right)}+\int_{0}^{v}\left\|D \delta^{2} \tau\right\|_{L^{2}\left(\mathcal{S}_{u, v^{\prime}}\right)} \mathrm{d} v^{\prime}\right), \\
& \leq C\left(I, \Delta_{e_{\star}}, \Delta_{\Gamma_{\star}}, \Delta_{\Psi}(\mathcal{S})\right)+C\left(I, \Delta_{e_{\star}}, \Delta_{\Gamma_{\star}}\right) \int_{0}^{v}\left\|\not \nabla^{2} \tau\right\|_{L^{2}\left(\mathcal{S}_{u, v^{\prime}}\right)} \mathrm{d} v^{\prime} .
\end{aligned}
$$

Similar estimates can be obtained for $\bar{\delta}^{2} \tau, \delta \bar{\delta} \tau$ and $\bar{\delta} \delta \tau$.

Recalling the result in Corollary 1 that the area of $\mathcal{S}_{u, v}$ is bounded one can estimate the norm $\|\delta \tau\|_{L^{2}\left(\mathcal{S}_{u, v}\right)}$ by observing that

$$
\|\delta \tau\|_{L^{2}\left(\mathcal{S}_{u, v}\right)} \leq C\left(\Delta_{e_{\star}}, \Delta_{\Gamma_{\star}}\right)\|\delta \tau\|_{L^{4}\left(\mathcal{S}_{u, v}\right)} .
$$


Hence, using Proposition 8 it follows that $\|\delta \tau\|_{L^{2}\left(\mathcal{S}_{u, v}\right)}$ is also finite. Now, from inequality (32) we then obtain that

$$
\left\|\not \nabla^{2} \tau\right\|_{L^{2}\left(\mathcal{S}_{u, v}\right)} \leq C\left(I, \Delta_{e_{\star}}, \Delta_{\Gamma_{\star}}, \Delta_{\Psi}(\mathcal{S})\right)+C\left(I, \Delta_{e_{\star}}, \Delta_{\Gamma_{\star}}\right) \int_{0}^{v}\left\|\not \nabla^{2} \tau\right\|_{L^{2}\left(\mathcal{S}_{u, v^{\prime}}\right)} \mathrm{d} v^{\prime} .
$$

so that using Grönwall's inequality one concludes that

$$
\left\|\not \nabla^{2} \tau\right\|_{L^{2}\left(\mathcal{S}_{u, v}\right)} \leq C\left(I, \Delta_{e_{\star}}, \Delta_{\Gamma_{\star}}, \Delta_{\Psi}(\mathcal{S})\right) .
$$

Estimates for $\left\|\nabla^{2} \chi\right\|_{L^{2}\left(\mathcal{S}_{u, v}\right)}$ An analysis analogous to that for $\tau$, readily shows that $\left\|\nabla^{2} \chi\right\|_{L^{2}\left(\mathcal{S}_{u, v}\right)}$ is bounded.

Estimates for the the remaining spin connection coefficients The remaining connection coefficients can be estimated using the same ideas as in Proposition 7-namely, we first compute equations for $\Delta \delta^{2} \Gamma$ and $\Delta \bar{\delta} \delta \Gamma$ using the NP Ricci identities and the commutators for covariant directional derivatives. In a second step we make use of the short direction inequality of Proposition 2. It then follows that one can choose $\varepsilon$ small enough so that,

$$
\sup _{u, v}\left\|\nabla^{2}\{\mu, \lambda, \alpha, \beta, \epsilon, \rho, \sigma\}\right\|_{L^{2}\left(\mathcal{S}_{u, v}\right)} \leq 3 \Delta_{\Gamma_{\star}},
$$

for,

$$
\varepsilon \leq \varepsilon_{\star}\left(I, \Delta_{e_{\star}}, \Delta_{\Gamma_{\star}}, \sup _{u, v}\left\|\not \nabla^{3} \tau\right\|_{L^{2}\left(\mathcal{S}_{u, v}\right)}, \Delta_{\Psi}(\mathcal{S}), \Delta_{\Psi}\right) .
$$

Details of the generic calculations involved in these last steps are discussed in "Appendix C".

\subsection{A first estimate for the curvature}

Having obtained estimates for the NP spin connection coefficients, we are now in the position to obtain a first estimate for the curvature. The proposition of this section provides for bounds the components of the Weyl tensor of the spheres $\mathcal{S}_{u, v}$ assuming, as a bootstrap, their boundedness on the null hypersurfaces and boundedness on $\tau$ and its derivatives.

Proposition 10 (Basic control of the curvature) Assume that we are given a solution to the vacuum EFEs in Stewart's gauge satisfying the assumptions of Proposition 9.

Then there exists

$$
\varepsilon_{\star}=\varepsilon_{\star}\left(\Delta_{e_{\star}}, \Delta_{\Gamma_{\star}}, \Delta_{\Psi_{\star}}, \sup _{u, v}\left\|\nabla^{3} \tau\right\|_{L^{2}\left(\mathcal{S}_{u, v}\right)}, \Delta_{\Psi}\right)
$$


such that for $\varepsilon \leq \varepsilon_{\star}$, one has

$$
\Delta_{\Psi}(\mathcal{S}) \leq C\left(\Delta_{\Psi_{\star}}\right)
$$

on $\mathcal{D}_{u, v_{\bullet}}^{t}$.

Proof Boostrap assumption In this proof we start with the following bootstrap assumption:

$$
\sup _{u, v}\left\|\nabla^{i}\left\{\Psi_{0}, \Psi_{1}, \Psi_{2}, \Psi_{3}\right\}\right\|_{L^{2}\left(\mathcal{S}_{u, v}\right)} \leq 4 \Delta_{\Psi_{\star}}, \quad i=0, \ldots, 2
$$

which we then aim to improve.

$L^{2}$-norm of the components $\left\{\Psi_{0}, \Psi_{1}, \Psi_{2}, \Psi_{3}\right\}$. Estimates for the $L^{2}$-norms of the components $\left\{\Psi_{0}, \Psi_{1}, \Psi_{2}, \Psi_{3}\right\}$ can be obtained from the $\Delta$-Bianchi identity Eqs. (30b), (30h), (30f) and (30d) which are then integrated along the short direction. As an example of the procedure we consider here the coefficient $\Psi_{2}$. From Proposition 2 it follows that

$$
\begin{aligned}
\left\|\Psi_{2}\right\|_{L^{2}\left(\mathcal{S}_{u, v}\right) \leq} & 2\left(\left\|\Psi_{2}\right\|_{L^{2}\left(\mathcal{S}_{0, v}\right)}+C\left(\Delta_{e_{\star}}, \Delta_{\Gamma_{\star}}\right) \int_{0}^{u}\left\|\Delta \Psi_{2}\right\|_{L^{2}\left(\mathcal{S}_{u^{\prime}, v}\right)} \mathrm{d} u^{\prime}\right) \\
\leq & 2\left(\Delta_{\Psi_{\star}}+C\left(\Delta_{e_{\star}}, \Delta_{\Gamma_{\star}}\right) \int_{0}^{u}\left\|\not \nabla \Psi_{3}\right\|_{L^{2}\left(\mathcal{S}_{u^{\prime}, v}\right)}+\left\|3 \mu \Psi_{2}\right\|_{L^{2}\left(\mathcal{S}_{u^{\prime}, v}\right)}\right. \\
& \left.+\left\|2(\beta-\tau) \Psi_{3}\right\|_{L^{2}\left(\mathcal{S}_{u^{\prime}, v}\right)}+\left\|\sigma \Psi_{4}\right\|_{L^{2}\left(\mathcal{S}_{u^{\prime}, v}\right)} \mathrm{d} u^{\prime}\right) \\
\leq & 2\left(\Delta_{\Psi_{\star}}+C\left(\Delta_{e_{\star}}, \Delta_{\Gamma_{\star}}, \Delta_{\Psi_{\star}}\right) \varepsilon+C\left(\Delta_{e_{\star}}, \Delta_{\Gamma_{\star}}\right) \Delta_{\Psi} \varepsilon^{1 / 2}\right. \\
& \left.+C\left(\Delta_{e_{\star}}, \Delta_{\Gamma_{\star}}\right)\left\|\Psi_{4}\right\|_{L^{2}\left(\mathcal{N}_{v}(0, u)\right)} \varepsilon^{1 / 2}\right) \\
\leq & 2 \Delta_{\Psi_{\star}}+C\left(\Delta_{e_{\star}}, \Delta_{\Gamma_{\star}}, \Delta_{\Psi_{\star}}\right) \varepsilon+C\left(\Delta_{e_{\star}}, \Delta_{\Gamma_{\star}}, \Delta_{\Psi_{\star}}\right) \Delta_{\Psi} \varepsilon^{1 / 2}
\end{aligned}
$$

In passing from the second to the third inequality we have used that the term

$$
\int_{0}^{u}\left\|\not \nabla \Psi_{3}\right\|_{L^{2}\left(\mathcal{S}_{u^{\prime}, v}\right)} \mathrm{du}
$$

is, in fact, an statement on the light cone and, hence, it is controlled by the definition of $\Delta_{\Psi}$. Moreover, we have also used Hölder's inequality in the form

$$
\int_{0}^{u}\left\|\Psi_{4}\right\|_{L^{2}\left(\mathcal{S}_{u^{\prime}, v}\right)} \mathrm{d} u^{\prime} \leq C \varepsilon^{1 / 2}\left\|\Psi_{4}\right\|_{L^{2}\left(\mathcal{N}_{v^{\prime}}(0, u)\right)} .
$$

The analysis for the coefficients $\Psi_{0}, \Psi_{1}, \Psi_{3}$ is similar. Consequently, we can find $\varepsilon_{\star}$ depending on the initial data, $\Delta_{\Psi}$ and $I$ such that for $\varepsilon \leq \varepsilon_{\star}$, we have

$$
\sup _{u, v}\left\|\left\{\Psi_{0}, \Psi_{1}, \Psi_{2}, \Psi_{3}\right\}\right\|_{L^{2}\left(\mathcal{S}_{u, v}\right)} \leq 3 \Delta_{\Psi_{\star}}
$$


Estimates for $\left\|\not \backslash\left\{\Psi_{0}, \Psi_{1}, \Psi_{2}, \Psi_{3}\right\}\right\|_{L^{2}\left(\mathcal{S}_{u, v}\right)}$ Again, we focus our discussion on the analysis of the coefficient $\Psi_{2}$. From Proposition 2 we find that

$$
\begin{aligned}
\left\|\not \nabla \Psi_{2}\right\|_{L^{2}\left(\mathcal{S}_{u, v}\right)} & \leq 2\left(\left\|\not \nabla \Psi_{2}\right\|_{L^{2}\left(\mathcal{S}_{0, v}\right)}+C\left(\Delta_{e_{\star}}, \Delta_{\Gamma_{\star}}\right) \int_{0}^{u}\left(\int_{\mathcal{S}_{u^{\prime}, v}} \Delta\left\langle\not \nabla \Psi_{2}, \not \nabla \Psi_{2}\right\rangle_{\sigma}\right)^{1 / 2} \mathrm{~d} u^{\prime}\right) \\
& \leq 2 \Delta \Psi_{\star}+C\left(\Delta_{e_{\star}}, \Delta_{\Gamma_{\star}}\right) \int_{0}^{u}\left(\int_{\mathcal{S}_{u^{\prime}, v}}\left|\not \nabla \Psi_{2}\right|\left(\left|\Delta \delta \Psi_{2}\right|+\left|\Delta \bar{\delta} \Psi_{2}\right|\right)\right)^{1 / 2} \mathrm{~d} u^{\prime} .
\end{aligned}
$$

Now, using the expression for $\Delta \delta \Psi_{2}$ and $\Delta \bar{\delta} \Psi_{2}$ obtained from using the commutators on the $\Delta$-Bianchi equation for $\Psi_{2}$, and schematically denoting arbitrary connection coefficients by $\Gamma$, one obtains that

$$
\begin{aligned}
& \int_{0}^{u}\left(\int_{\mathcal{S}_{u^{\prime}, v}}\left|\not \nabla \Psi_{2}\right|\left(\left|\Delta \delta \Psi_{2}\right|+\left|\Delta \bar{\delta} \Psi_{2}\right|\right)\right)^{1 / 2} \mathrm{~d} u^{\prime} \\
& \leq \int_{0}^{u}\left(\left.\int_{\mathcal{S}_{u^{\prime}, v}}|\not| \Psi_{2}|| \Gamma\right|^{2}\left|\Psi_{2,3}\right|\right)^{1 / 2} \mathrm{~d} u^{\prime}+\int_{0}^{u}\left(\int_{\mathcal{S}_{u^{\prime}, v}}\left|\nabla \Psi_{2}\right||\Gamma|^{2}\left|\Psi_{4}\right|\right)^{1 / 2} \mathrm{~d} u^{\prime} \\
& \quad+\int_{0}^{u}\left(\int_{\mathcal{S}_{u^{\prime}, v}}\left|\not \nabla \Psi_{2}\right||\Gamma||\not| \Psi_{2,3} \mid\right)^{1 / 2} \mathrm{~d} u^{\prime}+\int_{0}^{u}\left(\int_{\mathcal{S}_{u^{\prime}, v}}\left|\not \nabla \Psi_{2}\right||\Gamma||\not| \Psi_{4} \mid\right)^{1 / 2} \mathrm{~d} u^{\prime} \\
& \quad+\int_{0}^{u}\left(\int_{\mathcal{S}_{u^{\prime}, v}}\left|\not \nabla \Psi_{2}\right||\not| \Gamma|| \Psi_{2,3} \mid\right)^{1 / 2} \mathrm{~d} u^{\prime}+\int_{0}^{u}\left(\int_{\mathcal{S}_{u^{\prime}, v}}|\not| \Psi_{2}|| \not \Gamma|| \Psi_{4} \mid\right)^{1 / 2} \mathrm{~d} u^{\prime} \\
& \quad+\int_{0}^{u}\left(\int_{\mathcal{S}_{u^{\prime}, v}}\left|\not \nabla \Psi_{2}\right|\left|\nabla^{2} \Psi_{3}\right|\right)^{1 / 2} \mathrm{~d} u^{\prime} .
\end{aligned}
$$

In the first and third terms of the right-hand side or the above inequality we can separate the $L^{\infty}$-norm of the connection coefficients. Thus, using the bootstrap assumption with Proposition 7, we find that

$$
\begin{aligned}
& \int_{0}^{u}\left(\int_{\mathcal{S}_{u^{\prime}, v}}\left|\not \nabla \Psi_{2}\right||\Gamma|^{2-i}\left|\nabla^{i} \Psi_{2,3}\right|\right)^{1 / 2} \mathrm{~d} u^{\prime} \\
& \quad \leq C\left(I, \Delta_{e_{\star}}, \Delta_{\Gamma_{\star}}, \Delta_{\Psi_{\star}}\right) \int_{0}^{u}\left\|\left.\not \nabla \Psi_{2}\right|_{L^{2}\left(\mathcal{S}_{u^{\prime}, v}\right)} ^{1 / 2}\right\| \nabla^{i} \Psi_{2,3} \|_{L^{2}\left(\mathcal{S}_{u^{\prime}, v}\right)}^{1 / 2} \mathrm{~d} u^{\prime},
\end{aligned}
$$

for $i=0,1$. Accordingly, using the bootstrap assumption once again, we conclude that,

$$
\int_{0}^{u}\left(\int_{\mathcal{S}_{u^{\prime}, v}}\left|\nabla^{i} \Psi_{2}\right||\Gamma|^{2-i}\left|\not \nabla \Psi_{2,3}\right|\right)^{1 / 2} \mathrm{~d} u^{\prime} \leq C\left(I, \Delta_{e_{\star}}, \Delta_{\Gamma_{\star}}, \Delta_{\Psi_{\star}}\right) \varepsilon,
$$

for $i=0,1$. The second and fourth term in the right-hand side of inequality (22) can be handled in an analogous manner. Since we do not have control on the the $L^{2}\left(\mathcal{S}_{u, v}\right)$ 
norm of $\Psi_{4}$, we transform the $L^{2}\left(\mathcal{S}_{u, v}\right)$ norm to a norm over the light cone. More precisely, one has that using Hölder's inequality

$$
\begin{aligned}
\int_{0}^{u}\left(\int_{\mathcal{S}_{u^{\prime}, v}}\left|\nabla \Psi_{2}\right||\Gamma|^{2-i}\left|\nabla^{i} \Psi_{4}\right|\right)^{1 / 2} \mathrm{~d} u^{\prime} & \leq \int_{0}^{u}\left\|\not \nabla \Psi_{2}\right\|_{L^{2}\left(\mathcal{S}_{u^{\prime}, v}\right)}^{1 / 2}\left\|\nabla^{i} \Psi_{4}\right\|_{L^{2}\left(\mathcal{S}_{u^{\prime}, v}\right)}^{1 / 2} \mathrm{~d} u^{\prime} \\
& \leq C\left(\Delta \Psi_{\star}\right)\left\|\nabla^{i} \Psi_{4}\right\|_{L^{2}\left(\mathcal{N}_{v}(0, u)\right)}^{1 / 2} \varepsilon^{3 / 4}, i=0,1
\end{aligned}
$$

Hence, we conclude that

$$
\begin{aligned}
& \int_{0}^{u}\left(\int_{\mathcal{S}_{u^{\prime}, v}}\left|\not \nabla \Psi_{2}\right||\Gamma|^{2}\left|\Psi_{4}\right|\right)^{1 / 2} \mathrm{~d} u^{\prime}, \quad \int_{0}^{u}\left(\int_{\mathcal{S}_{u^{\prime}, v}}\left|\not \nabla \Psi_{2}\right||\Gamma|\left|\not \Psi_{4}\right|\right)^{1 / 2} \mathrm{~d} u^{\prime} \\
& \quad \leq C\left(\Delta_{\Psi_{\star}}, \Delta_{\Psi}\right) \varepsilon^{3 / 4}
\end{aligned}
$$

Now, for the fifth term in inequality (22) one has that

$$
\begin{aligned}
& \int_{0}^{u}\left(\int_{\mathcal{S}_{u^{\prime}, v}}\left|\not \nabla \Psi_{2}\right||\not \nabla \Gamma|\left|\Psi_{2,3}\right|\right)^{1 / 2} \mathrm{~d} u^{\prime} \\
& \quad \leq \int_{0}^{u}\left(\left\|\Psi_{2,3}\right\|_{L^{\infty}\left(\mathcal{S}_{u, v}\right)}|| \not \nabla \Psi_{2}\left\|_{L^{2}\left(\mathcal{S}_{u, v}\right)}\right\| \not \nabla \Gamma \|_{L^{2}\left(\mathcal{S}_{u, v}\right)}\right)^{1 / 2} \mathrm{~d} u^{\prime}
\end{aligned}
$$

where the first term in the integral in the right-hand side can be controlled by the bootstrap assumption and Sobolev embedding (Corollary 3). The third term can be controlled by the $L^{4}\left(\mathcal{S}_{u, v}\right)$ norm as given by Proposition 8 , again in combination with the bootstrap assumption. One then concludes that,

$$
\begin{aligned}
\int_{0}^{u}\left(\int_{\mathcal{S}_{u^{\prime}, v}}\left|\not \nabla \Psi_{2}\right||\nabla \Gamma|\left|\Psi_{2,3}\right|\right)^{1 / 2} \mathrm{~d} u^{\prime} & \leq C\left(I, \Delta_{e_{\star}}, \Delta_{\Gamma_{\star}}, \Delta_{\Psi_{\star}}\right) \sum_{i=0}^{2} \int_{0}^{u}\left\|\nabla^{i} \Psi_{2,3}\right\|_{L^{2}\left(\mathcal{S}_{u^{\prime}, v}\right)}^{1 / 2} \mathrm{~d} u^{\prime} \\
& \leq C\left(I, \Delta_{e_{\star}}, \Delta_{\Gamma_{\star}}, \Delta_{\Psi_{\star}}, \Delta_{\Psi}\right) \varepsilon^{3 / 4}
\end{aligned}
$$

The sixth term in inequality (22) can also be dealt with by transforming the norms of the coefficients of the Weyl tensor on $\mathcal{S}_{u, v}$ to norms on the light cone. More precisely, one has that

$$
\begin{gathered}
\int_{0}^{u}\left(\int_{\mathcal{S}_{u^{\prime}, v}}\left|\not \nabla \Psi_{2}\right||\not \nabla \Gamma|\left|\Psi_{4}\right|\right)^{1 / 2} \mathrm{~d} u^{\prime} \leq \int_{0}^{u}\left(\left\|\Psi_{4}\right\|_{L^{\infty}\left(\mathcal{S}_{u, v}\right)}\left\|\not \nabla \Psi_{2}\right\|_{L^{2}\left(\mathcal{S}_{u, v}\right)}\|\not \nabla \Gamma\|_{L^{2}\left(\mathcal{S}_{u, v}\right)}\right)^{1 / 2} \mathrm{~d} u^{\prime} \\
\quad \leq C\left(I, \Delta_{e_{\star}}, \Delta_{\Gamma_{\star}}, \Delta_{\Psi_{\star}}\right)\left(\int_{0}^{u} \sum_{i=0}^{2}\left\|\nabla^{i} \Psi_{4}\right\|_{L^{2}\left(\mathcal{S}_{u, v}\right)} \mathrm{d} u^{\prime}\right)^{1 / 2} \\
\quad \leq C\left(I, \Delta_{e_{\star}}, \Delta_{\Gamma_{\star}}, \Delta_{\Psi_{\star}}\right)\left(\sum_{i=0}^{2}\left\|\mathbb{X}^{i} \Psi_{4}\right\|_{L^{2}\left(\mathcal{N}_{v}(0, u)\right)}\right) \leq C\left(I, \Delta_{e_{\star}}, \Delta_{\Gamma_{\star}}, \Delta_{\Psi_{\star}}, \Delta_{\Psi}\right) \varepsilon^{3 / 4} .
\end{gathered}
$$


Finally, the last integral in the right-hand side of inequality (22) can be separated into two $L^{2}$-norms. The estimate of $\chi^{2} \Psi_{3}$ can, in turn, be transformed to an estimate on the light cone and, hence, it can be controlled by the definition of $\Delta_{\Psi}$.

Collecting all the estimates for the various terms in inequality (22) we conclude that,

$$
\left\|\not \nabla \Psi_{2}\right\|_{L^{2}\left(\mathcal{S}_{u, v}\right)} \leq 2 \Delta_{\Psi_{\star}}+C\left(I, \Delta_{e_{\star}}, \Delta_{\Gamma_{\star}}, \Delta_{\Psi_{\star}}\right) \varepsilon+C\left(I, \Delta_{e_{\star}}, \Delta_{\Gamma_{\star}}, \Delta_{\Psi_{\star}}, \Delta_{\Psi}\right) \Delta_{\Psi} \varepsilon^{3 / 4} .
$$

The latter inequality implies that we can improve the bootstrap assumption by choosing $\varepsilon$ small enough. A similar strategy allows us to estimate $\left.\not \nabla \Psi_{0}, \Psi_{1}, \Psi_{3}\right\}$. Therefore we have that

$$
\sup _{u, v}\left\|\not \nabla\left\{\Psi_{0}, \Psi_{1}, \Psi_{2}, \Psi_{3}\right\}\right\|_{L^{2}\left(S_{u, v}\right)} \leq 3 \Delta_{\Psi_{\star}}
$$

Estimates for $\left\|\nabla^{2}\left\{\Psi_{0}, \Psi_{1}, \Psi_{2}, \Psi_{3}\right\}\right\|_{L^{2}\left(\mathcal{S}_{u, v}\right)}$ As before, we focus the discussion on $\left\|\not \nabla^{2} \Psi_{2}\right\|_{L^{2}\left(\mathcal{S}_{u, v}\right)}$. The estimate along the short direction in Proposition 2 shows that

$$
\begin{aligned}
\left\|\nabla^{2} \Psi_{2}\right\|_{L^{2}\left(\mathcal{S}_{u, v}\right) \leq} & 2\left(\left\|\nabla^{2} \Psi_{2}\right\|_{L^{2}\left(\mathcal{S}_{0, v}\right)}+C\left(\Delta_{e_{\star}}, \Delta_{\Gamma_{\star}}\right)\right. \\
& \left.\int_{0}^{u}\left(\int_{\mathcal{S}_{u^{\prime}, v}} \Delta\left\langle\nabla^{2} \Psi_{2}, \nabla^{2} \Psi_{2}\right\rangle_{\sigma}\right)^{1 / 2} \mathrm{~d} u^{\prime}\right) \\
\leq & 2 \Delta_{\Psi_{\star}}+C\left(\Delta_{e_{\star}}, \Delta_{\Gamma_{\star}}\right) \int_{0}^{u}\left(\int_{\mathcal{S}_{u^{\prime}, v}}\left|\nabla^{2} \Psi_{2}\right|(|\Delta T|)\right)^{1 / 2} \mathrm{~d} u^{\prime},
\end{aligned}
$$

where $T$ denotes an expression involving products of connection coefficients, their derivatives and components of the Weyl tensor and their derivatives. In particular, one has that

$$
\begin{aligned}
\int_{\mathcal{S}_{u^{\prime}, v}}\left|\not \nabla^{2} \Psi_{2}\right|(|\Delta T|) \leq & \int_{S_{u^{\prime}, v}}\left|\not^{2} \Psi_{2}\right| \mid \Psi \not \nabla^{2} \Gamma+\Gamma \not \nabla^{2} \Psi+\not \nabla \Psi \not \nabla \Gamma \\
& +\Gamma^{2} \not \nabla \Psi+\Gamma \Psi \not \nabla \Psi+\Gamma^{3} \Psi+\Psi_{3} \not \nabla \Psi_{2}+\not \nabla^{3} \Psi_{3} \mid .
\end{aligned}
$$

We can then proceed with a strategy similar to that used in the analysis of the estimates for the first order derivatives of the components of the Weyl tensor. In particular, we use Hölder's inequality to split products and then apply the Sobolev embedding theorem as necessary. The estimates on the sphere for the terms $\not^{i} \Psi_{4}$ and $\not^{3} \Psi_{3}$ are transformed into estimates on the light cone. Hence the integral on the right-handside of inequality (23) can be made as small as necessary by choosing a suitable $\varepsilon$. Ultimately, we conclude that

$$
\sup _{u, v}\left\|\nabla^{2}\left\{\Psi_{0}, \Psi_{1}, \Psi_{2}, \Psi_{3}\right\}\right\|_{L^{2}\left(\mathcal{S}_{u, v}\right)} \leq 3 \Delta_{\Psi_{\star}}
$$


Concluding the argument Collecting all the estimates in the previous steps one obtains the statement

$$
\sup _{u, v}\left\|\nabla^{i}\left\{\Psi_{0}, \Psi_{1}, \Psi_{2}, \Psi_{3}\right\}\right\|_{L^{2}\left(\mathcal{S}_{u, v}\right)} \leq 3 \Delta_{\Psi_{\star}}, \quad i=0, \ldots, 2
$$

which improves the starting bootstrap assumption.

Applying the standard embedding of $L^{p}$ into $L^{q}$ for $p \leq q$, we can summarise the results of Propositions 7, 8, 9 and 10 in the following proposition:

Proposition 11 (Summary of the basic estimates for the NP quantities) Suppose we are given a solution to the vacuum EFE's in Stewart's gauge emanating from data for the CIVP as prepared in Lemma 2, satisfying

$$
\begin{aligned}
& \sup _{u, v}\|\{\mu, \lambda, \alpha, \beta, \epsilon, \rho, \sigma, \tau, \chi\}\|_{L^{\infty}\left(\mathcal{S}_{u, v}\right)}<\infty, \quad \sup _{u, v}\|\not \nabla\{\mu, \lambda, \alpha, \beta, \epsilon, \rho, \sigma\}\|_{L^{4}\left(\mathcal{S}_{u, v}\right)}<\infty, \\
& \sup _{u, v}\left\|\nabla^{2}\{\mu, \lambda, \alpha, \beta, \epsilon, \rho, \sigma, \tau\}\right\|_{L^{2}\left(\mathcal{S}_{u, v}\right)}<\infty, \quad \sup _{u, v}\left\|\not^{3} \tau\right\|_{L^{2}\left(\mathcal{S}_{u, v}\right)}<\infty, \\
& \Delta_{\Psi}(\mathcal{S})<\infty, \quad \Delta_{\Psi}<\infty,
\end{aligned}
$$

on some truncated causal diamond $\mathcal{D}_{u, v}^{t}$. Then there exists,

$$
\varepsilon_{\star}=\varepsilon_{\star}\left(I, \Delta_{e_{\star}}, \Delta_{\Gamma_{\star}}, \Delta_{\Psi_{\star}}, \sup _{u, v}\left\|\not \nabla^{3} \tau\right\|_{L^{2}\left(\mathcal{S}_{u, v}\right)}, \Delta_{\Psi}\right),
$$

such that for $\varepsilon \leq \varepsilon_{\star}$, we have

$$
\begin{array}{ll}
\|\Gamma\|_{L^{\infty}\left(\mathcal{S}_{u, v}\right)} \leq C\left(I, \Delta_{e_{\star}}, \Delta_{\Gamma_{\star}}, \Delta_{\Psi_{\star}}\right), & \sum_{i=0}^{1}\left\|\nabla^{i} \Gamma\right\|_{L^{4}\left(\mathcal{S}_{u, v}\right)} \leq C\left(I, \Delta_{e_{\star}}, \Delta_{\Gamma_{\star}}, \Delta_{\Psi_{\star}}\right), \\
\sum_{i=0}^{2}\left\|\nabla^{i} \Gamma\right\|_{L^{2}\left(\mathcal{S}_{u, v}\right) \leq C\left(I, \Delta_{e_{\star}}, \Delta_{\Gamma_{\star}}, \Delta_{\Psi_{\star}}\right),} & \Delta_{\Psi}(\mathcal{S}) \leq C\left(\Delta_{\Psi_{\star}}\right),
\end{array}
$$

on $\mathcal{D}_{u, v_{\bullet}}^{t}$, with $\Gamma$ standing for an arbitrary connection coefficient.

\subsection{Estimates on the third derivatives of connection coefficients}

We are now in the position to obtain estimates for the NP spin connection coefficients which only require assumptions on the curvature on the light cone. More precisely, one has the following:

Proposition 12 (Further control on the $L^{2}$-norm of the connection coefficients) Assume, as in the previous proposition, that we are given a solution to the vacuum EFE's in Stewart's gauge emanating from data for the CIVP as prepared in Lemma 2. Suppose that, 


$$
\begin{array}{ll}
\sup _{u, v}\|\{\mu, \lambda, \alpha, \beta, \epsilon, \rho, \sigma, \tau, \chi\}\|_{L^{\infty}\left(\mathcal{S}_{u, v}\right)}<\infty, & \sup _{u, v}\|\not \mathcal{X}\{\mu, \lambda, \alpha, \beta, \epsilon, \rho, \sigma\}\|_{L^{4}\left(\mathcal{S}_{u, v}\right)}<\infty, \\
\sup _{u, v}\left\|\nabla^{2}\{\mu, \lambda, \alpha, \beta, \epsilon, \rho, \sigma, \tau\}\right\|_{L^{2}\left(\mathcal{S}_{u, v}\right)}<\infty, & \Delta_{\Psi}(\mathcal{S})<\infty, \quad \Delta_{\Psi}<\infty,
\end{array}
$$

and furthermore that,

$$
\sup _{u, v}\left\|\nabla^{3}\{\mu, \lambda, \alpha, \beta, \epsilon, \tau\}\right\|_{L^{2}\left(\mathcal{S}_{u, v}\right)}<\infty,
$$

on $\mathcal{D}_{u, v_{\bullet}}^{t}$. Then there exists $\varepsilon_{\star}=\varepsilon_{\star}\left(I, \Delta_{e_{\star}}, \Delta_{\Gamma_{\star}}, \Delta_{\Psi_{\star}}, \Delta_{\Psi}\right)$ such that for $\varepsilon \leq \varepsilon_{\star}$, we have

$$
\begin{aligned}
& \sup _{u, v}\left\|\nabla^{3}\{\mu, \lambda, \alpha, \beta, \epsilon\}\right\|_{L^{2}\left(\mathcal{S}_{u, v}\right)} \leq 3 \Delta_{\Gamma_{\star}}, \\
& \sup _{u, v}\left\|\nabla^{3}\{\rho, \sigma\}\right\|_{L^{2}\left(\mathcal{S}_{u, v}\right)} \leq C\left(I, \Delta_{e_{\star}}, \Delta_{\Gamma_{\star}}, \Delta_{\Psi_{\star}}\right), \\
& \sup _{u, v}\left\|\nabla^{3}\{\tau, \chi\}\right\|_{L^{2}\left(\mathcal{S}_{u, v}\right)} \leq C\left(I, \Delta_{e_{\star}}, \Delta_{\Gamma_{\star}}, \Delta_{\Psi_{\star}}, \Delta_{\Psi}\right) .
\end{aligned}
$$

Proof Bootstrap assumption In order to start the proof we place bootstrap assumptions on $\mu, \lambda, \alpha, \beta$ and $\epsilon$, and name the bound on $\tau$ as follows,

$$
\sup _{u, v}\left\|\nabla^{3}\{\mu, \lambda, \alpha, \beta, \epsilon\}\right\|_{L^{2}\left(\mathcal{S}_{u, v}\right)} \leq 4 \Delta_{\Gamma_{\star}}, \quad \sup _{u, v}\left\|\nabla^{3} \tau\right\|_{L^{2}\left(\mathcal{S}_{u, v}\right)} \leq \Delta_{\tau} .
$$

Estimates for $\rho$ and $\sigma$ We first estimate the spin connection coefficients $\rho$ and $\sigma$ using the long direction transport Eqs. (30m) and (30f) as this allows to avoid higher derivatives on the sphere that arise in the short direction equations. Using the expression for $\left\|\not{ }^{3} f\right\|_{L^{2}\left(S_{u, v}\right)}$ for an arbitrary scalar $f$ given in "Appendix C", we will discuss four typical terms. The first is $\delta^{3} \rho$. Making use of the commutators of directional covariant derivatives, we can compute the long direction derivative of any third derivatives of $\rho$ on the sphere-for example, one has that,

$$
\begin{aligned}
D \delta^{3} \rho= & \Gamma^{5}+\Gamma^{3} \delta \Gamma+\Gamma(\delta \Gamma)^{2}+\Gamma^{2} \delta^{2} \Gamma+\delta \Gamma \delta^{2} \Gamma+\rho \delta^{3}(\epsilon+\bar{\epsilon}) \\
& +(4 \epsilon-2 \bar{\epsilon}+5 \rho) \delta^{3} \rho+\sigma \delta^{3} \bar{\sigma}+\bar{\sigma} \delta^{3} \sigma+\sigma \delta^{2} \bar{\delta} \rho,
\end{aligned}
$$

where here $\Gamma$ represents linear combinations of the coefficients $\epsilon, \rho$ and $\sigma$, whose precise form is not crucial for the discussion. The $L^{2}$-norm of the term $\delta \Gamma \delta^{2} \Gamma$ can be split as

$$
\left\|\delta \Gamma \delta^{2} \Gamma\right\|_{L^{2}\left(\mathcal{S}_{u, v}\right)} \leq\|\not \nabla \Gamma\|_{L^{4}\left(\mathcal{S}_{u, v}\right)}\left\|\not \nabla^{2} \Gamma\right\|_{L^{4}\left(\mathcal{S}_{u, v}\right)} .
$$

The first term on the right-hand side of the inequality can be controlled using the results of Proposition 8. The second term can be controlled using the Sobolev inequality,

$$
\left\|\not \nabla^{2} \Gamma\right\|_{L^{4}\left(\mathcal{S}_{u, v}\right)} \leq C\left(\Delta_{e_{\star}}\right)\left(\left\|\not \nabla^{2} \Gamma\right\|_{L^{2}\left(\mathcal{S}_{u, v}\right)}+\left\|\not \nabla^{3} \Gamma\right\|_{L^{2}\left(\mathcal{S}_{u, v}\right)}\right) .
$$


Proceeding in a similar way with the other terms in the equation for $D \delta^{3} \rho$ and the using the long direction inequality in Proposition 2 leads to

$$
\begin{aligned}
\left\|\delta^{3} \rho\right\|_{L^{2}\left(\mathcal{S}_{u, v}\right) \leq} & C\left(I, \Delta_{e_{\star}}, \Delta_{\Gamma_{\star}}, \Delta_{\Psi_{\star}}\right)+C\left(I, \Delta_{e_{\star}}, \Delta_{\Gamma_{\star}}, \Delta_{\Psi_{\star}}\right) \\
& \int_{0}^{v}\left(\left\|\nabla^{3} \rho\right\|_{L^{2}\left(\mathcal{S}_{u, v^{\prime}}\right)}+\left\|\nabla^{3} \sigma\right\|_{L^{2}\left(\mathcal{S}_{u, v^{\prime}}\right)}\right) \mathrm{d} v^{\prime} .
\end{aligned}
$$

The second representative term in the expansion of $\left\|\nabla^{3} \rho\right\|_{L^{2}\left(\mathcal{S}_{u, v}\right)}$ is $\left\|\varpi \delta^{2} \rho\right\|_{L^{2}\left(S_{\left.\mathcal{S}_{u, v}\right)}\right.}$ (recall that $\varpi \equiv \beta-\bar{\alpha}$ ). One has

$$
\begin{aligned}
D\left(\varpi \delta^{2} \rho\right) & =D \varpi\left(\delta^{2} \rho\right)+\varpi D \delta^{2} \rho \\
& =\left(\Psi_{1}+\Gamma^{2}+\delta \epsilon-\delta \bar{\epsilon}\right) \delta^{2} \rho+\Gamma^{5}+\Gamma^{3} \delta \Gamma+\varpi(\delta \Gamma)^{2}+\Gamma^{2} \delta^{2} \Gamma,
\end{aligned}
$$

from which we can conclude that

$$
\left\|\varpi \delta^{2} \rho\right\|_{L^{2}\left(\mathcal{S}_{u, v}\right)} \leq C\left(I, \Delta_{e_{\star}}, \Delta_{\Gamma_{\star}}, \Delta_{\Psi_{\star}}\right)+C\left(I, \Delta_{e_{\star}}, \Delta_{\Gamma_{\star}}, \Delta_{\Psi_{\star}}\right) \int_{0}^{v}\left\|\not^{3} \rho\right\|_{L^{2}\left(\mathcal{S}_{u, v^{\prime}}\right)} \mathrm{d} v^{\prime},
$$

by Sobolev embedding as before. The third representative term is $\|\delta \varpi \delta \rho\|_{L^{2}\left(\mathcal{S}_{u, v}\right)}$ for which we have

$$
D(\delta \varpi \delta \rho)=-\Psi_{1} \bar{\pi} \delta \rho+\Gamma^{3} \delta \rho+\delta \Psi_{1} \delta \rho+\Gamma(\delta \Gamma)^{2}+\delta^{2}(\epsilon-\bar{\epsilon}) \delta \rho,
$$

so that

$$
\|\delta \varpi \delta \rho\|_{L^{2}\left(\mathcal{S}_{u, v}\right)} \leq C\left(I, \Delta_{e_{\star}}, \Delta_{\Gamma_{\star}}, \Delta_{\Psi_{\star}}\right)+C\left(I, \Delta_{e_{\star}}, \Delta_{\Gamma_{\star}}, \Delta_{\Psi_{\star}}\right) \int_{0}^{v}\left\|\nabla^{3} \rho\right\|_{L^{2}\left(\mathcal{S}_{u, v^{\prime}}\right)} \mathrm{d} v^{\prime} .
$$

The fourth representative term is $\varpi^{2} \delta \rho$ for which we can compute

$$
D\left(\varpi^{2} \delta \rho\right)=2 \varpi \Psi_{1} \delta \rho+\Gamma^{3} \delta \Gamma+\Gamma(\delta \Gamma)^{2}+\Gamma^{5} .
$$

Consequently, one finds that

$$
\left\|\varpi^{2} \delta \rho\right\|_{L^{2}\left(\mathcal{S}_{u, v}\right)} \leq C\left(I, \Delta_{e_{\star}}, \Delta_{\Gamma_{\star}}, \Delta_{\Psi_{\star}}\right) .
$$

Combining all the expressions arising in the expansion of $\not^{3} \rho$ one then concludes,

$$
\begin{gathered}
\left\|\not \nabla^{3} \rho\right\|_{L^{2}\left(\mathcal{S}_{u, v}\right)} \leq C\left(I, \Delta_{e_{\star}}, \Delta_{\Gamma_{\star}}, \Delta_{\Psi_{\star}}\right)+C\left(I, \Delta_{e_{\star}}, \Delta_{\Gamma_{\star}}, \Delta_{\Psi_{\star}}\right) \\
\quad \int_{0}^{v}\left(\left\|\not \nabla^{3} \rho\right\|_{L^{2}\left(\mathcal{S}_{u, v^{\prime}}\right)}+\left\|\not \nabla^{3} \sigma\right\|_{L^{2}\left(\mathcal{S}_{u, v^{\prime}}\right)}\right) \mathrm{d} v^{\prime}
\end{gathered}
$$

and Grönwall's inequality finally gives

$$
\left\|\not \nabla^{3} \rho\right\|_{L^{2}\left(\mathcal{S}_{u, v}\right)} \leq C\left(I, \Delta_{e_{\star}}, \Delta_{\Gamma_{\star}}, \Delta_{\Psi_{\star}}\right)+C\left(I, \Delta_{e_{\star}}, \Delta_{\Gamma_{\star}}, \Delta_{\Psi_{\star}}\right) \int_{0}^{v}\left\|\not \nabla^{3} \sigma\right\|_{L^{2}\left(\mathcal{S}_{u, v^{\prime}}\right)} \mathrm{d} v^{\prime}
$$


In order to estimate $\left\|\not \nabla^{3} \sigma\right\|_{L^{2}\left(\mathcal{S}_{u, v}\right)}$, we make use, again, of the general expressions contained in "Appendix C". For brevity we focus our attention on $\left\|\delta^{3} \sigma\right\|_{L^{2}\left(\mathcal{S}_{u, v}\right)}$. Making use of the integration identity in "Appendix D" and the commutators one finds that

$$
\left\|\delta^{3} \sigma\right\|_{L^{2}\left(\mathcal{S}_{u, v}\right)}=\left\|\bar{\delta} \delta^{2} \sigma\right\|_{L^{2}\left(\mathcal{S}_{u, v}\right)}=\left\|\delta^{2} \bar{\delta} \sigma\right\|_{L^{2}\left(\mathcal{S}_{u, v}\right)}+\cdots
$$

where the ellipsis denotes lower order derivative terms. Now, the constraint structure equation (Codazzi equation) (30q) lets us transform this norm further to a norm of the same order for $\rho$. Thus, one concludes that

$$
\left\|\nabla^{3} \rho\right\|_{L^{2}\left(\mathcal{S}_{u, v}\right)} \leq C\left(I, \Delta_{e_{\star}}, \Delta_{\Gamma_{\star}}, \Delta_{\Psi_{\star}}\right)+C\left(I, \Delta_{e_{\star}}, \Delta_{\Gamma_{\star}}, \Delta_{\Psi_{\star}}\right) \int_{0}^{v}\left\|\nabla^{3} \rho\right\|_{L^{2}\left(\mathcal{S}_{u, v^{\prime}}\right)} \mathrm{d} v^{\prime}
$$

This inequality in turn implies that

$$
\begin{aligned}
& \left\|\nabla^{3} \rho\right\|_{L^{2}\left(\mathcal{S}_{u, v}\right)} \leq C\left(I, \Delta_{e_{\star}}, \Delta_{\Gamma_{\star}}, \Delta_{\Psi_{\star}}\right), \\
& \left\|\nabla^{3} \sigma\right\|_{L^{2}\left(\mathcal{S}_{u, v}\right)} \leq C\left(I, \Delta_{e_{\star}}, \Delta_{\Gamma_{\star}}, \Delta_{\Psi_{\star}}\right) .
\end{aligned}
$$

Estimates for $\tau$ and $\chi$ Making use of the structure Eq. (30b) and the commutators we obtain

$$
\begin{aligned}
D \delta^{3} \tau= & \delta^{3} \Psi_{1}+\Gamma \delta^{3} \Gamma_{1}+\Gamma \delta^{3} \tau+\Gamma \delta^{2} \Psi_{1}+\delta \Gamma \delta^{2} \Gamma+\Gamma^{2} \delta^{2} \Gamma \\
& +\Gamma^{2} \delta \Psi_{1}+\delta \Gamma \delta \Psi_{1}+\Gamma^{3} \delta \Gamma+\Gamma(\delta \Gamma)^{2}
\end{aligned}
$$

where $\Gamma_{1}$ contains combinations of $\epsilon, \alpha, \beta, \rho$ and $\sigma$. Thus, using the main bootstrap assumption and the definition of $\Delta_{\Psi}$ we obtain that

$$
\begin{aligned}
\left\|\not \nabla^{3} \tau\right\|_{L^{2}\left(\mathcal{S}_{u, v}\right) \leq} & C\left(I, \Delta_{e_{\star}}, \Delta_{\Gamma_{\star}}, \Delta_{\Psi_{\star}}, \Delta_{\Psi}\right)+C\left(I, \Delta_{e_{\star}}, \Delta_{\Gamma_{\star}}, \Delta_{\Psi_{\star}}\right) \\
& \int_{0}^{v}\left\|\nabla^{3} \tau\right\|_{L^{2}\left(\mathcal{S}_{u, v^{\prime}}\right)} \mathrm{d} v^{\prime} .
\end{aligned}
$$

Accordingly, using Grönwall's inequality one arrives to

$$
\left\|\not \nabla^{3} \tau\right\|_{L^{2}\left(\mathcal{S}_{u, v}\right)} \leq C\left(I, \Delta_{e_{\star}}, \Delta_{\Gamma_{\star}}, \Delta_{\Psi_{\star}}, \Delta_{\Psi}\right) .
$$

The construction of an estimate for $\chi$ is similar. In this case we obtain that

$$
\left\|\nabla^{3} \chi\right\|_{L^{2}\left(\mathcal{S}_{u, v}\right)} \leq C\left(I, \Delta_{e_{\star}}, \Delta_{\Gamma_{\star}}, \Delta_{\Psi_{\star}}, \Delta_{\Psi}\right) .
$$

Estimates for the remaining connection coefficients In order to provide estimates for

$$
\left\|\not \mathbb{X}^{3}\{\mu, \lambda, \alpha, \beta, \epsilon\}\right\|_{L^{2}\left(\mathcal{S}_{u, v}\right)},
$$


we make use of the transport equations along the short direction. The proofs for the various coefficients are similar so for brevity we discuss only the argument for $\epsilon$. In this case one can readily compute that

$$
\begin{aligned}
\Delta \delta^{3} \epsilon= & -\delta^{3} \Psi_{2}+\Gamma \delta^{3} \Gamma_{1}+\Gamma \delta^{3} \epsilon+\Psi_{1} \delta^{2} \Gamma+\delta \Gamma \delta^{2} \Gamma+\Gamma^{2} \delta^{2} \Gamma \\
& +\Gamma \delta^{2} \Psi_{2}+\Gamma^{2} \delta \Psi_{2}+\Gamma^{3} \delta \Gamma+\Gamma(\delta \Gamma)^{2}+\Gamma^{3} \Psi_{2}+\Gamma^{5}
\end{aligned}
$$

where the coefficients $\Gamma_{1}$ do not contain $\epsilon$. Making use of the short direction inequality of Proposition 2 we obtain that

$$
\begin{aligned}
\left\|\nabla^{3} \epsilon\right\|_{L^{2}\left(\mathcal{S}_{u, v}\right) \leq} & 2\left\|\nabla^{3} \epsilon\right\|_{L^{2}\left(\mathcal{S}_{0, v}\right)}+C\left(I, \Delta_{e_{\star}}, \Delta_{\Gamma_{\star}}, \Delta_{\Psi_{\star}}\right) \Delta_{\Psi} \varepsilon^{1 / 2} \\
& +C\left(I, \Delta_{e_{\star}}, \Delta_{\Gamma_{\star}}, \Delta \Psi_{\star}\right) \int_{0}^{u}\left\|\nabla^{3} \epsilon\right\|_{L^{2}\left(\mathcal{S}_{u^{\prime}, v}\right)} \mathrm{d} u^{\prime} .
\end{aligned}
$$

In particular, we can choose the range of integration sufficiently small so that

$$
\left\|\not \nabla^{3} \epsilon\right\|_{L^{2}\left(\mathcal{S}_{u, v}\right)} \leq 3 \Delta_{\Gamma_{\star}} .
$$

The argument for $\left\|\nabla^{3}\{\mu, \lambda, \alpha, \beta\}\right\|_{L^{2}\left(\mathcal{S}_{u, v}\right)}$ is the same.

Concluding the argument An inspection of the estimates obtained in the previous paragraphs shows that we have improved the initial bootstrap assumption. This concludes the proof of the proposition.

\subsection{Main estimates for the curvature}

We are now in the position to obtain the main estimates for the components of the Weyl tensor. We start with an estimate on a given pair of null hypersurfaces in terms of their value at hypersurfaces in the past.

Proposition 13 (Basic control of components of the Weyl tensor on the light cones in terms of its values on causal diamonds) Suppose that we are given a solution to the vacuum EFEs in Stewart's gauge and that $\mathcal{D}_{u, v}$ is contained in the existence area. The following $L^{2}$ estimates for the Weyl curvature hold:

$$
\begin{aligned}
& \sum_{i=0,1,2} \int_{\mathcal{N}_{u}(0, v)}\left|\Psi_{i}\right|^{2}+\sum_{j=1,2,3} \int_{\mathcal{N}_{v}^{\prime}(0, u)} Q^{-1}\left|\Psi_{j}\right|^{2} \\
& \quad \leq \sum_{i=0,1,2} \int_{\mathcal{N}_{0}(0, v)}\left|\Psi_{i}\right|^{2}+\sum_{j=1,2,3} \int_{\mathcal{N}_{0}(0, u)} Q^{-1}\left|\Psi_{j}\right|^{2}+\int_{\mathcal{D}_{u, v}}\left|\Psi_{H} \Psi \Gamma+c c\right|,
\end{aligned}
$$

where $\Psi$ contains $\Psi_{k}, k=0, \ldots, 4, \Psi_{H}$ denotes the components $\Psi_{k}, k=0, \ldots, 3$, "cc" denotes the complex conjugate of the last term on the right-hand side and $\Gamma$ stands for arbitrary connection coefficients from the collection $\{\mu, \lambda, \alpha, \beta, \epsilon, \rho, \sigma, \tau\}$. 
Proof Assuming, as always that the vacuum field equations of GR are satisfied, we start considering the Bianchi identities (31b) and (31a) written schematically as

$$
\begin{aligned}
& \Delta \Psi_{0}=\delta \Psi_{1}+\Gamma \Psi, \\
& D \Psi_{1}=\bar{\delta} \Psi_{0}+\Gamma \Psi .
\end{aligned}
$$

Then, integration by parts one obtains (again, using schematic notation) that

$$
\begin{aligned}
\int_{\mathcal{D}_{u, v}} \bar{\Psi}_{0} \Delta \Psi_{0} & =\int_{\mathcal{D}_{u, v}} \bar{\Psi}_{0} \delta \Psi_{1}+\int_{\mathcal{D}_{u, v}} \bar{\Psi}_{0} \Gamma \Psi \\
& =-\int_{\mathcal{D}_{u, v}} \Psi_{1} \delta \bar{\Psi}_{0}-\int_{\mathcal{D}_{u, v}} \Psi_{1} \bar{\Psi}_{0} \varpi+\int \bar{\Psi}_{0} \Gamma \Psi \\
& =-\int_{\mathcal{D}_{u, v}} \Psi_{1} D \bar{\Psi}_{1}+\int_{\mathcal{D}_{u, v}}\left\{\bar{\Psi}_{0}, \Psi_{1}\right\} \Gamma \Psi .
\end{aligned}
$$

Hence, using the identities in Lemma 8, we conclude that

$$
\begin{aligned}
\int_{\mathcal{N}_{u}(0, v)}\left|\Psi_{0}\right|^{2}+\int_{\mathcal{N}_{v}(0, u)} Q^{-1}\left|\Psi_{1}\right|^{2} \leq & \int_{\mathcal{N}_{0}(0, v)}\left|\Psi_{0}\right|^{2}+\int_{\mathcal{N}_{0}(0, u)} Q^{-1}\left|\Psi_{1}\right|^{2} \\
& +\int_{\mathcal{D}_{u, v}}\left(\left|\left\{\Psi_{0}, \Psi_{1}\right\} \Psi \Gamma+\mathrm{cc}\right|\right),
\end{aligned}
$$

where in the previous expression $\Psi$ contains $\Psi_{0,1,2}$. Analogous inequalities can be obtained for the pairs $\Delta \Psi_{1}, D \Psi_{2}$, and $\Delta \Psi_{2}, D \Psi_{3}$.

Similar estimates can be obtained for the first angular derivatives of the components of the Weyl tensor.

Proposition 14 (Control of the first angular derivatives of the components of the Weyl tensor) Again let $\mathcal{D}_{u, v}$ be contained in the existence area, then we have that

$$
\begin{aligned}
& \sum_{i=0,1,2} \int_{\mathcal{N}_{u}(0, v)}\left|\not \nabla \Psi_{i}\right|^{2}+\sum_{j=1,2,3} \int_{\mathcal{N}_{v}(0, u)} Q^{-1}\left|\not \nabla \Psi_{j}\right|^{2} \\
& \leq \sum_{i=0,1,2} \int_{\mathcal{N}_{0}(0, v)}\left|\not \nabla \Psi_{i}\right|^{2}+\sum_{j=1,2,3} \int_{\mathcal{N}_{0}(0, u)} Q^{-1}\left|\not \nabla \Psi_{j}\right|^{2} \\
& \quad+\int_{\mathcal{D}_{u, v}}\left|\not \nabla \Psi_{H}\right|\left(\left|\Psi \Gamma^{2}\right|+|\Gamma \not \nabla \Psi|+|\Psi \not \Gamma|\right),
\end{aligned}
$$

where $\Psi$ contains $\Psi_{k}, k=0, \ldots, 4$, and $\Psi_{H}$ contains $\Psi_{k}, k=0, \ldots, 3$, and again $\Gamma$ stands for some combination of the connection coefficients $\{\mu, \lambda, \alpha, \beta, \epsilon, \rho, \sigma, \tau\}$.

Proof Again, we make use of integration by parts. Consider for example

$$
\int_{\mathcal{D}_{u, v}} \bar{\delta} \bar{\Psi}_{0} \Delta \delta \Psi_{0}=\int_{\mathcal{D}_{u, v}} \bar{\delta} \bar{\Psi}_{0} \delta^{2} \Psi_{1}+\int_{\mathcal{D}_{u, v}} \bar{\delta} \bar{\Psi}_{0}\left(\Gamma^{2} \Psi_{i}+\Gamma \delta \Psi_{i}+\Psi_{i} \delta \Gamma\right)
$$




$$
\begin{aligned}
& =-\int_{\mathcal{D}_{u, v}} \delta \bar{\delta} \bar{\Psi}_{0} \delta \Psi_{1}+\int_{\mathcal{D}_{u, v}} \bar{\delta} \bar{\Psi}_{0}\left(\Gamma^{2} \Psi_{i}+\Gamma \delta \Psi_{i}+\Psi_{i} \delta \Gamma\right) \\
& =-\int_{\mathcal{D}_{u, v}} \delta \Psi_{1} D \bar{\delta} \bar{\Psi}_{1}+\int_{\mathcal{D}_{u, v}}\left(\bar{\delta} \bar{\Psi}_{0}, \delta \Psi_{1}\right)\left(\Gamma^{2} \Psi_{i}+\Gamma \delta \Psi_{i}+\Psi_{i} \delta \Gamma\right)
\end{aligned}
$$

with $i=0,1,2$. A similar expression can be obtained for the combination

$$
\int_{\mathcal{D}_{u, v}} \delta \bar{\Psi}_{0} \Delta \bar{\delta} \Psi_{0}+\int_{\mathcal{D}_{u, v}} \bar{\delta} \Psi_{1} D \delta \bar{\Psi}_{1}
$$

Thus, using Lemma 8 can conclude that

$$
\begin{aligned}
\int_{\mathcal{N}_{u}(0, v)}\left|\not \nabla \Psi_{0}\right|^{2}+\int_{\mathcal{N}_{v}(0, u)} Q^{-1}\left|\not \nabla \Psi_{1}\right|^{2} \leq & \int_{\mathcal{N}_{0}(0, v)}\left|\not \nabla \Psi_{0}\right|^{2}+\int_{\mathcal{N}_{0}(0, v)} Q^{-1}\left|\not \nabla \Psi_{1}\right|^{2} \\
& +\int_{\mathcal{D}_{u, v}}\left|\not \nabla\left\{\Psi_{0}, \Psi_{1}\right\}\right|\left(\left|\Psi \Gamma^{2}\right|+|\Gamma \not \nabla \Psi|+|\Psi \not \nabla \Gamma|\right),
\end{aligned}
$$

where $\Psi$ contains the components $\Psi_{0}, \Psi_{1}$ and $\Psi_{2}$. A similar computation for the other pairs of components renders the desired result.

The previous result can be extended to include higher order derivatives. More precisely:

Proposition 15 (Control of the higher angular derivatives of the components of the Weyl tensor) Let $\mathcal{D}_{u, v}$ again be contained in the existence area. Given a non-negative integer $m$, one has

$$
\begin{aligned}
\sum_{i=0,1,2} & \int_{\mathcal{N}_{u}(0, v)}\left|\nabla^{m} \Psi_{i}\right|^{2}+\sum_{j=1,2,3} \int_{\mathcal{N}_{v}(0, u)} Q^{-1}\left|\nabla^{m} \Psi_{j}\right|^{2} \\
\leq & \sum_{i=0,1,2} \int_{\mathcal{N}_{0}(0, v)}\left|\nabla^{m} \Psi_{i}\right|^{2}+\sum_{j=1,2,3} \int_{\mathcal{N}_{0}(0, v)} Q^{-1}\left|\nabla^{m} \Psi_{j}\right|^{2} \\
& +\int_{\mathcal{D}_{u, v}}\left|\nabla^{m} \Psi_{H}\right| \sum_{i_{1}+i_{2}+i_{3}+i_{4}=m}\left|\nabla^{i_{1}} \Gamma^{i_{2}}\right|\left|\nabla^{i_{3}} \Gamma\right|\left|\nabla^{i_{4}} \Psi\right| .
\end{aligned}
$$

where $\Psi$ contains the components $\Psi_{k}, k=0, \ldots, 4$, and $\Psi_{H}$ contains the components $\Psi_{k}, k=0, \ldots, 3$. Again $\Gamma$ stands for some combination of the connection coefficients $\{\mu, \lambda, \alpha, \beta, \epsilon, \rho, \sigma, \tau\}$. 
To wrap up the argument we also need estimates on the components $\Psi_{3}$ and $\Psi_{4}$. These follow from the Bianchi identities

$$
\begin{aligned}
& \Delta \Psi_{3}-\delta \Psi_{4}=4 \Psi_{4} \beta-\Psi_{4} \tau-4 \Psi_{3} \mu, \\
& D \Psi_{4}-\bar{\delta} \Psi_{3}=\Psi_{4}(\rho-4 \epsilon)+2 \Psi_{3}(3 \alpha+2 \beta)-3 \Psi_{2} \lambda .
\end{aligned}
$$

Using a similar approach to the one used in the previous propositions one can prove the following:

Proposition 16 (Control of the higher angular derivatives of the "bad" components of the Weyl tensor) Let $\mathcal{D}_{u, v}$ be contained in the existence area. Given a non-negative integer $m$, one has that

$$
\begin{aligned}
\int_{\mathcal{N}_{u}(0, v)}\left|\nabla^{m} \Psi_{3}\right|^{2}+ & \int_{\mathcal{N}_{v}(0, u)} Q^{-1}\left|\nabla^{m} \Psi_{4}\right|^{2} \\
\leq & \int_{\mathcal{N}_{0}(0, v)}\left|\nabla^{m} \Psi_{3}\right|^{2}+\int_{\mathcal{N}_{0}(0, u)} Q^{-1}\left|\nabla^{m} \Psi_{4}\right|^{2} \\
& +\int_{\mathcal{D}_{u, v}}\left|\nabla^{m} \Psi_{4}\right| \sum_{i_{1}+i_{2}+i_{3}+i_{4}=m}\left|\nabla^{i_{1}} \Gamma^{i_{2}}\right|\left|\nabla^{i_{3}} \Gamma^{\prime}\right|\left|\nabla^{i_{4}} \Psi_{4}\right| \\
& +\int_{\mathcal{D}_{u, v}}\left|\nabla^{m} \Psi_{3}\right| \sum_{i_{1}+i_{2}+i_{3}+i_{4}=m}\left|\nabla^{i_{1}} \Gamma^{i_{2}}\right|\left|\nabla^{i_{3}} \Gamma\right|\left|\nabla^{i_{4}} \Psi\right| \\
& +\int_{\mathcal{D}_{u, v}}\left|\nabla^{m} \Psi_{4}\right| \sum_{i_{1}+i_{2}+i_{3}+i_{4}=m}\left|\nabla^{i_{1}} \Gamma^{i_{2}}\right|\left|\nabla^{i_{3}} \Gamma\right|\left|\nabla^{i_{4}} \Psi_{H}^{\prime}\right|,
\end{aligned}
$$

where $\Psi$ contains the components $\Psi_{3}$ and $\Psi_{4}$, while $\Psi_{H}^{\prime}$ contains the components $\Psi_{2}$ and $\Psi_{3}$. Here $\Gamma$ stands for some combination of the connection coefficients $\{\mu, \lambda, \alpha, \beta, \epsilon, \rho, \tau, \sigma\}$. Because neither the coefficient of $\Psi_{4}$ on the right hand side of (24) nor the NP $\delta \bar{\delta}$-commutator (29d) contain $\tau, \chi$ terms, neither does $\Gamma^{\prime}$.

Propositions 13-16 clearly make no use of the estimates demonstrated in the previous sections. Finally, we therefore conclude this section with the main estimate for the components of the Weyl tensor employing our earlier work. This proposition makes only assumptions on the initial data.

Proposition 17 (Control of the components of the Weyl tensor in terms of the initial data) Suppose we are given a solution to the vacuum EFE's in Stewart's gauge emanating from data for the CIVP as prepared in Lemma 2, satisfying

$$
\Delta_{e_{\star}}, \Delta_{\Gamma_{\star}}, \Delta_{\Psi_{\star}}<\infty
$$

with the solution itself satisfying

$\left.\sup _{u, v}\|\{\mu, \lambda, \alpha, \beta, \epsilon, \rho, \sigma, \tau, \chi\}\|_{L^{\infty}\left(\mathcal{S}_{u, v}\right)}<\infty, \sup _{u, v} \| \not \mid \mu \mu, \lambda, \alpha, \beta, \epsilon, \rho, \sigma\right\} \|_{L^{4}\left(\mathcal{S}_{u, v}\right)}<\infty$, 


$$
\begin{aligned}
\sup _{u, v} & \left\|\nabla^{2}\{\mu, \lambda, \alpha, \beta, \epsilon, \rho, \sigma, \tau\}\right\|_{L^{2}\left(\mathcal{S}_{u, v}\right)}<\infty, \quad \sup _{u, v}\left\|\nabla^{3}\{\mu, \lambda, \alpha, \beta, \epsilon, \tau\}\right\|_{L^{2}\left(\mathcal{S}_{u, v}\right)}<\infty, \\
& \Delta_{\Psi}(\mathcal{S})<\infty, \quad \Delta_{\Psi}<\infty
\end{aligned}
$$

on some truncated causal diamond $\mathcal{D}_{u, v_{\bullet}}^{t}$. Then there exists $\varepsilon_{\star}=\varepsilon_{\star}\left(I, \Delta_{e_{\star}}, \Delta_{\Gamma_{\star}}, \Delta_{\Psi_{\star}}\right)$ such that for $\varepsilon_{\star} \leq \varepsilon$ we have

$$
\Delta_{\Psi} \leq C\left(I, \Delta_{e_{\star}}, \Delta_{\Gamma_{\star}}, \Delta_{\Psi_{\star}}\right) .
$$

Proof The aim in this proof is to control the terms involving integrals on the diamond $\mathcal{D}_{u, v}$ arising in Propositions 15 and 16 for $m \leq 3$. Starting with Proposition 15 one has that the relevant integral is given by

$$
\int_{\mathcal{D}_{u, v}}\left|\nabla^{m} \Psi_{H}\right| \sum_{i_{1}+i_{2}+i_{3}+i_{4}=m}\left|\nabla^{i_{1}} \Gamma^{i_{2}}\right|\left|\nabla^{i_{3}} \Gamma\right|\left|\nabla^{i_{4}} \Psi\right|,
$$

for $(u, v)$ in $\mathcal{D}_{\varepsilon, v_{\bullet}}^{t}$. On the one hand, for the first factor in this integral, given that $\Psi_{H} \in$ $\left\{\Psi_{0}, \Psi_{1}, \Psi_{2}, \Psi_{3}\right\}$ can be controlled in $L^{2}\left(\mathcal{N}_{u}(0, v)\right)$, one readily obtains

$$
\left\|\not \nabla^{m} \Psi_{H}\right\|_{L^{2}\left(\mathcal{D}_{u, v}\right)}=\left(\int_{0}^{u} \int_{0}^{v} \int_{\mathcal{S}_{u^{\prime}, v^{\prime}}}\left|\nabla^{m} \Psi_{H}\right|^{2} \mathrm{~d} v^{\prime} \mathrm{d} u^{\prime}\right)^{1 / 2} \leq C \Delta \Psi \varepsilon^{1 / 2}
$$

On the other, for the factors contains $\Psi_{4}$, one only has control on $\mathcal{N}_{v}(0, u)$ - that is,

$$
\left\|\nabla^{m} \Psi\right\|_{L^{2}\left(\mathcal{D}_{u, v}\right)} \leq C \Delta \Psi
$$

It then follows that the integral (25) can be estimated as,

$$
\begin{aligned}
& \int_{\mathcal{D}_{u, v}}\left|\nabla^{m} \Psi_{H}\right| \sum_{i_{1}+i_{2}+i_{3}+i_{4}=m}\left|\nabla^{i_{1}} \Gamma^{i_{2}} \| \nabla^{i_{3}} \Gamma\right|\left|\nabla^{i_{4}} \Psi\right| \\
& \leq C \varepsilon^{1 / 2} \Delta_{\Psi} \sum_{i_{1}+i_{2}+i_{3}+i_{4} \leq 3}\left\|\nabla^{i_{1}} \Gamma^{i_{2}} \not^{i_{3}} \Gamma \nabla^{i_{4}} \Psi\right\|_{L^{2}\left(\mathcal{D}_{u, v}\right)} .
\end{aligned}
$$

In particular, for $m=0$, the right-hand side of the above inequality gives

$$
\begin{aligned}
C \varepsilon^{1 / 2} \Delta_{\Psi}\|\Gamma \Psi\|_{L^{2}\left(\mathcal{D}_{u, v}\right)} & \leq C \epsilon^{1 / 2} \Delta_{\Psi}\|\Gamma\|_{L^{\infty}(S)}\|\Psi\|_{L^{2}\left(\mathcal{D}_{u, v}\right)} \\
& \leq C\left(I, \Delta_{e_{\star}}, \Delta_{\Gamma_{\star}}, \Delta_{\Psi_{\star}}, \Delta_{\Psi}\right) \varepsilon^{1 / 2} .
\end{aligned}
$$

Next, when $m=1$, we have that the right-hand of inequality (26) gives

$$
C \varepsilon^{1 / 2} \Delta_{\Psi}|| \Gamma^{2} \Psi+\left.\Gamma|\not| \Psi|+\Psi| \not \nabla \Gamma||\right|_{L^{2}\left(\mathcal{D}_{u, v}\right)} .
$$


The first two terms can be controlled like the case $m=0$, and the third can be controlled by means of Sobolev embedding:

$$
\begin{aligned}
\|\Psi|\not \nabla \Gamma|\|_{L^{2}\left(\mathcal{S}_{u, v}\right)} & \leq\|\not \nabla \Gamma\|_{L^{\infty}\left(\mathcal{S}_{u, v}\right)}\|\Psi\|_{L^{2}\left(\mathcal{D}_{u, v}\right)} \\
& \leq\left(\|\not \nabla \Gamma\|_{L^{2}\left(\mathcal{S}_{u, v}\right)}+\left\|\not \nabla^{2} \Gamma\right\|_{L^{2}\left(\mathcal{S}_{u, v}\right)}+\left\|\not \nabla^{3} \Gamma\right\|_{L^{2}\left(\mathcal{S}_{u, v}\right)}\right)\|\Psi\|_{L^{2}\left(\mathcal{D}_{u, v}\right)}
\end{aligned}
$$

For the case $m=2$, the terms on the right-hand side of inequality (26) give

$C \varepsilon^{1 / 2} \Delta_{\Psi}|| \Gamma\left|\not \nabla^{2} \Psi\right|+\Gamma^{3} \Psi+\Gamma^{2}|\not \nabla \Psi|+\Psi \Gamma|\not \nabla \Gamma|+|\not| \Psi|| \not \nabla \Gamma|+\Psi| \not{ }^{2} \Gamma||_{L^{2}\left(\mathcal{D}_{u, v}\right)}$.

All terms, save last one, can be controlled by analysis analogous to that used in the previous cases. To see this, we split the $L^{\infty}$-norm of the connection coefficient and the $L^{2}$-normal of the curvature. The $L^{\infty}$-normal can then be controlled by means of Sobolev embedding. For the last term, we have

$$
\begin{aligned}
\left(\int_{0}^{u} \int_{0}^{v} \int_{\mathcal{S}_{u^{\prime}, v^{\prime}}}\left(\Psi\left|\nabla^{2} \Gamma\right|\right)^{2} \mathrm{~d} v^{\prime} \mathrm{d} u^{\prime}\right)^{1 / 2} & \leq\left(\int_{0}^{u} \int_{0}^{v}\|\Psi\|_{L^{\infty}\left(\mathcal{S}_{u^{\prime}, v^{\prime}}\right)}^{2}\left\|\not \nabla^{2} \Gamma\right\|_{L^{2}\left(\mathcal{S}_{u^{\prime}, v^{\prime}}\right)}^{2} \mathrm{~d} v^{\prime} \mathrm{d} u^{\prime}\right)^{1 / 2} \\
& \leq\left(\sup _{\mathcal{D}_{u, v}}\left\|\not \nabla^{2} \Gamma\right\|_{L^{2}\left(\mathcal{S}_{u^{\prime}, v^{\prime}}\right)}\right) \sum_{i=0}^{2}\left\|\nabla^{i} \Psi\right\|_{L^{2}\left(\mathcal{D}_{u, v}\right)},
\end{aligned}
$$

hence (27) under control.

Finally, when $m=3$ the terms on the right-hand side of inequality (26) give

$$
\begin{aligned}
& C \varepsilon^{1 / 2} \Delta \Psi||\left(\Gamma\left|\nabla^{3} \Psi\right|+\Psi\left|\nabla^{3} \Gamma\right|+|\not| \Gamma|| \nabla^{2} \Psi|+| \not \nabla \Psi|| \nabla^{2} \Gamma\left|+\Gamma^{2}\right| \not{ }^{2} \Psi|+\Gamma \Psi| \not \nabla^{2} \Gamma \mid\right. \\
& \left.+\Gamma|\not \nabla \Gamma||\not \nabla \Psi|+\Psi|\not \Gamma|^{2}+\Gamma^{3}|\not \nabla \Psi|+\Psi \Gamma^{2}|\not| \Gamma \mid+\Gamma^{4} \Psi\right) \|_{L^{2}\left(\mathcal{D}_{u, v}\right)} .
\end{aligned}
$$

The various terms in this expression can be estimated in a manner analogous to the previous cases. We conclude that the integral over $\mathcal{D}_{u, v}$ can be controlled by

$$
\int_{\mathcal{D}_{u, v}}\left|\nabla^{m} \Psi_{H}\right| \sum_{i_{1}+i_{2}+i_{3}+i_{4}=m}\left|\nabla^{i_{1}} \Gamma^{i_{2}}\right|\left|\nabla^{i_{3}} \Gamma\right|\left|\nabla^{i_{4}} \Psi\right| \leq C\left(I, \Delta_{e_{\star}}, \Delta_{\Gamma_{\star}}, \Delta_{\Psi_{\star}}, \Delta_{\Psi}\right) \varepsilon^{1 / 2}
$$

We now proceed to examine the estimate from Proposition 16. The terms in

$$
\int_{\mathcal{D}_{u, v}}\left|\not \nabla^{m} \Psi_{3}\right| \sum_{i_{1}+i_{2}+i_{3}+i_{4}=m}\left|\nabla^{i_{1}} \Gamma^{i_{2}}\right|\left|\nabla^{i_{3}} \Gamma\right|\left|\nabla^{i_{4}} \Psi\right|
$$

are identical to those already analysed and can be controlled by

$$
C\left(I, \Delta_{e_{\star}}, \Delta_{\Gamma_{\star}}, \Delta_{\Psi_{\star}}, \Delta_{\Psi}\right) \varepsilon^{1 / 2} .
$$


The terms

$$
\int_{\mathcal{D}_{u, v}}\left|\nabla^{m} \Psi_{4}\right| \sum_{i_{1}+i_{2}+i_{3}+i_{4}=m}\left|\nabla^{i_{1}} \Gamma^{i_{2}}\right|\left|\nabla^{i_{3}} \Gamma\right|\left|\mathbb{X}^{i_{4}} \Psi_{H}^{\prime}\right|
$$

can also be controlled because the components of the Weyl tensor contained in $\Psi_{H}^{\prime}=$ $\left\{\Psi_{2}, \Psi_{3}\right\}$ have already been shown to be controlled. The remaining terms are

$$
\int_{\mathcal{D}_{u, v}}\left|\nabla^{m} \Psi_{4}\right| \sum_{i_{1}+i_{2}+i_{3}+i_{4}=m}\left|\nabla^{i_{1}} \Gamma^{\prime i_{2}}\right|\left|\nabla^{i_{3}}(\rho+\epsilon)\right|\left|\nabla^{i_{4}} \Psi_{4}\right|
$$

We proceed to by treating $m=0, \ldots, 3$ individually. Notice in particular, that $\Gamma^{\prime}$ does contains neither $\tau$ nor $\chi$. Crucially the weakest bounds of Proposition 11 and Proposition 12 involving $\Delta_{\Psi}$ are therefore not invoked in the resulting computation, and so after a lengthy analysis one concludes that these terms satisfy

$$
\begin{aligned}
& \int_{\mathcal{D}_{u, v}}\left|\nabla^{m} \Psi_{4}\right| \sum_{i_{1}+i_{2}+i_{3}+i_{4}=m}\left|\nabla^{i_{1}} \Gamma^{i_{2}}\right|\left|\nabla^{i_{3}}(\rho+\epsilon) \| \mathbb{X}^{i_{4}} \Psi_{4}\right| \\
& \leq C\left(I, \Delta_{e_{\star}}, \Delta_{\Gamma_{\star}}, \Delta_{\Psi_{\star}}\right) \int_{0}^{v}\left\|\nabla^{m} \Psi_{4}\right\|_{L^{2}\left(\mathcal{N}_{v^{\prime}}(0, u)\right)} \sum_{i=0}^{m}\left\|\nabla^{i} \Psi_{4}\right\|_{L^{2}\left(\mathcal{N}_{v^{\prime}}(0, u)\right)} \mathrm{d} v^{\prime} \\
& \leq C\left(I, \Delta_{e_{\star}}, \Delta_{\Gamma_{\star}}, \Delta_{\Psi_{\star}}\right) \int_{0}^{v} \sum_{i=0}^{m}\left\|\nabla^{i} \Psi_{4}\right\|_{L^{2}\left(\mathcal{N}_{v^{\prime}}(0, u)\right)}^{2} \mathrm{~d} v^{\prime} .
\end{aligned}
$$

Substituting the previous expressions into the inequality of Proposition 16 one concludes that

$$
\begin{aligned}
\sum_{i=0}^{3}\left\|\nabla^{i} \Psi_{4}\right\|_{L^{2}\left(\mathcal{N}_{v}(0, u)\right)}^{2} \leq & C \Delta_{\Psi_{\star}}+C\left(I, \Delta_{e_{\star}}, \Delta_{\Gamma_{\star}}, \Delta \Psi_{\star}, \Delta_{\Psi}\right) \varepsilon^{1 / 2} \\
& +C\left(I, \Delta_{e_{\star}}, \Delta_{\Gamma_{\star}}, \Delta_{\Psi_{\star}}\right) \int_{0}^{v} \sum_{i=0}^{m}\left\|\mathbb{X}^{i} \Psi_{4}\right\|_{L^{2}\left(\mathcal{N}_{v}(0, u)\right)}^{2} \mathrm{~d} v^{\prime} .
\end{aligned}
$$

Accordingly, using Grönwall's inequality and taking $\varepsilon$ sufficiently small one finds,

$$
\begin{aligned}
\sum_{i=0}^{3}\left\|\not \nabla^{i} \Psi_{4}\right\|_{L^{2}\left(\mathcal{N}_{v}(0, u)\right)}^{2} & \leq C \Delta_{\Psi_{\star}}+C\left(I, \Delta_{e_{\star}}, \Delta_{\Gamma_{\star}}, \Delta_{\Psi_{\star}}, \Delta_{\Psi}\right) \varepsilon^{1 / 2} \\
& \leq C\left(I, \Delta_{e_{\star}}, \Delta_{\Gamma_{\star}}, \Delta_{\Psi_{\star}}\right)
\end{aligned}
$$

Using this estimate, it follows that

$$
\Delta_{\Psi} \leq C\left(I, \Delta_{e_{\star}}, \Delta_{\Gamma_{\star}}, \Delta_{\Psi_{\star}}\right)+C\left(I, \Delta_{e_{\star}}, \Delta_{\Gamma_{\star}}, \Delta_{\Psi_{\star}}, \Delta_{\Psi}\right) \varepsilon^{1 / 2} .
$$


Taking $\varepsilon$ small enough we have proven the proposition.

\section{Last slice argument and the end of the proof}

In this section we make use of the estimates developed in the previous sections to show the existence of solutions to the vacuum Einstein field equations exists in the rectangular domain

$$
\mathcal{D}=\left\{0 \leq u \leq \varepsilon, 0 \leq v \leq v_{\bullet}\right\}
$$

The strategy makes use of an argument by contradiction known as the last slice argument, in which it is assumed that the solution does not fill the whole of $\mathcal{D}$ and, accordingly, there exists a hypersurface (the last slice) which bounds the domain of existence of the solution. The estimates we have constructed in the previous sections allow then to show that, in fact, on this slice the solution and its derivatives are bounded. Thus, it is possible to make use of the standard Cauchy problem for the Einstein field equations to show that the solution extends beyond the hypersurface $t^{*}$-an observation which contradicts the original assumption.

\subsection{Setup}

In order to implement the above strategy one foliates the rectangle $\mathcal{D}$ by means of spacelike hypersurfaces. To this end recall definition (5) of the time function

$$
t \equiv u+v
$$

so that $\nabla t$ is timelike. Let $\Sigma_{t}$ denote the level sets of $t$.

The last slice argument starts by invoking the local existence result for the CIVP based on Rendall's reduction strategy. This result ensures the existence of a solution to evolution equations in a neighbourhood $\mathcal{V}$ of $\mathcal{S}_{\star}$ on $J^{+}\left(\mathcal{S}_{\star}\right)$ - see Theorem 2. Within this neighbourhood there exists a truncated causal diamond on which all the bootstrap assumptions required to obtain the estimates from the previous sections hold. Thus, we know that the set on which the bootstrap hypotheses hold is non-empty, and hence render our estimates applicable. The rest of the last slice argument proceeds now to show that this basic truncated causal diamond can be progressively enlarged as long as one has control on the initial data on the null cone $\mathcal{N}_{\star}$ thus exhausting the domain $\mathcal{D}$.

If the solution does not exist in the whole of $\mathcal{D}$, we must have $t^{*} \in(0, I+\varepsilon)$ such that

$$
t^{*}=\sup \left\{t: \text { the spacetime exists in } \mathcal{D} \cap \cup_{\tau \in[0, t)} \Sigma_{\tau}\right\}
$$

Let $\boldsymbol{h}_{t}$ and $\boldsymbol{K}_{t}$ be, respectively, the induced metric and second fundamental form on $\Sigma_{t}$. A schematic depiction of the geometric set-up is shown in Fig. 3. 


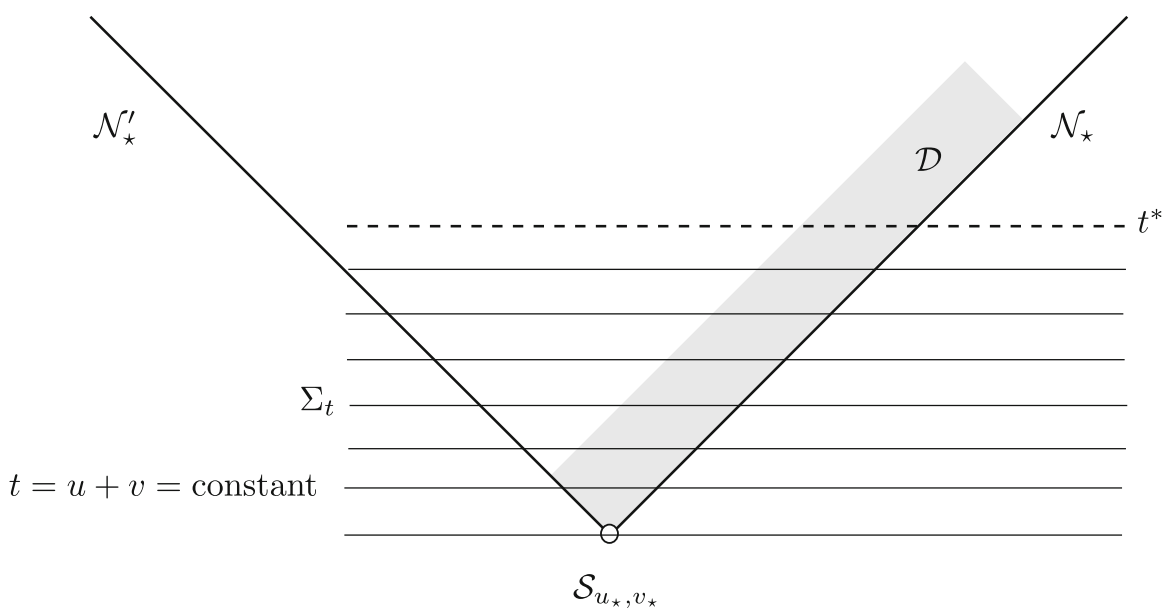

Fig. 3 Setup for the last slice argument. On each slice of the family of hypersurfaces $\Sigma_{t}$ one has a smooth initial data set $\left(\boldsymbol{h}_{t}, \boldsymbol{K}_{t}\right)$ for the vacuum Einstein field equations. The estimates of Proposition 17 then show that even on the last slice $\Sigma_{t^{*}}$ one has a well initial data set. Thus, the solution can be extended beyond this slice-a contradiction!

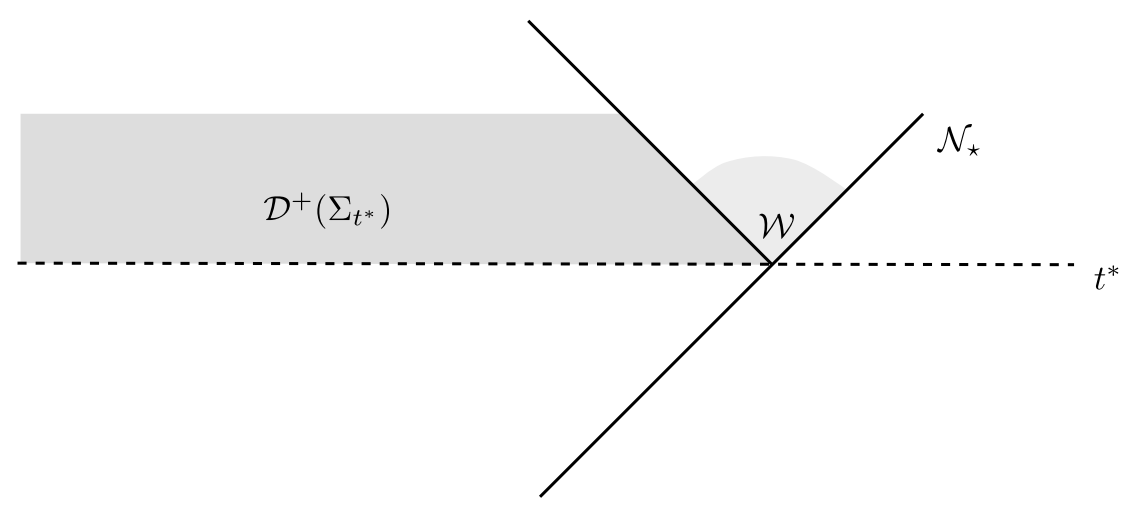

Fig. 4 Zoom in on the hypothetical last slice. Regular Cauchy initial data on $\Sigma_{t^{*}}$ allows to extend the solution to, at least, a slab on $\mathcal{D}^{+}\left(\Sigma_{t} *\right)$ making use of the standard Cauchy problem for the Einstein field equations. On the wedge $\mathcal{W}$, a solution can be recovered by appealing to Rendall's formulation of the local CIVP

\subsection{Main argument}

In the following we will show that the fields $\boldsymbol{h}_{t}$ and $\boldsymbol{K}_{t}$ converge in $C^{\infty}$ to fields $\boldsymbol{h}_{t^{*}}$ and $\boldsymbol{K}_{t^{*}}$. Moreover, it will be shown that the pair $\left(\boldsymbol{h}_{t^{*}}, \boldsymbol{K}_{t^{*}}\right)$ satisfy the Einstein constraint equations on $\Sigma_{t^{*}}$. In order to show this, it is necessary to show that all derivatives of $\boldsymbol{h}_{t}$ are bounded uniformly in $L^{2}\left(\Sigma_{t}\right)$ for all $t<t^{*}$. The method proceeds by induction:

Base step The first step corresponds, in essence, to the estimates obtained in the previous sections. More precisely, we have first derived uniform estimates for the $L^{\infty}$ norm of the zeroth order derivatives of connection on $\mathcal{D}$-see Proposition 7. For this 
we needed to assume that

$$
\sup _{u, v}\left\|\nabla^{2} \tau\right\|_{L^{2}\left(\mathcal{S}_{u, v}\right)}<\infty, \quad \sup _{u, v}\left\|\not \nabla^{3} \tau\right\|_{L^{2}\left(\mathcal{S}_{u, v}\right)}<\infty, \quad \Delta_{\Psi}(\mathcal{S})<\infty, \quad \Delta_{\Psi}<\infty
$$

on the truncated causal diamond. These conditions also lead to the analysis the $L^{4}$ norms of the first order derivatives (Proposition 8 ) and $L^{2}$-norms of the second order derivatives of the connection-see Proposition 9. Now, using the bootstrap assumptions, it follows that $\Delta_{\Psi}(\mathcal{S})<\infty$ uniformly on $\mathcal{D}$ with bounds given in terms of the initial data - thus, this condition can be removed from the list in (28). Similarly, we can also drop the condition $\left\|\nabla^{3} \tau\right\|_{L^{2}\left(\mathcal{S}_{u, v}\right)}<\infty$ and estimate the $L^{2}$-norm of the third order angular derivatives of the connection. In order to do so, we make use of the $D$ direction (i.e. the long direction) equations for the NP coefficients $\rho$ and $\sigma$, rather than the equations along the short direction as we want to avoid dealing with the higher order derivative of $\tau$ on spheres $\mathcal{S}_{u, v}$. Now, using integration by parts, one concludes that $\Delta_{\Psi}$ satisfies a similar uniform bound on $\mathcal{D}$. Thus, it has been shown that given some initial data on the initial light cone, it is possible to estimate the $L^{2}$-norm on the spheres $\mathcal{S}_{u, v}$ of the connection coefficients and their derivatives up to third order.

Intermediate step The previous analysis is the base step of the induction. As an intermediate induction step one analyses the fourth order derivatives of the connection coefficients. To this end, we make use of the same approach used in the analysis of the third order derivatives in Proposition 12 This approach requires the control of the norms of the fourth order derivatives of the components of the Weyl tensor on the light cone. As in the case of the Base Step, the required bounds need to be uniform on the truncated causal diamond with bounds given in terms of the initial data. This control can be achieved by the using integration by parts as in the analysis of Proposition 17.

Remark 17 The reason the method to analyse the fourth order derivatives of the connection coefficients is different from that of the third and lower orders lies in the structural properties of the equations - these properties become manifest when considering higher order derivatives. In particular, one has that:

(i) For zeroth-order derivatives, we cannot make use of the Codazzi equation to access the norms of $\rho$ and $\sigma$, since the Codazzi equation is a first order equation for the derivatives of $\rho$ and $\sigma$. Further difficulties arise from the nonlinear term $\rho^{2}$ in the $D$-direction Eq. (30m) for the coefficient $\rho$.

(ii) For the first-order derivatives, we can readily estimate the $L^{2}$-norm of the connection. However, this is not enough for the second order derivatives. In the $L^{2}$ estimate for the second order derivatives of the connections, we need Hölder's inequality to separate products of the form $\delta \Gamma \times \delta \Gamma$. This procedure leads to estimates involving the $L^{4}$-norm.

Induction step A procedure analogous to the one used to control the fourth order derivatives of the connection coefficients is employed to estimate the $k+1$-th order derivatives of the connection if control on the derivatives of $k$-th order is assumed. This calculation, requires, in particular, control of the value of such norms on the 
initial light cone- this control follows readily from the procedure used to evaluate the formal derivatives on the initial light cone-see Lemma 4.

Concluding the argument The previous step shows that it is possible to obtain control over the $L^{2}$-norms of all angular derivatives of the connection over the rectangular domain $\mathcal{D}$. Control of the derivatives respect to the optical functions $u$ and $v$ can be obtained by applying, as required, the directional covariant derivatives $D$ and $\Delta$ to the evolution equations and commuting. Since the domain is bounded, then all derivatives of $\boldsymbol{h}_{t}$ and $\boldsymbol{K}_{t}$ are bounded uniformly in $L^{2}\left(\Sigma_{t}\right)$ for $t<t^{*}$. Moreover, one has that the 1-parameter family of data $\left(\boldsymbol{h}_{t}, \boldsymbol{K}_{t}\right)$ converges uniformly in $C^{\infty}$ to a pair $\left(\boldsymbol{h}_{t^{*}}, \boldsymbol{K}_{t^{*}}\right)$. The pair $\left(\boldsymbol{h}_{t^{*}}, \boldsymbol{K}_{t^{*}}\right)$ satisfies the Einstein constraint equations on the hypersurface defined $t=t^{*}$-see [18]. This leads to a contradiction with the assumption of the existence of a last slice as the theory of the Cauchy problem for the Einstein field equations allows us to readily obtain a (future) development of the data set $\left(\boldsymbol{h}_{t^{*}}, \boldsymbol{K}_{t^{*}}\right)$ - see Fig. 4 Thus, no such last slice exists and the solution to the Einstein vacuum equations exists on the whole of the rectangular domain $\mathcal{D}$.

\subsection{Statement of the main result}

The long analysis of the preceding sections leads to the following:

Theorem 4 (Main result-improved local existence for the CIVP for the EFE) Given regular initial data for the vacuum Einstein field equations as contructed in Lemma 2 on the null hypersurfaces $\mathcal{N}_{\star} \cup \mathcal{N}_{\star}$ for $I \equiv\left\{0 \leq v \leq v_{\bullet}\right\}$, there exists $\varepsilon>0$ such that a unique smooth solution to the vacuum Einstein field equations exists in the region where $v \in I$ and $0 \leq u \leq \varepsilon$ defined by the null coordinates $(u, v)$. The number $\varepsilon$ can be chosen to depend only on $I, \Delta_{e_{\star}}, \Delta_{\Gamma_{\star}}$ and $\Delta_{\Psi_{\star}}$. Furthermore, in this area one has that,

$$
\begin{aligned}
& \sup _{u, v} \sup _{\Gamma \in\{\mu, \lambda, \rho, \sigma, \alpha, \beta, \epsilon, \tau, \chi\}} \max \left\{\sum_{i=0}^{1}\left\|\nabla^{i} \Gamma\right\|_{L^{\infty}\left(\mathcal{S}_{u, v}\right)}, \sum_{i=0}^{2}\left\|\not \nabla^{i} \Gamma\right\|_{L^{4}\left(\mathcal{S}_{u, v}\right)}, \sum_{i=0}^{3}\left\|\nabla^{i} \Gamma\right\|_{L^{2}\left(\mathcal{S}_{u, v}\right)}\right\} \\
& +\sum_{i=0}^{3} \sup _{\Psi \in\left\{\Psi_{0}, \Psi_{1}, \Psi_{2}, \Psi_{3}\right\}} \sup _{u}\left\|\not \nabla^{i} \Psi\right\|_{L^{2}\left(\mathcal{N}_{u}\right)}+\sup _{\Psi \in\left\{\Psi_{1}, \Psi_{2}, \Psi_{3}, \Psi_{4}\right\}} \sup _{v}\left\|\not^{i} \Psi\right\|_{L^{2}\left(\mathcal{N}_{v}\right)} \\
& \quad \leq C\left(I, \Delta_{e_{\star}}, \Delta_{\Gamma_{\star}}, \Delta_{\Psi_{\star}}\right) .
\end{aligned}
$$

Acknowledgements We are grateful to Edgar Gasperin and Arick Shao for helpful discussions. We also gratefully acknowledge support offered by the London Mathematical Society research in pairs scheme. DH was supported by the FCT (Portugal) IF Program IF/00577/2015 and PTDC/MAT-APL/30043/2017. PZ acknowledges the support of the China Scholarship Council.

Open Access This article is licensed under a Creative Commons Attribution 4.0 International License, which permits use, sharing, adaptation, distribution and reproduction in any medium or format, as long as you give appropriate credit to the original author(s) and the source, provide a link to the Creative Commons licence, and indicate if changes were made. The images or other third party material in this article are included in the article's Creative Commons licence, unless indicated otherwise in a credit line to the material. If material is not included in the article's Creative Commons licence and your intended use is not permitted by statutory regulation or exceeds the permitted use, you will need to obtain permission directly from the copyright holder. To view a copy of this licence, visit http://creativecommons.org/licenses/by/4.0/. 


\section{A: The Einstein field equations in the NP formalism}

This appendix serves as quick reference of the basic equations of our analysis. Throughout we make use of the NP formalism in the conventions used in the book by J. Stewart [26] which, in turn, follows the conventions in [27].

\section{The spin connection coefficients}

Given a NP frame $\left\{l^{a}, n^{a}, m^{a}, \bar{m}^{a}\right\}$, we define the complex spin connection coefficients as,

$$
\begin{aligned}
& \kappa \equiv-m^{a} l^{b} \nabla_{b} l_{a}, \quad \rho \equiv-m^{a} \bar{m}^{b} \nabla_{b} l_{a}, \quad \sigma \equiv-m^{a} m^{b} \nabla_{b} l_{a}, \quad \tau \equiv-m^{a} n^{b} \nabla_{b} l_{a}, \\
& v \equiv \bar{m}^{a} n^{b} \nabla_{b} n_{a}, \quad \mu \equiv \bar{m}^{a} m^{b} \nabla_{b} n_{a}, \quad \lambda \equiv \bar{m}^{a} \bar{m}^{b} \nabla_{b} n_{a}, \quad \pi \equiv \bar{m}^{a} l^{b} \nabla_{b} n_{a}, \\
& \alpha \equiv \frac{1}{2}\left(l^{a} \bar{m}^{b} \nabla_{b} n_{a}-m^{a} \bar{m}^{b} \nabla_{b} \bar{m}_{a}\right), \quad \quad \beta \equiv \frac{1}{2}\left(\bar{m}^{a} m^{b} \nabla_{b} m_{a}-n^{a} m^{b} \nabla_{b} l_{a}\right), \\
& \epsilon \equiv \frac{1}{2}\left(\bar{m}^{a} l^{b} \nabla_{b} m_{a}-n^{a} l^{b} \nabla_{b} l_{a}\right), \quad \gamma \equiv \frac{1}{2}\left(l^{a} n^{b} \nabla_{b} n_{a}-m^{a} n^{b} \nabla_{b} \bar{m}_{a}\right) .
\end{aligned}
$$

\section{The directional covariant derivatives}

The directional covariant derivatives along the directions given by the elements of the NP frame are given by

$$
D \equiv l^{a} \nabla_{a}, \quad \Delta \equiv n^{a} \nabla_{a} \quad \delta \equiv m^{a} \nabla_{a}, \quad \bar{\delta}=\bar{m}^{a} \nabla_{a}
$$

\section{The commutators}

The NP directional covariant derivatives satisfy the commutator relations

$$
\begin{aligned}
(\Delta D-D \Delta) \psi & =((\gamma+\bar{\gamma}) D+(\epsilon+\bar{\epsilon}) \Delta-(\bar{\tau}+\pi) \delta-(\tau+\bar{\pi}) \bar{\delta}) \psi, \\
(\delta D-D \delta) \psi & =((\bar{\alpha}+\beta-\bar{\pi}) D+\kappa \Delta-(\bar{\rho}+\epsilon-\bar{\epsilon}) \delta-\sigma \bar{\delta}) \psi, \\
(\delta \Delta-\Delta \delta) \psi & =(-\bar{\nu} D+(\tau-\bar{\alpha}-\beta) \Delta+(\mu-\gamma+\bar{\gamma}) \delta+\bar{\lambda} \bar{\delta}) \psi, \\
(\bar{\delta} \delta-\delta \bar{\delta}) \psi & =((\bar{\mu}-\mu) D+(\bar{\rho}-\rho) \Delta+(\alpha-\bar{\beta}) \delta-(\bar{\alpha}-\beta) \bar{\delta}) \psi
\end{aligned}
$$

where $\psi$ is any scalar field. Here we have highlighted the terms which vanish in our gauge. 


\section{The components of the curvature}

The components of the Weyl tensor $C_{a b c d}$, trace-free Ricci tensor $\Phi_{a b}$ and the Ricci scalar $R$, namely $\left\{\Psi_{0}, \Psi_{1}, \Psi_{2}, \Psi_{3}, \Psi_{4}\right\},\left\{\Phi_{00}, \Phi_{01}, \Phi_{02}, \Phi_{11}, \Phi_{12}, \Phi_{22}\right\}$ and $\Lambda$ are defined as

$$
\begin{aligned}
& \Psi_{0} \equiv C_{a b c d} l^{a} m^{b} l^{c} m^{d}, \\
& \Psi_{1} \equiv C_{a b c d} l^{a} n^{b} l^{c} m^{d}, \\
& \Psi_{2} \equiv \frac{1}{2} C_{a b c d} l^{a} n^{b}\left(l^{c} n^{d}-m^{c} \bar{m}^{d}\right) \\
& \Psi_{3} \equiv C_{a b c d} n^{a} l^{b} n^{c} \bar{m}^{d}, \\
& \Psi_{4} \equiv C_{a b c d} n^{a} \bar{m}^{b} n^{c} \bar{m}^{d}, \\
& \Phi_{00} \equiv \frac{1}{2} R_{\{a b\}} l^{a} l^{b}, \\
& \Phi_{01} \equiv \frac{1}{2} R_{\{a b\}} l^{a} m^{b}, \\
& \Phi_{02} \equiv \frac{1}{2} R_{\{a b\}} m^{a} m^{b}, \\
& \Phi_{11} \equiv \frac{1}{4} R_{\{a b\}}\left(l^{a} n^{b}+m^{a} \bar{m}^{b}\right), \\
& \Phi_{12} \equiv \frac{1}{2} R_{\{a b\}} n^{a} m^{b}, \\
& \Phi_{22} \equiv \frac{1}{2} R_{\{a b\}} n^{a} n^{b}, \\
& \Lambda \equiv-\frac{R}{24}
\end{aligned}
$$

where the curly brackets denote the symmetric, trace-free part.

\section{A.0.1: The NP Ricci identities}

The NP Ricci identities (also known as the second structure equations) take the form:

$$
\begin{aligned}
\Delta \epsilon-D \gamma & =\Lambda-\Phi_{11}-\Psi_{2}+\epsilon(2 \gamma+\bar{\gamma})+\gamma \bar{\epsilon}+\kappa \nu-\beta \pi-\alpha \bar{\pi}-\alpha \tau-\pi \tau-\beta \bar{\tau} \\
\Delta \kappa-D \tau & =-\Phi_{01}-\Psi_{1}+3 \gamma \kappa+\bar{\gamma} \kappa-\bar{\pi} \rho-\pi \sigma-\epsilon \tau+\bar{\epsilon} \tau-\rho \tau-\sigma \bar{\tau} \\
\Delta \pi-D \nu & =-\Phi_{21}-\Psi_{3}+3 \epsilon \nu+\bar{\epsilon} \nu-\gamma \pi+\bar{\gamma} \pi-\mu \pi-\lambda \bar{\pi}-\lambda \tau-\mu \bar{\tau} \\
\delta \gamma-\Delta \beta & =\Phi_{12}-\bar{\alpha} \gamma-2 \beta \gamma+\beta \bar{\gamma}+\alpha \bar{\lambda}+\beta \mu-\epsilon \bar{v}-v \sigma+\gamma \tau+\mu \tau \\
\delta \epsilon-D \beta & =-\Psi_{1}+\bar{\alpha} \epsilon+\beta \bar{\epsilon}+\gamma \kappa+\kappa \mu-\epsilon \bar{\pi}-\beta \bar{\rho}-\alpha \sigma-\pi \sigma \\
\delta \kappa-D \sigma & =-\Psi_{0}+\bar{\alpha} \kappa+3 \beta \kappa-\kappa \bar{\pi}-3 \epsilon \sigma+\bar{\epsilon} \sigma-\rho \sigma-\bar{\rho} \sigma+\kappa \tau \\
\delta \nu-\Delta \mu & =\Phi_{22}+\lambda \bar{\lambda}+\gamma \mu+\bar{\gamma} \mu+\mu^{2}-\bar{\alpha} \nu-3 \beta \nu-\bar{\nu} \pi+\nu \tau \\
\delta \pi-D \mu & =-2 \Lambda-\Psi_{2}+\epsilon \mu+\bar{\epsilon} \mu+\kappa \nu+\bar{\alpha} \pi-\beta \pi-\pi \bar{\pi}-\mu \bar{\rho}-\lambda \sigma \\
\delta \tau-\Delta \sigma & =\Phi_{02}-\kappa \bar{v}+\bar{\lambda} \rho-3 \gamma \sigma+\bar{\gamma} \sigma+\mu \sigma-\bar{\alpha} \tau+\beta \tau+\tau \\
\bar{\delta} \beta-\delta \alpha & =-\Lambda-\Phi_{11}+\Psi_{2}-\alpha \bar{\alpha}+2 \alpha \beta-\beta \bar{\beta}-\epsilon \mu+\epsilon \bar{\mu}-\gamma \rho-\mu \rho+\gamma \bar{\rho}+\lambda \sigma \\
\bar{\delta} \gamma-\Delta \alpha & =\Psi_{3}-\bar{\beta} \gamma-\alpha \bar{\gamma}+\beta \lambda+\alpha \bar{\mu}-\epsilon \nu-v \rho+\lambda \tau+\gamma \bar{\tau} \\
\bar{\delta} \epsilon-D \alpha & =-\Phi_{10}+2 \alpha \epsilon+\bar{\beta} \epsilon-\alpha \bar{\epsilon}+\gamma \bar{\kappa}+\kappa \lambda-\epsilon \pi-\alpha \rho-\pi \rho-\beta \bar{\sigma} \\
\bar{\delta} \kappa-D \rho & =-\Phi_{00}+3 \alpha \kappa+\bar{\beta} \kappa-\kappa \pi-\epsilon \rho-\bar{\epsilon} \rho-\rho^{2}-\sigma \bar{\sigma}+\bar{\kappa} \tau \\
\bar{\delta} \mu-\delta \lambda & =-\Phi_{21}+\Psi_{3}-\bar{\alpha} \lambda+3 \beta \lambda-\alpha \mu-\bar{\beta} \mu-\mu \pi+\bar{\mu} \pi-v \rho+v \bar{\rho} \\
\bar{\delta} \nu-\Delta \lambda & =\Psi_{4}+3 \gamma \lambda-\bar{\gamma} \lambda+\lambda \mu+\lambda \bar{\mu}-3 \alpha \nu-\bar{\beta} \nu-v \pi+\nu \bar{\tau} \\
\bar{\delta} \pi-D \lambda & =-\Phi_{20}+3 \epsilon \lambda-\bar{\epsilon} \lambda+\bar{\kappa} \nu-\alpha \pi+\bar{\beta} \pi-\pi^{2}-\lambda \rho-\mu \bar{\sigma} \\
\bar{\delta} \sigma-\delta \rho & =-\Phi_{01}+\Psi_{1}-\kappa \mu+\kappa \bar{\mu}-\bar{\alpha} \rho-\beta \rho+3 \alpha \sigma-\bar{\beta} \sigma-\rho \tau-\bar{\rho} \tau \\
\bar{\delta} \tau-\Delta \rho & =2 \Lambda+\Psi_{2}-\kappa \nu-\gamma \rho-\bar{\gamma} \rho+\bar{\mu} \rho+\lambda \sigma+\alpha \tau-\bar{\beta} \tau+\tau \bar{\tau}
\end{aligned}
$$


Observe that the above are the full NP Ricci identities. The highlighted terms are those that vanish either because of the gauge or vacuum conditions. Further simplification following from Stewart's gauge (Lemma 1) are $\rho=\bar{\rho}, \mu=\bar{\mu}$ and $\pi=\alpha+\bar{\beta}$. Note furthermore that the three Eqs. (30j), (30n), (30q), there are constraints on the 2-spheres $\mathcal{S}_{u, v}$.

\section{The Bianchi identities}

Finally, the (second) Bianchi identities take the form:

$$
\begin{aligned}
& \bar{\delta} \Psi_{0}-D \Psi_{1}+D \Phi_{01}-\delta \Phi_{00}=(4 \alpha-\pi) \Psi_{0}-2(2 \rho+\epsilon) \Psi_{1} \\
& \quad+3 \kappa \Psi_{2}+(\bar{\tau}-2 \bar{\alpha}-2 \beta) \Phi_{00} \\
& \quad+2(\epsilon+\bar{\rho}) \Phi_{01}+2 \sigma \Phi_{10}-2 \kappa \Phi_{11}-\bar{\kappa} \Phi_{02} \\
& \Delta \Psi_{0}-\delta \Psi_{1}+D \Phi_{02}-\delta \Phi_{01}=(4 \gamma-\mu) \Psi_{0}-2(2 \tau+\beta) \Psi_{1}+3 \sigma \Psi_{2} \\
& \quad-\bar{\lambda} \Phi_{00}+2(\bar{\pi}-\beta) \Phi_{01}+2 \sigma \Phi_{11}+(\bar{\rho}+2 \epsilon-2 \bar{\epsilon}) \Phi_{02}-2 \kappa \Phi_{12} \\
& \bar{\delta} \Psi_{3}-D \Psi_{4}+\bar{\delta} \Phi_{21}-\Delta \Phi_{20}=(4 \epsilon-\rho) \Psi_{4}-2(2 \pi+\alpha) \Psi_{3}+3 \lambda \Psi_{2}+2 \lambda \Phi_{11} \\
& \quad-2 \nu \Phi_{10}-\bar{\sigma} \Phi_{22}+(2 \gamma-2 \bar{\gamma}+\bar{\mu}) \Phi_{20}+2(\bar{\tau}-\alpha) \Phi_{21} \\
& \Delta \Psi_{3}-\delta \Psi_{4}+\bar{\delta} \Phi_{22}-\Delta \Phi_{21}=(4 \beta-\tau) \Psi_{4}-2(2 \mu+\gamma) \Psi_{3}+3 \nu \Psi_{2}+2 \lambda \Phi_{12} \\
& \quad-2 \nu \Phi_{11}-\bar{v} \Phi_{20}+(\bar{\tau}-2 \bar{\beta}-2 \alpha) \Phi_{22}+2(\gamma+\bar{\mu}) \Phi_{21}, \\
& D \Psi_{2}-\bar{\delta} \Psi_{1}+\Delta \Phi_{00}-\bar{\delta} \Phi_{01}+2 D \Lambda=-\lambda \Psi_{0}+2(\pi-\alpha) \Psi_{1}+3 \rho \Psi_{2}-2 \kappa \Psi_{3} \\
& \quad-2 \tau \Phi_{10}+2 \rho \Phi_{11}+\bar{\sigma} \Phi_{02}+(2 \gamma+2 \bar{\gamma}-\bar{\mu}) \Phi_{00}-2(\bar{\tau}+\alpha) \Phi_{01} \\
& \Delta \Psi_{2}-\delta \Psi_{3}+D \Phi_{22}-\delta \Phi_{21}+2 \Delta \Lambda=\sigma \Psi_{4}+2(\beta-\tau) \Psi_{3}-3 \mu \Psi_{2}+2 \nu \Psi_{1} \\
& \quad+2 \pi \Phi_{12}-2 \mu \Phi_{11}-\bar{\lambda} \Phi_{20}+(\bar{\rho}-2 \epsilon-2 \bar{\epsilon}) \Phi_{22}+2(\bar{\pi}+\beta) \Phi_{21} \\
& \quad D \Psi_{3}-\bar{\delta} \Psi_{2}-D \Phi_{21}+\delta \Phi_{20}-2 \bar{\delta} \Lambda=-\kappa \Psi_{4}+2(\rho-\epsilon) \Psi_{3}+3 \pi \Psi_{2} \\
& \quad-2 \lambda \Psi_{1}-2 \pi \Phi_{11}+2 \mu \Phi_{10}+\bar{\kappa} \Phi_{22}+(2 \bar{\alpha}-2 \beta-\bar{\pi}) \Phi_{20}-2(\bar{\rho}-\epsilon) \Phi_{21}
\end{aligned}
$$$$
\Delta \Psi_{1}-\delta \Psi_{2}-\Delta \Phi_{01}+\bar{\delta} \Phi_{02}-2 \delta \Lambda=\nu \Psi_{0}+2(\gamma-\mu) \Psi_{1}-3 \tau \Psi_{2}+2 \sigma \Psi_{3}
$$$$
+2 \tau \Phi_{11}-2 \rho \Phi_{12}-\bar{v} \Phi_{00}+(\bar{\tau}-2 \bar{\beta}+2 \alpha) \Phi_{02}+2(\bar{\mu}-\gamma) \Phi_{01},
$$$$
D \Phi_{11}-\delta \Phi_{10}-\bar{\delta} \Phi_{01}+\Delta \Phi_{00}+3 D \Lambda=(2 \gamma-\mu+2 \bar{\gamma}-\bar{\mu}) \Phi_{00}
$$$$
+(\pi-2 \alpha-2 \bar{\tau}) \Phi_{01}+\bar{\sigma} \Phi_{02}+\sigma \Phi_{20}+(\bar{\pi}-2 \bar{\alpha}-2 \tau) \Phi_{10}
$$$$
+2(\rho+\bar{\rho}) \Phi_{11}-\bar{\kappa} \Phi_{12}-\kappa \Phi_{21},
$$$$
D \Phi_{12}-\delta \Phi_{11}-\bar{\delta} \Phi_{02}+\Delta \Phi_{01}+3 \delta \Lambda=(-2 \alpha+2 \bar{\beta}+\pi-\bar{\tau}) \Phi_{02}
$$$$
+(\bar{\rho}+2 \rho-2 \bar{\epsilon}) \Phi_{12}+\bar{v} \Phi_{00}-\bar{\lambda} \Phi_{10}+2(\bar{\pi}-\tau) \Phi_{11}
$$

$$
\begin{aligned}
& +(2 \gamma-2 \bar{\mu}-\mu) \Phi_{01}+\sigma \Phi_{21}-\kappa \Phi_{22}, \\
& D \Phi_{22}-\delta \Phi_{21}-\bar{\delta} \Phi_{12}+\Delta \Phi_{11}+3 \Delta \Lambda=(\rho+\bar{\rho} \\
& \quad-2 \epsilon-2 \bar{\epsilon}) \Phi_{22}+(2 \bar{\beta}+2 \pi-\bar{\tau}) \Phi_{12}+v \Phi_{01}+\bar{v} \Phi_{10}+(2 \beta+2 \bar{\tau}-\tau) \Phi_{21} \\
& \quad-2(\mu+\bar{\mu}) \Phi_{11}-\bar{\lambda} \Phi_{20}-\lambda \Phi_{02} .
\end{aligned}
$$


As in the case of the Ricci identities we have highlighted the vanishing terms. Note that the last three equations make no contribution to our analysis as they are satisifed identically.

\section{B: Inequalities}

In this appendix, as a quick reference, we list the key inequalities which are used routinely in our analysis. These inequalities are standard and proofs can be found, e.g. in [29].

Cauchy-Schwarz inequality If $u_{1}, \ldots, u_{n} \in \mathbb{C}$ and $v_{1}, \ldots, v_{n} \in \mathbb{C}$, we have

$$
\left|u_{1} v_{1}+\cdots+u_{n} v_{n}\right|^{2} \leq\left(\left|u_{1}\right|^{2}+\cdots+\left|u_{n}\right|^{2}\right)\left(\left|v_{1}\right|^{2}+\cdots+\left|v_{n}\right|^{2}\right) .
$$

Grönwall's inequality If $\beta(t)$ is a non-negative continuous function and $u(t)$ satisfies

$$
u(t) \leq \alpha(t)+\int_{a}^{t} \beta(s) u(s) \mathrm{d} s, \quad \forall t \in[a, b],
$$

then

$$
u(t) \leq \alpha(t)+\int_{a}^{t} \alpha(s) \beta(s) \exp \left(\int_{s}^{t} \beta(r) \mathrm{d} r\right) \mathrm{d} s, \quad t \in[a, b] .
$$

In addition, if the function $\alpha$ is non-decreasing, then

$$
u(t) \leq \alpha(t) \exp \left(\int_{a}^{t} \beta(s) \mathrm{d} s\right), t \in[a, b] .
$$

Moreover, if $\beta \equiv C$ where $C$ is a positive constant, then

$$
u(t) \leq C(b-a) \alpha(t)
$$

Young's inequality If $a$ and $b$ are non negative real numbers and $p$ and $q$ are positive real numbers such that $1 / p+1 / q=1$, then

$$
a b \leq \frac{a^{p}}{p}+\frac{b^{q}}{q} .
$$

The equality holds if and only if $a^{p}=b^{q}$. Moreover, if $a$ and $b$ are non negative real numbers and $p \geq 1$, then

$$
a^{p}+b^{p} \leq(a+b)^{p}
$$

Finally, if $f(x)$ is non-negative continuous function and $p \geq 1$, then

$$
\int_{K} f^{p} \leq\left(\int_{K} f\right)^{p}
$$


where $K$ is a compact set.

Generalised Hölder's inequality Let $K$ be a measurable space. Assume $f \in L^{p}(K)$ and $g \in L^{q}(K)$ with $1 \leq p, q \leq \infty$ and $1 / r=1 / p+1 / q \leq 1$, then

$$
\|f g\|_{L^{r}(K)} \leq\|f\|_{L^{p}(K)}\|g\|_{L^{q}(K)}
$$

Gagliardo-Nirenberg-Sobolev inequality Let $U$ be a bounded, open subset of $\mathbb{R}^{n}$, and assume $\partial U$ is $C^{1}$. Let $1 \leq p<n$, and suppose that $u \in W^{1, p}(U)$. Then $u \in L^{p *}(U)$, with the estimate,

$$
\|u\|_{L^{p *}(U)} \leq C\|u\|_{W^{1, p}(U)}
$$

the constant $C$ depending only on $p, n$ and $U$ and $1 / p+1 / p^{*}=1 / n$.

\section{C: Angular derivatives of a scalar function}

In our analysis we make repeated use of properties of the angular derivatives of a scalar field over the 2-spheres $\mathcal{S}_{u, v}$ of constant $u, v$. In the following let $f: \mathcal{S}_{u, v} \rightarrow \mathbb{C}$ denote a sufficiently smooth complex scalar field.

\section{Definitions and basic inequalities}

In terms of the NP vectors $m^{a}$ and $\bar{m}^{a}$ one has that

$$
\mathbb{\nabla}_{a} f=-m_{a} \bar{m}^{b} \not_{b} f-\bar{m}_{a} m^{b} \not_{b} f=-m_{a} \bar{\delta} f-\bar{m}_{a} \delta f .
$$

Moreover, we have that

$$
|\not \nabla|^{2} \equiv-\sigma^{a b} \bar{\nabla}_{a} f \not_{b} f=\bar{\delta} f \delta \bar{f}+\bar{\delta} \bar{f} \delta f .
$$

A direct computation shows that,

$$
\begin{aligned}
\|\not \nabla f\|_{L^{p}\left(\mathcal{S}_{u, v}\right)} & =\left(\int_{\mathcal{S}_{u, v}}|\bar{\delta} f \delta \bar{f}+\bar{\delta} \bar{f} \delta f|^{p / 2}\right)^{1 / p}=\left\||\delta f|^{2}+|\bar{\delta} f|^{2}\right\|_{L^{p / 2}\left(\mathcal{S}_{u, v}\right)}^{1 / 2} \\
& \leq\left(\left\||\delta f|^{2}\right\|_{L^{p / 2}\left(\mathcal{S}_{u, v}\right)}+\left\|\left.\bar{\delta} f\right|^{2}\right\|_{L^{p / 2}\left(\mathcal{S}_{u, v}\right)}\right)^{1 / 2} \\
& \leq\left\||\delta f|^{2}\right\|_{L^{p / 2}\left(\mathcal{S}_{u, v}\right)}^{1 / 2}+\left\|\left.\bar{\delta} f\right|^{2}\right\|_{L^{p / 2}\left(\mathcal{S}_{u, v}\right)}^{1 / 2} \\
& =\|\delta f\|_{L^{p}\left(\mathcal{S}_{u, v}\right)}+\|\bar{\delta} f\|_{L^{p}\left(\mathcal{S}_{u, v}\right)} .
\end{aligned}
$$

Conversely, we have

$$
\|\delta f\|_{L^{p}\left(\mathcal{S}_{u, v}\right)}, \quad\|\bar{\delta} f\|_{L^{p}\left(\mathcal{S}_{u, v}\right)} \leq\left(\int_{\mathcal{S}_{u, v}}|\bar{\delta} f \delta \bar{f}+\bar{\delta} \bar{f} \delta f|^{p / 2}\right)^{1 / p} \leq\|\not \nabla f\|_{L^{p}\left(\mathcal{S}_{u, v}\right)}
$$


Thus, we can estimate $\not \nabla f$ in terms of $\delta f$ and $\bar{\delta} f$ and vice versa. This observation is used repeatedly in the main text.

\section{The Hessian}

The Hessian $\nabla_{a} \nabla_{b} f$ of the scalar function $f$ can be expanded in terms of NP objects as

$$
\begin{aligned}
\mathbb{}_{a} \nabla_{b} f= & (\bar{\delta} \bar{\delta} f+(\bar{\beta}-\alpha) \bar{\delta} f) m_{a} m_{b}+(\delta \delta f+(\beta-\bar{\alpha}) \delta f) \bar{m}_{a} \bar{m}_{b} \\
& +(\bar{\delta} \delta f+(\alpha-\bar{\beta}) \delta f) m_{a} \bar{m}_{b}+(\delta \bar{\delta} f+(\bar{\alpha}-\beta) \bar{\delta} f) \bar{m}_{a} m_{b},
\end{aligned}
$$

where we have made use of the expansion

$$
\nabla_{a} m_{b}=(\alpha-\bar{\beta}) m_{a} m_{b}+(\beta-\bar{\alpha}) \bar{m}_{a} m_{b}
$$

Defining, for convenience, the scalars

$$
\begin{array}{ll}
T_{1} \equiv \bar{\delta} \bar{\delta} f+(\bar{\beta}-\alpha) \bar{\delta} f, & T_{2} \equiv \bar{\delta} \delta f+(\alpha-\bar{\beta}) \delta f, \\
T_{3} \equiv \delta \bar{\delta} f+(\bar{\alpha}-\beta) \bar{\delta} f, & T_{4} \equiv \delta \delta f+(\beta-\bar{\alpha}) \delta f,
\end{array}
$$

one can then write

$$
\left|\nabla^{2} f\right|^{2} \equiv \sigma^{a b} \sigma^{c d} \bar{\nabla}_{a} \nabla_{c} f \nabla_{b} \nabla_{d} f=\left|T_{1}\right|^{2}+\left|T_{2}\right|^{2}+\left|T_{3}\right|^{2}+\left|T_{4}\right|^{2}
$$

Making use of the above decomposition we then have that

$$
\begin{aligned}
\left\|\nabla^{2} f\right\|_{L^{p}\left(\mathcal{S}_{u, v}\right)}= & \left(\int_{\mathcal{S}_{u, v}}\left(\left|T_{1}\right|^{2}+\left|T_{2}\right|^{2}+\left|T_{3}\right|^{2}+\left|T_{4}\right|^{2}\right)^{p / 2}\right)^{1 / p} \leq \sum_{i=1}^{4}\left\|T_{i}\right\|_{L^{p}\left(\mathcal{S}_{u, v}\right)} \\
\leq & \left\|\delta^{2} f\right\|_{L^{p}\left(\mathcal{S}_{u, v}\right)}+\left\|\bar{\delta}^{2} f\right\|_{L^{p}\left(\mathcal{S}_{u, v}\right)}+\|\delta \bar{\delta} f\|_{L^{p}\left(\mathcal{S}_{u, v}\right)}+\|\bar{\delta} \delta f\|_{L^{p}\left(\mathcal{S}_{u, v}\right)} \\
& +4 \Delta_{\Gamma}\left(\|\delta f\|_{L^{p}\left(\mathcal{S}_{u, v}\right)}+\|\bar{\delta} f\|_{L^{p}\left(\mathcal{S}_{u, v}\right)}\right)
\end{aligned}
$$

where $\Delta_{\Gamma}$ is defined as in the main text. Also, observe that $\left\|\not \nabla^{2} f\right\|_{L^{p}\left(\mathcal{S}_{u, v}\right)}$ is not smaller than any of the individual terms in the right side of the first inequality (32).

A final observation following the irreducible decomposition

$$
\nabla_{a} \nabla_{b} f=\not_{\{a} \nabla_{b\}} f+\frac{1}{2} \sigma_{a b} \not f+\not_{[a} \nabla_{b]} f
$$

of the Hessian, where the curly brackets denote the symmetric-tracefree part with respect to the metric $\sigma_{a b}$, is that

$$
\left|\nabla_{a} \nabla_{b} f\right|^{2}=\left|\nabla_{\{a} \nabla_{b\}} f\right|^{2}+\frac{1}{2}|\not \forall f|^{2}+\left|\not_{[a} \nabla_{b]} f\right|^{2}
$$


so that

$$
|\not \Delta f|^{2} \leq 2\left|\nabla_{a} \nabla_{b} f\right|^{2}
$$

\section{Third derivatives of a scalar field}

As in the main text denote by $\varpi \equiv \beta-\bar{\alpha}$ the simple independent component of the connection of the 2 -sphere $\mathcal{S}_{u, v}$. It follows from the from the structure Eq. (30j) and its complex conjugate, that the Gaussian curvature curvature

$$
K \equiv 2 \varpi \bar{\varpi}+2 \delta \bar{\varpi}+2 \bar{\delta} \varpi
$$

satisfies the relation

$$
K=\sigma \lambda+\bar{\sigma} \bar{\lambda}-\rho \mu-\bar{\rho} \bar{\mu}+\Psi_{2}+\bar{\Psi}_{2},
$$

see [27] for details.

Now, the third order covariant derivative of $f$ on $\mathcal{S}_{u, v}$ can be expanded as

$$
\begin{aligned}
\nabla_{a} \nabla_{b} \nabla_{c} f= & M_{1} m_{a} m_{b} m_{c}+M_{5} \bar{m}_{a} \bar{m}_{b} \bar{m}_{c}+M_{2} \bar{m}_{a} m_{b} m_{c}+M_{6} m_{a} \bar{m}_{b} \bar{m}_{c} \\
& +M_{3} m_{a} m_{b} \bar{m}_{c}+M_{7} \bar{m}_{a} \bar{m}_{b} m_{c}+M_{4} \bar{m}_{a} m_{b} \bar{m}_{c}+M_{8} m_{a} \bar{m}_{b} m_{c},
\end{aligned}
$$

where,

$$
\begin{aligned}
& M_{1} \equiv-\left(\bar{\delta}^{3} f+3 \bar{\varpi} \bar{\delta}^{2} f+\bar{\delta} \bar{\varpi} \bar{\delta} f+2 \bar{\varpi}^{2} \bar{\delta} f\right), \\
& M_{2} \equiv-\delta \bar{\delta}^{2} f-\bar{\varpi} \delta \bar{\delta} f+2 \varpi \bar{\delta}^{2} f-\delta \bar{\varpi} \bar{\delta} f+2 \varpi \bar{\varpi} \bar{\delta} f, \\
& M_{3} \equiv-\bar{\delta}^{2} \delta f+\bar{\varpi} \bar{\delta} \delta f+\bar{\delta} \bar{\varpi} \delta f, \\
& M_{4} \equiv-\delta \bar{\delta} \delta f+\bar{\varpi} \delta^{2} f+\delta \bar{\varpi} \delta f, \\
& M_{5} \equiv-\left(\delta^{3} f+3 \varpi \delta^{2} f+\delta \varpi \delta f+2 \varpi^{2} \delta f\right), \\
& M_{6} \equiv-\bar{\delta} \delta^{2} f-\varpi \delta \delta \delta f+2 \bar{\varpi} \delta^{2} f-\bar{\delta} \varpi \delta f+2 \varpi \bar{\varpi} \delta f, \\
& M_{7} \equiv-\delta^{2} \bar{\delta} f+\varpi \delta \bar{\delta} f+\delta \varpi \delta f, \\
& M_{8} \equiv-\bar{\delta} \delta \bar{\delta} f+\varpi \bar{\delta}^{2} f+\bar{\delta} \varpi \bar{\delta} f .
\end{aligned}
$$

It follows then that,

$$
\left|\nabla^{3} f\right|^{2}=\sum_{i=1}^{8}\left|M_{i}\right|^{2} .
$$

From the above expression one finds that

$$
\begin{aligned}
& \left\|\not \nabla^{3} f\right\|_{L^{p}\left(\mathcal{S}_{u, v}\right)} \\
& \quad \leq\left\|\delta^{3} f\right\|_{L^{p}\left(\mathcal{S}_{u, v}\right)}+\left\|\bar{\delta}^{3} f\right\|_{L^{p}\left(\mathcal{S}_{u, v}\right)}+\left\|\delta^{2} \bar{\delta} f\right\|_{L^{p}\left(\mathcal{S}_{u, v}\right)}+\left\|\delta \bar{\delta}^{2} f\right\|_{L^{p}\left(\mathcal{S}_{u, v}\right)}
\end{aligned}
$$




$$
\begin{aligned}
& +\left\|\bar{\delta}^{2} \delta f\right\|_{L^{p}\left(\mathcal{S}_{u, v}\right)}+\left\|\bar{\delta} \delta^{2} f\right\|_{L^{p}\left(\mathcal{S}_{u, v}\right)}+\|\delta \bar{\delta} \delta f\|_{L^{p}\left(\mathcal{S}_{u, v}\right)}+\|\bar{\delta} \delta \bar{\delta} f\|_{L^{p}\left(\mathcal{S}_{u, v}\right)} \\
& +3\left\|\bar{\varpi} \bar{\delta}^{2} f\right\|_{L^{p}\left(\mathcal{S}_{u, v}\right)}+\|\bar{\varpi} \delta \bar{\delta} f\|_{L^{p}\left(\mathcal{S}_{u, v}\right)}+2\left\|\varpi \bar{\delta}^{2} f\right\|_{L^{p}\left(\mathcal{S}_{u, v}\right)}+\|\bar{\varpi} \delta \delta\|_{L^{p}\left(\mathcal{S}_{u, v}\right)} \\
& +\left\|\bar{\varpi} \delta^{2} f\right\|_{L^{p}\left(\mathcal{S}_{u, v}\right)}+3\left\|\varpi \delta^{2} f\right\|_{L^{p}\left(\mathcal{S}_{u, v}\right)}+\|\varpi \bar{\delta} \delta f\|_{L^{p}\left(\mathcal{S}_{u, v}\right)}+2\left\|\bar{\varpi} \delta^{2} f\right\|_{L^{p}\left(\mathcal{S}_{u, v}\right)} \\
& +\|\varpi \delta \bar{\delta} f\|_{L^{p}\left(\mathcal{S}_{u, v}\right)}+\left\|\overline{\delta^{2}} f\right\|_{L^{p}\left(\mathcal{S}_{u, v}\right)}+\|\bar{\delta} \bar{\varnothing} \bar{\delta} f\|_{L^{p}\left(\mathcal{S}_{u, v}\right)}+\|\delta \bar{\varpi} \bar{\delta} f\|_{L^{p}\left(\mathcal{S}_{u, v}\right)} \\
& +\|\bar{\delta} \bar{\varpi} \delta f\|_{L^{p}\left(\mathcal{S}_{u, v}\right)}+\|\delta \bar{\varpi} \delta f\|_{L^{p}\left(\mathcal{S}_{u, v}\right)}+\|\delta \varpi \delta f\|_{L^{p}\left(\mathcal{S}_{u, v}\right)}+\|\bar{\delta} \varpi \delta f\|_{L^{p}\left(\mathcal{S}_{u, v}\right)} \\
& +\|\delta \varpi \bar{\delta} f\|_{L^{p}\left(\mathcal{S}_{u, v}\right)}+\|\bar{\delta} \varpi \bar{\delta} f\|_{L^{p}\left(\mathcal{S}_{u, v}\right)}+2\left\|\bar{\varpi}^{2} \bar{\delta} f\right\|_{L^{p}\left(\mathcal{S}_{u, v}\right)}+2\|\varpi \bar{\varpi} \bar{\delta} f\|_{L^{p}\left(\mathcal{S}_{u, v}\right)} \\
& +2\left\|\varpi^{2} \delta f\right\|_{L^{p}\left(\mathcal{S}_{u, v}\right)}+2\left\|\varpi \bar{\varpi}^{2} \delta f\right\|_{L^{p}\left(\mathcal{S}_{u, v}\right)} .
\end{aligned}
$$

The above expression contains four representative terms, namely $\left\|\delta^{3} f\right\|_{L^{p}\left(\mathcal{S}_{u, v}\right)}$, $\left\|\varpi \delta^{2} f\right\|_{L^{p}\left(\mathcal{S}_{u, v}\right)},\|\delta \varpi \delta f\|_{L^{p}\left(\mathcal{S}_{u, v}\right)}$ and $\left\|\varpi^{2} \delta f\right\|_{L^{p}\left(\mathcal{S}_{u, v}\right)}$ which will be used to illustrate the analysis in the main text.

\section{D: Integration Identities}

In this appendix we prove some integration identities which are routinely used in the main text.

First we observe that a direct calculation yields

$$
\begin{aligned}
\not_{\mathcal{A}} P^{\mathcal{A}} & =\frac{1}{\sqrt{\operatorname{det} \sigma}} \partial_{\mathcal{A}}\left(\sqrt{\operatorname{det} \sigma} P^{\mathcal{A}}\right)=\frac{P^{\mathcal{A}}}{\sqrt{\operatorname{det} \sigma}} \partial_{\mathcal{A}} \sqrt{\operatorname{det} \sigma}+\partial_{\mathcal{A}} P^{\mathcal{A}} \\
& =\frac{1}{2} P^{\mathcal{A}} \sigma^{\mathcal{B C}} \partial_{\mathcal{A}} \sigma_{\mathcal{B C}}+\partial_{\mathcal{A}} P^{\mathcal{A}}=-\frac{1}{2} P^{\mathcal{A}} \sigma_{\mathcal{B C}} \partial_{\mathcal{A}} \sigma^{\mathcal{B C}}+\partial_{\mathcal{A}} P^{\mathcal{A}} \\
& =P^{\mathcal{A}} P^{\mathcal{B}} \sigma_{\mathcal{B C}} \partial_{\mathcal{A}} \bar{P}^{\mathcal{C}}+P^{\mathcal{A}} \bar{P}^{\mathcal{C}} \sigma_{\mathcal{B C}} \partial_{\mathcal{A}} P^{\mathcal{B}}-\bar{m}_{\mathcal{C}} \delta P^{\mathcal{C}}-m_{\mathcal{C}} \bar{\delta} P^{\mathcal{C}}, \\
& =\sigma_{\mathcal{B C}} P^{\mathcal{B}}\left(\delta \bar{P}^{\mathcal{C}}-\bar{\delta} P^{\mathcal{C}}\right)=\varpi .
\end{aligned}
$$

In the last step we have made use of Eq. (12e). Consequently, we also have that $\nabla_{\mathcal{A}} \bar{P}^{\mathcal{A}}=\bar{\varpi}$. Making use of these results we further compute on the arbitrary sphere $\mathcal{S}$ that

$$
\begin{aligned}
\int_{\mathcal{S}}|\delta f|^{2} & =\int_{\mathcal{S}} \delta f \bar{\delta} \bar{f} \sqrt{\operatorname{det} \sigma} \mathrm{d}^{2} x=\int_{\mathcal{S}} \delta f \bar{P}^{\mathcal{A}} \partial_{\mathcal{A}} \bar{f} \sqrt{\operatorname{det} \sigma} \mathrm{d}^{2} x, \\
& =-\int_{\mathcal{S}} \bar{f} \bar{P}_{\mathcal{A}}\left(\delta f \bar{P}^{\mathcal{A}}\right)=-\int_{\mathcal{S}} \bar{f} \bar{\delta} \delta f-\int_{\mathcal{S}} \bar{f} \nabla_{\mathcal{A}} \bar{P}^{\mathcal{A}} \delta f .
\end{aligned}
$$

On the one hand, the first integral in the last equality can be further expanded as

$$
\begin{aligned}
\int_{\mathcal{S}} \bar{f} \bar{\delta} \delta f & =\int_{\mathcal{S}} \bar{f} \delta \bar{\delta} f-\int_{\mathcal{S}} \bar{f} \bar{\varpi} \delta f+\int_{\mathcal{S}} \bar{f} \varpi \bar{\delta} f \\
& =\int_{\mathcal{S}} \bar{f} P^{\mathcal{A}} \partial_{\mathcal{A}} \bar{\delta} f-\int_{\mathcal{S}} \bar{f} \bar{\varpi} P^{\mathcal{A}} \partial_{\mathcal{A}} f+\int_{\mathcal{S}} \bar{f} \varpi \bar{\delta} f
\end{aligned}
$$




$$
\begin{aligned}
& =-\int_{\mathcal{S}} \bar{\delta} f \nabla_{\mathcal{A}}\left(\bar{f} P^{\mathcal{A}}\right)+\int_{\mathcal{S}} f \nabla_{\mathcal{A}}\left(\bar{f} \bar{\varpi} P^{\mathcal{A}}\right)+\int_{\mathcal{S}} \bar{f} \varpi \bar{\delta} f \\
& =-\int_{\mathcal{S}}|\bar{\delta} f|^{2}+\int_{\mathcal{S}}|f|^{2}|\varpi|^{2}+\int_{\mathcal{S}}|f|^{2} \delta \bar{\varpi}+\int_{\mathcal{S}} f \bar{\varpi} \delta \bar{f} .
\end{aligned}
$$

On the other hand, the second integral can be expanded as

$$
\begin{aligned}
\int_{\mathcal{S}} \bar{f} \nabla_{\mathcal{A}} \bar{P}^{\mathcal{A}} \delta f & =\int_{\mathcal{S}} \bar{f} \bar{\varpi} \delta f=\int_{\mathcal{S}} \bar{f} \bar{\varpi} P^{\mathcal{A}} \nabla_{\mathcal{A}} f=-\int_{\mathcal{S}} f \nabla_{\mathcal{A}}\left(\bar{f} \bar{\varpi} P^{\mathcal{A}}\right) \\
& =-\int_{\mathcal{S}} f \bar{\varpi} P^{\mathcal{A}} \nabla_{\mathcal{A}} \bar{f}-\int_{\mathcal{S}}|f|^{2} P^{\mathcal{A}} \nabla_{\mathcal{A}} \bar{\varpi}-\int_{\mathcal{S}}|f|^{2} \bar{\varpi} \nabla_{\mathcal{A}} P^{\mathcal{A}} \\
& =-\int_{\mathcal{S}} f \bar{\varpi} \delta \bar{f}-\int_{\mathcal{S}}|f|^{2} \delta \bar{\varpi}-\int_{\mathcal{S}}|f|^{2}|\varpi|^{2} .
\end{aligned}
$$

Combining these last expressions one finds that

$$
\int_{\mathcal{S}}|\delta f|^{2}=\int_{\mathcal{S}}|\bar{\delta} f|^{2}
$$

In other words we have found that

$$
\|\delta f\|_{L^{2}\left(\mathcal{S}_{u, v}\right)}=\|\bar{\delta} f\|_{L^{2}\left(\mathcal{S}_{u, v}\right)} .
$$

\section{E: Details in Propositions 8 and 9}

In this appendix we provide further details regarding the lengthy computations arising in the analysis of Propositions 8 and 9.

\section{Estimates on the $L^{4}$-norm of connection coefficients}

In the following we consider, for conciseness, the NP spin connection coefficient $\lambda$. Making use of Proposition 2 to estimate $\|\lambda\|_{L^{4}(S)}$ one finds that

$$
\|\not \nabla \lambda\|_{L^{4}\left(\mathcal{S}_{u, v}\right)} \leq 2\left(\|\nabla \lambda\|_{L^{4}\left(\mathcal{S}_{0, v}\right)}+C\left(\Delta_{e_{\star}}, \Delta_{\Gamma_{\star}}\right) \int_{0}^{u}\left(\int_{\mathcal{S}_{u^{\prime}, v}} \Delta\langle\not \nabla \lambda, \nabla \nabla\rangle_{\sigma}^{2}\right)^{1 / 4} \mathrm{~d} u^{\prime}\right) .
$$

One can then estimate

$$
\begin{aligned}
\int_{\mathcal{S}_{u^{\prime}, v}}\left|\Delta\langle\not \nabla \lambda, \not \nabla \lambda\rangle_{\sigma}^{2}\right| & =\int_{\mathcal{S}_{u^{\prime}, v}}|\not \nabla \lambda|^{2}|\Delta(\bar{\delta} \lambda \delta \bar{\lambda}+\bar{\delta} \bar{\lambda} \delta \lambda)| \\
& =\int_{\mathcal{S}_{u^{\prime}, v}}|\not \nabla \lambda|^{2}|(\Delta \delta \lambda) \bar{\delta} \bar{\lambda}+\delta \lambda \Delta \bar{\delta} \bar{\lambda}+\delta \bar{\lambda} \Delta \bar{\delta} \lambda+\bar{\delta} \lambda \Delta \delta \bar{\lambda}|
\end{aligned}
$$




$$
\begin{aligned}
& \leq \int_{S_{u^{\prime}, v}}|\nabla \lambda|^{2} \sqrt{2|\delta \lambda|^{2}+2|\bar{\delta} \lambda|^{2}} \sqrt{2|\Delta \delta \lambda|^{2}+2|\Delta \bar{\delta} \lambda|^{2}} \\
& \leq 2 \int_{\mathcal{S}_{u^{\prime}, v}}|\not \nabla \lambda|^{3}(|\Delta \delta \lambda|+|\Delta \bar{\delta} \lambda|),
\end{aligned}
$$

where we have made use of the Cauchy-Schwarz inequality in the first inequality. Now, making use of the expressions for $\Delta \delta \lambda$ and $\Delta \bar{\delta} \lambda$ one further finds that,

$$
\begin{aligned}
& \int_{\mathcal{S}_{u^{\prime}, v}}\left|\Delta\langle\not \nabla \lambda, \not \nabla \lambda\rangle_{\sigma}^{2}\right| \leq 2 \int_{\mathcal{S}_{u^{\prime}, v}}|\not \nabla \lambda|^{3}\left(|\Gamma|^{3}+|\Gamma|\left|\Psi_{4}\right|+\left|\Gamma^{\prime}\right||\not \nabla \lambda|+4|\lambda||\not \nabla \mu|+\left|\not \nabla \Psi_{4}\right|\right) \\
& \leq C\left(I, \Delta_{e_{\star}}, \Delta_{\Gamma_{\star}}, \Delta \Psi(\mathcal{S})\right)\left(\int_{\mathcal{S}_{u^{\prime}, v}}|\nabla \lambda|^{3}+|| \Psi_{4} \|_{L^{\infty}\left(\mathcal{S}_{u^{\prime}, v}\right)} \int_{\mathcal{S}_{u^{\prime}, v}}|\nabla \lambda|^{3}\right) \\
& +C\left(\Delta_{\Gamma_{\star}}\right) \int_{\mathcal{S}_{u^{\prime}, v}}|\not \nabla \lambda|^{4}+C\left(\Delta_{\Gamma_{\star}}\right) \int_{\mathcal{S}_{u^{\prime}, v}}|\not \nabla \lambda|^{3}|\not \nabla \mu|+2|| \not \nabla \Psi_{4} \|_{L^{\infty}\left(\mathcal{S}_{u^{\prime}, v}\right)} \int_{\mathcal{S}_{u^{\prime}, v}}|\not \nabla \lambda|^{3} \\
& \leq C\left(I, \Delta_{e_{\star}}, \Delta_{\Gamma_{\star}}, \Delta_{\Psi}(\mathcal{S})\right) \operatorname{Area}\left(\mathcal{S}_{u^{\prime}, v}\right)^{1 / 4}\|\not \nabla \lambda\|_{L^{4}\left(\mathcal{S}_{u^{\prime}, v}\right)}^{3}\left(1+\left(\sum_{i=0}^{2}\left\|\nabla^{i} \Psi_{4}\right\|_{L^{2}\left(\mathcal{S}_{u^{\prime}, v}\right.}\right)\right) \\
& +C\left(\Delta_{\Gamma_{\star}}\right)\|\not \nabla \lambda\|_{L^{4}\left(\mathcal{S}_{u^{\prime}, v}\right)}^{3}\|\not \nabla \mu\|_{L^{4}\left(\mathcal{S}_{u^{\prime}, v}\right)}+C\left(\Delta_{\Gamma_{\star}}\right)\|\not \nabla \lambda\|_{L^{4}\left(\mathcal{S}_{u^{\prime}, v}\right)}^{4} \\
& +C\left(\Delta_{\Gamma_{\star}}\right) \operatorname{Area}\left(\mathcal{S}_{u^{\prime}, v}\right)^{1 / 4}\|\not \nabla \lambda\|_{L^{4}\left(\mathcal{S}_{u^{\prime}, v}\right)}^{3}\left(\sum_{i=1}^{3}\left\|\nabla^{i} \Psi_{4}\right\|_{L^{2}\left(\mathcal{S}_{u^{\prime}, v}\right)}\right),
\end{aligned}
$$

where in the previous chain of inequalities we have made use of Hölder's inequality and the Sobolev's embedding. Moreover, here $\Gamma$ represents a linear combination of the NP spin connection coefficients $\tau, \alpha, \beta, \mu, \lambda$ whereas $\Gamma^{\prime}$ contains no $\tau$ term, which allows the use of sharper estimates. Both $\Gamma$ and $\Gamma^{\prime}$ are controlled in $L^{\infty}\left(\mathcal{S}_{u^{\prime}, v}\right)$ as a result of Proposition 7.

Making use of the latter estimate and of the bootstrap assumption in Proposition 8, one readily obtains that

$\|\not \nabla \lambda\|_{L^{4}\left(\mathcal{S}_{u, v}\right)} \leq 2 \Delta_{\Gamma_{\star}}+C\left(I, \Delta_{e_{\star}}, \Delta_{\Gamma_{\star}}, \Delta_{\Psi}(\mathcal{S})\right) \varepsilon+C\left(I, \Delta_{e_{\star}}, \Delta_{\Gamma_{\star}}, \Delta_{\Psi}(\mathcal{S})\right) \Delta_{\Psi} \varepsilon^{7 / 8}$

where it has been used that

$$
\begin{aligned}
\int_{0}^{u}\left(\int_{\mathcal{S}_{u^{\prime}, v}}\left|\Psi_{4}\right|^{2}\right)^{1 / 8} \mathrm{~d} u^{\prime} & \leq\left(\int_{0}^{u} \int_{\mathcal{S}_{u^{\prime}, v}}\left|\Psi_{4}\right|^{2} \mathrm{~d} u^{\prime}\right)^{1 / 8}\left(\int_{0}^{u} 1 \mathrm{~d} u^{\prime}\right)^{7 / 8} \\
& \leq \varepsilon^{7 / 8}|| \Psi_{4} \|_{L^{2}\left(\mathcal{N}_{v}(0, u)\right)}^{1 / 4}
\end{aligned}
$$

Thus, we can choose a suitable $\varepsilon>0$ such that $\|\not \lambda\|_{L^{4}\left(\mathcal{S}_{u, v}\right)} \leq 3 \Delta_{\Gamma_{\star}}$. This improves the starting bootstrap assumption. 


\section{Estimates on $\left\|\nabla^{2} \lambda\right\|_{L^{2}\left(\mathcal{S}_{u, v}\right)}$}

In this case we start from

$$
\begin{aligned}
\int_{\mathcal{S}_{u, v}}\left|\Delta\left\langle\not \nabla^{2} \lambda, \not \nabla^{2} \lambda\right\rangle_{\sigma}\right| & =\int_{\mathcal{S}_{u, v}} 2\left|\Delta\left(T_{1} \bar{T}_{1}+T_{2} \bar{T}_{2}+T_{3} \bar{T}_{3}+T_{4} \bar{T}_{4}\right)\right| \\
& \leq 2 \sqrt{2} \int_{\mathcal{S}_{u, v}}\left|\nabla^{2} \lambda\right|\left(\left|\Delta T_{1}\right|+\left|\Delta T_{2}\right|+\left|\Delta T_{3}\right|+\left|\Delta T_{4}\right|\right) .
\end{aligned}
$$

we can then further expand to obtain (in schematic notation for simplicity) that

$$
\begin{aligned}
& \int_{\mathcal{S}_{u^{\prime}, v}} \mid \Delta\left\langle\nabla^{2} \lambda,\left.\not \nabla^{2} \lambda\right|_{\sigma}\left|\leq 2 \sqrt{2} \int_{\mathcal{S}_{u^{\prime}, v}}\right| \not \nabla^{2} \lambda\right|\left(\left|\Gamma^{\prime}\right|\left|\nabla^{2} \lambda\right|+\left|\Gamma^{\prime}\right|\left|\not^{2} \Gamma\right|+\left|\not \nabla^{2} \Psi_{4}\right|\right. \\
& \left.+|\not \nabla \Gamma||\not \nabla \Gamma|+\left|\Gamma^{2}\right||\not \nabla \Gamma|+\left|\Psi_{4}\right||\not \nabla \Gamma|+\left|\Psi_{3}\right||\not \nabla \lambda|+|\Gamma|\left|\not \nabla \Psi_{4}\right|+\left|\Psi_{4}\right|\left|\Gamma^{2}\right|+\left|\Gamma^{4}\right|\right) \\
& \leq C\left(\Delta_{\Gamma_{\star}}\right) \int_{\mathcal{S}_{u^{\prime}, v}}\left|\not \nabla^{2} \lambda\right|^{2}+C\left(\Delta_{\Gamma_{\star}}\right) \int_{\mathcal{S}_{u^{\prime}, v}}\left|\nabla^{2} \lambda\right|\left|\nabla^{2} \Gamma\right|+\int_{\mathcal{S}_{u^{\prime}, v}}\left|\not \nabla^{2} \lambda\right|\left|\nabla^{2} \Psi_{4}\right| \\
& +\int_{\mathcal{S}_{u^{\prime}, v}}\left|\not \nabla^{2} \lambda\right||\not| \Gamma|| \not|\Gamma|+C\left(I, \Delta_{e_{\star}}, \Delta_{\Gamma_{\star}}, \Delta_{\Psi}(\mathcal{S})\right) \int_{\mathcal{S}_{u^{\prime}, v}}\left|\nabla^{2} \lambda\right||\not| \Gamma \mid \\
& +C\left(\Delta_{\Psi}(\mathcal{S})\right) \int_{\mathcal{S}_{u^{\prime}, v}}\left|\nabla^{2} \lambda\right||\not \nabla \Gamma| \\
& +C\left(I, \Delta_{e_{\star}}, \Delta_{\Gamma_{\star}}, \Delta_{\Psi}(\mathcal{S})\right) \int_{\mathcal{S}_{u^{\prime}, v}}\left|\not \nabla^{2} \lambda\right|\left|\not \nabla \Psi_{4}\right|+C\left(I, \Delta_{e_{\star}}, \Delta_{\Gamma_{\star}}, \Delta_{\Psi}(\mathcal{S})\right) \int_{\mathcal{S}_{u^{\prime}, v}}\left|\not{ }^{2} \lambda\right| \\
& +\int_{\mathcal{S}_{u^{\prime}, v}}\left|\not \nabla^{2} \lambda\right||\not \nabla \Gamma|\left|\Psi_{4}\right|+C\left(I, \Delta_{e_{\star}}, \Delta_{\Gamma_{\star}}, \Delta_{\Psi}(\mathcal{S})\right) \int_{\mathcal{S}_{u^{\prime}, v}}\left|\not \nabla^{2} \lambda\right|\left|\Psi_{4}\right| \\
& \leq C\left(\Delta_{\Gamma_{\star}}\right)\left\|\not \nabla^{2} \lambda\right\|_{L^{2}\left(\mathcal{S}_{u^{\prime}, v}\right)}^{2}+C\left(\Delta_{\Gamma_{\star}}\right)\left\|\nabla^{2} \lambda\right\|_{L^{2}\left(\mathcal{S}_{u^{\prime}, v}\right)}\left\|\not \nabla^{2} \Gamma\right\|_{L^{2}\left(\mathcal{S}_{u^{\prime}, v}\right)} \\
& +\left\|\not \nabla^{2} \lambda\right\|_{L^{2}\left(\mathcal{S}_{u^{\prime}, v}\right)}\left\|\not \nabla^{2} \Psi_{4}\right\|_{L^{2}\left(\mathcal{S}_{u^{\prime}, v}\right)}+\left\|\not \nabla^{2} \lambda\right\|_{L^{2}\left(\mathcal{S}_{u^{\prime}, v}\right)}\|\not \nabla \Gamma\|_{L^{4}\left(\mathcal{S}_{u^{\prime}, v}\right)}^{2} \\
& \left.+C\left(I, \Delta_{e_{\star}}, \Delta_{\Gamma_{\star}}, \Delta_{\Psi}(\mathcal{S})\right)\left\|\nabla^{2} \lambda\right\|_{L^{2}\left(\mathcal{S}_{u^{\prime}, v}\right.}\right)\|\not \nabla \Gamma\|_{L^{4}\left(\mathcal{S}_{u^{\prime}, v}\right)} \\
& +C\left(\Delta_{e_{\star}}, \Delta_{\Gamma_{\star}}\right)\left\|\Psi_{4}\right\|_{L^{\infty}\left(\mathcal{S}_{u^{\prime}, v}\right)}\left\|\nabla^{2} \lambda\right\|_{L^{2}\left(\mathcal{S}_{u^{\prime}, v}\right)} \\
& +C\left(I, \Delta_{e_{\star}}, \Delta_{\Gamma_{\star}}, \Delta_{\Psi}(\mathcal{S})\right)\left\|\not \nabla^{2} \lambda\right\|_{L^{2}\left(\mathcal{S}_{u^{\prime}, v}\right)}\left\|\not \nabla \Psi_{4}\right\|_{L^{2}\left(\mathcal{S}_{u^{\prime}, v}\right)} \\
& +C\left(I, \Delta_{e_{\star}}, \Delta_{\Gamma_{\star}}, \Delta_{\Psi}(\mathcal{S})\right)\left\|\not \nabla^{2} \lambda\right\|_{L^{2}\left(\mathcal{S}_{u^{\prime}, v}\right)} \\
& +C\left(I, \Delta_{e_{\star}}, \Delta_{\Gamma_{\star}}, \Delta_{\Psi}(\mathcal{S})\right)\left\|\not \nabla^{2} \lambda\right\|_{L^{2}\left(\mathcal{S}_{u^{\prime}, v}\right)}\|\not \nabla \Gamma\|_{L^{4}\left(\mathcal{S}_{u^{\prime}, v}\right)}\left\|\Psi_{4}\right\|_{L^{\infty}\left(\mathcal{S}_{u^{\prime}, v}\right)} \\
& \leq C\left(I, \Delta_{e_{\star}}, \Delta_{\Gamma_{\star}}, \Delta_{\Psi}(\mathcal{S})\right)\left(1+\left\|\Psi_{4}\right\|_{L^{2}\left(\mathcal{S}_{u^{\prime}, v}\right)}+\left\|\not \nabla \Psi_{4}\right\|_{L^{2}\left(\mathcal{S}_{u^{\prime}, v}\right)}+\left\|\not \nabla^{2} \Psi_{4}\right\|_{L^{2}\left(\mathcal{S}_{u^{\prime}, v}\right)}\right) \text {. }
\end{aligned}
$$

In the previous chain of inequalities we have made repeated use of our bootstrap assumption, the results in Proposition 4 and of Hölder's inequality. Finally, combining with the short direction estimate in Proposition 2 we conclude that

$$
\left\|\nabla^{2} \lambda\right\|_{L^{2}\left(\mathcal{S}_{u, v}\right)} \leq 2 \Delta_{\Gamma_{\star}}+C\left(I, \Delta_{e_{\star}}, \Delta_{\Gamma_{\star}}, \Delta_{\Psi}(\mathcal{S})\right) \varepsilon+C\left(I, \Delta_{e_{\star}}, \Delta_{\Gamma_{\star}}, \Delta_{\Psi}(\mathcal{S})\right) \Delta_{\Psi} \varepsilon^{3 / 4}
$$


The factor $\varepsilon^{3 / 4}$ results from the transferring of the 2-sphere estimate of $\Psi_{4}$ to the light cone.

\section{References}

1. Bondi, H., van der Burg, M.G.J., Metzner, A.W.K.: Gravitational waves in general relativity VII. Waves from axi-symmetric isolated systems. Proc. R. Soc. Lond. A 269, 21 (1962)

2. Sachs, R.K.: Gravitational waves in general relativity VIII. Waves in asymptotically flat space-time. Proc. R. Soc. Lond. A 270, 103 (1962)

3. Sachs, R.K.: On the characteristic initial value problem in gravitational theory. J. Math. Phys. 3, 908 (1962)

4. Newman, E.T., Penrose, R.: An approach to gravitational radiation by a method of spin coefficients. J. Math. Phys. 3, 566 (1962)

5. Penrose, R.: Zero rest-mass fields including gravitation: asymptotic behaviour. Proc. R. Soc. Lond. A 284, 159 (1965)

6. Müller zu Hagen, H., Seifert, H.-J.: On characteristic initial-value and mixed problems. Gen. Relativ. Gravit 8, 259 (1977)

7. Friedrich, H.: On the regular and the asymptotic characteristic initial value problem for Einstein's vacuum field equations. Proc. R. Soc. Lond. A 375, 169 (1981)

8. Friedrich, H.: The asymptotic characteristic initial value problem for Einstein's vacuum field equations as an initial value problem for a first-order quasilinear symmetric hyperbolic system. Proc. R. Soc. Lond. A 378, 401 (1981)

9. Friedrich, H.: On the existence of analytic null asymptotically flat solutions of Einstein's vacuum field equations. Proc. R. Soc. Lond. A 381, 361 (1982)

10. Stewart, J.M., Friedrich, H.: Numerical relativity. The characteristic initial value problem. Proc. R. Soc. Lond. A 384, 427 (1982)

11. Isaacson, R.A., Welling, J.S., Winicour, J.: Null cone computation for gravitational radiation. J. Math. Phys. 24, 1824 (1983)

12. Friedrich, H., Stewart, J.: Characteristic initial data and wavefront singularities in general relativity. Proc. R. Soc. Lond. A 385, 345 (1983)

13. Rendall, A.D.: Reduction of the characteristic initial value problem to the cauchy problem and its application to the einstein equations. Proc. R. Soc. Lond. A 427, 221 (1990)

14. Fourès-Bruhat, Y.: Théorème d'existence pour certains systèmes d'équations aux derivées partielles non linéaires. Acta Math. 88, 141 (1952)

15. Christodoulou, D., Klainerman, S.: Asymptotic properties of linear field equations in minkowski spacetime. Commun. Pure Appl. Math. 43, 137 (1990)

16. Christodoulou, D., Klainerman, S.: The Global Nonlinear Stability of the Minkowski Space. Princeton University Press, Princeton (1993)

17. Christodoulou, D.: The Formation of Black Holes in General Relativity. European Mathematical Society Publishing House, Zurich (2009). Number v. 4 in EMS monographs in mathematics

18. Luk, J.: On the local existence for the characteristic initial value problem in general relativity. Int. Math. Res. Not. 20, 4625 (2012)

19. Li, J., Zhu, X.-P.: On the local extension of the future null infinity. J. Diff. Geom. 110, 73 (2018)

20. Choquet-Bruhat, Y., Chruściel, P.T., Martín-García, J.M.: The cauchy problem on a characteristic cone for the einstein equations in arbitrary dimensions. Ann. Henri Poincaré 12, 419 (2011)

21. Chruściel, P.T., Paetz, T.-T.: The many ways of the characteristic cauchy problem. Class. Quant. Grav. 29, 145006 (2012)

22. Cabet, A., Chruściel, P.T., Wafo, R.T.: On the characteristic initial value problem for nonlinear symmetric hyperbolic systems, including Einstein equations. Diss. Math. 515, 1 (2016)

23. Cagnac, F.: Problème de cauchy sur un conoïde caractéristique. Ann. Fac. Sci. Toulouse $2^{e}$ série 2, 11 (1980)

24. Cagnac, F.: Problème de cauchy sur un conoïde caractéristique pour des equations quasi-lineaires. Ann. Math. Pure Appl. 129, 13 (1981)

25. Lindblad, H., Rodnianski, I.: Global existence for the Einstein vacuum equations in wave coordinates. Commun. Math. Phys. 256, 43 (2005) 
26. Stewart, J.: Advanced General Relativity. Cambridge University Press, Cambridge (1991)

27. Penrose, R., Rindler, W.: Spinors and Space-Time. Two-Spinor Calculus and Relativistic Fields, vol. 1. Cambridge University Press, Cambridge (1984)

28. Valiente Kroon, J.A.: Conformal Methods in General Relativity. Cambridge University Press, Cambridge (2016)

29. Evans, L.C.: Partial Differential Equations. American Mathematical Society, Providence (1998)

Publisher's Note Springer Nature remains neutral with regard to jurisdictional claims in published maps and institutional affiliations. 